\title{
Cembranoids of Soft Corals: Recent Updates and Their Biological Activities
}

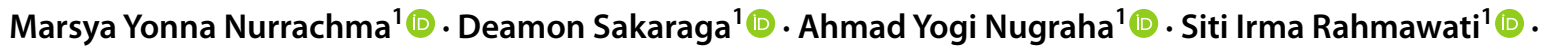

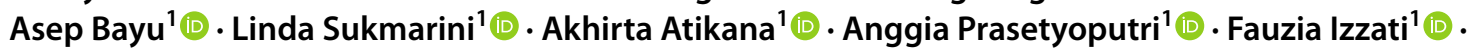 \\ Mega Ferdina Warsito ${ }^{1} \odot \cdot$ Masteria Yunovilsa Putra ${ }^{1}[$
}

Received: 20 January 2021 / Accepted: 6 April 2021 / Published online: 22 April 2021

(c) The Author(s) 2021

\begin{abstract}
Soft corals are well-known as excellent sources of marine-derived natural products. Among them, members of the genera Sarcophyton, Sinularia, and Lobophytum are especially attractive targets for marine natural product research. In this review, we reported the marine-derived natural products called cembranoids isolated from soft corals, including the genera Sarcophyton, Sinularia, and Lobophytum. Here, we reviewed 72 reports published between 2016 and 2020, comprising 360 compounds, of which 260 are new compounds and 100 are previously known compounds with newly recognized activities. The novelty of the organic molecules and their relevant biological activities, delivered by the year of publication, are presented. Among the genera presented in this report, Sarcophyton spp. produce the most cembranoid diterpenes; thus, they are considered as the most important soft corals for marine natural product research. Cembranoids display diverse biological activities, including anti-cancer, anti-bacterial, and anti-inflammatory. As cembranoids have been credited with a broad range of biological activities, they present a huge potential for the development of various drugs with potential health and ecological benefits.
\end{abstract}

Asep Bayu

asepbayu@yahoo.co.id; asep046@ lipi.go.id

Masteria Yunovilsa Putra

mast001@lipi.go.id; masteria.yunovilsa@gmail.com

1 Research Center for Biotechnology, Indonesian Institute

of Sciences (LIPI), Jalan Raya Jakarta-Bogor KM. 46,

Cibinong, Bogor, West Java, Indonesia 


\section{Graphic Abstract}

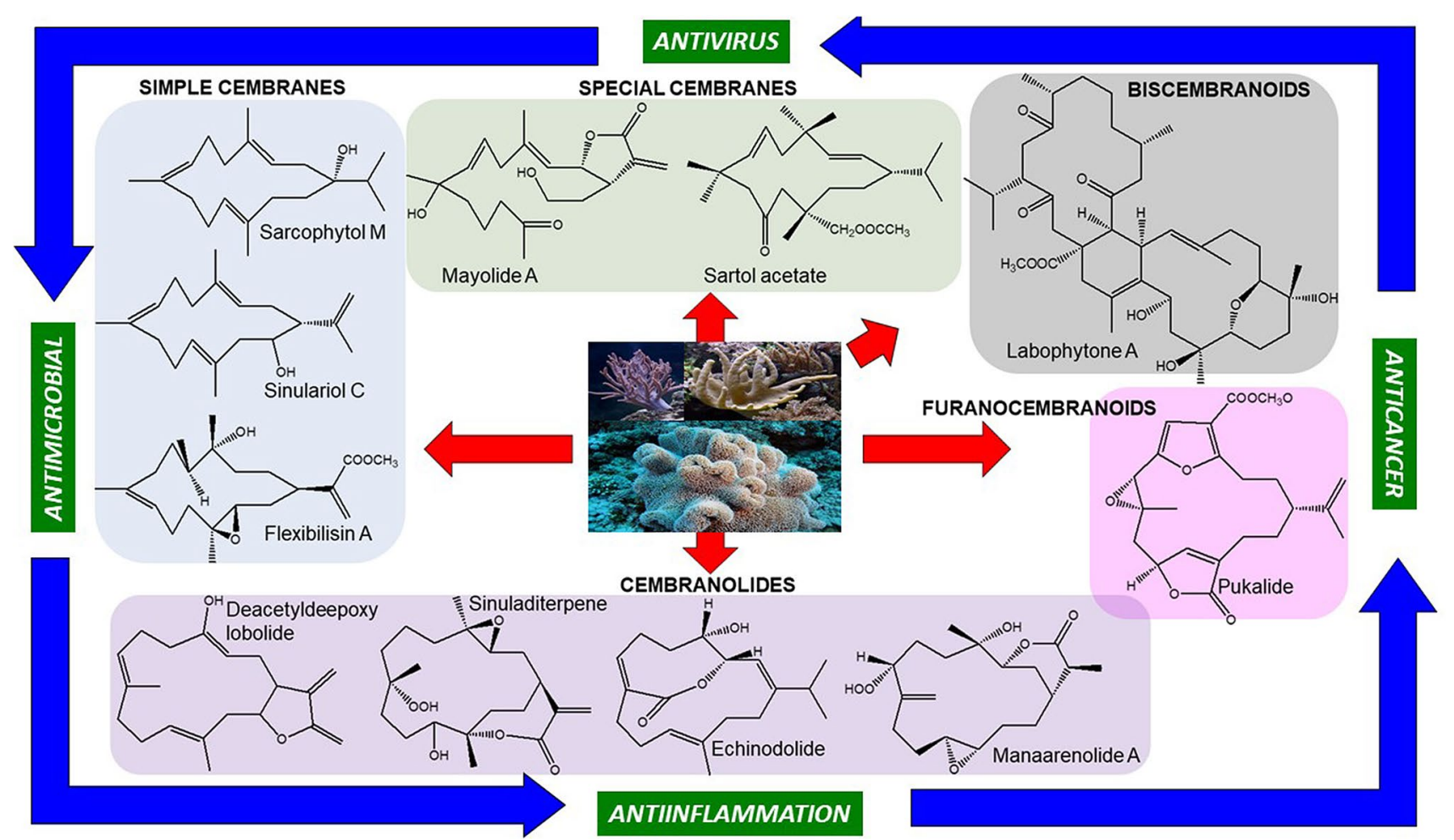

Keywords Cembranoids · Diterpene $\cdot$ Soft corals $\cdot$ Sarcophyton $\cdot$ Sinularia $\cdot$ Lobophytum $\cdot$ Anti-bacterial $\cdot$ Anti-cancer . Anti-inflammatory

\section{Introduction}

The ocean represents the largest habitat on earth, covering over $70 \%$ of the earth surface and harboring a large number of marine organisms whose living environments are quite different from those of their land-based counterparts [1-3]. The extreme ocean conditions, e.g. high pressure, high salinity, hypoxia, and low light levels [4], lead marine organisms to synthesize the highly diverse and unique biological and chemical entities. As a result, the ocean is an important source of natural products with remarkable bioactivities for (novel) drug discovery. Among marine organisms, sessile animals such as soft corals have been shown to have strong chemical defense systems, which are reflected in the almost infinite structural diversity and complexity of their secondary metabolites. Hence, these organisms have long attracted the interest of natural product chemists for drug discovery research and development [5].

Soft corals (phylum, Cnidaria; class, Anthozoa; subclass, Octocorallia; order, Alcyonaceae; family, Alcyoniidae) have been studied as sources of marine-derived natural products since the nineteenth century [6]. They are generally found in Indo Pacific reefs, whereas Gorgonian octocorals dominate the biomass in coral reef environments of the north-western Atlantic Ocean and the Caribbean Sea [7]. The subclass Octocorallia including soft corals, gorgonians, and sea pens, are the most commonly studied corals for drug discovery [8]. The main natural product isolated from soft corals is cembranoids, which act as chemical defense compounds against fish predators. Generally, these metabolites are obtained from the genera Sarcophyton, Sinularia, Lobophytum, Eunicea, and Clavularia [7, 9-11]. Among all, the first three genera attract the most interest in the study of cembranoids [6].

Cembranoids are derived from the cyclization of geranylgeranyl pyrophosphate [12], as shown from the double bonds of the cembrane skeleton having the E geometry observed in geranylgeraniol, diterpene alcohol. Theyare a class of isoprenoid and consist of a fourteen-membered carbocyclic ring with an isopropyl residue at position 1, and three methyl groups at positions 4, 8, and 12 [9, 13, 14]. Cembrane diterpenoids have diverse structural variations with many functional groups (lactone, epoxide, furan, ester, aldehyde, hydroxyl, carboxyl moieties) and cyclizations 
that allow them to be grouped into several families $[15,16]$. According to the review of Yang et al. [15], the cembranetype diterpenoids may be classified as shown in Fig. 1, which are:

(1) Simple cembranes include the isopropyl cembranes, isopropenyl cembranes, and isopropyl/isopropenyl acid cembranes subtypes.

(2) Cembranolides possess a 14-membered carbocyclic nucleus generally fused to a 5-, 6-, 7-, or 8-membered lactone ring. Cembranolides include the subtypes 5-membered lactone, 6-membered lactone, 7-membered lactone, 8-membered lactone.

(3) Furanocembranoids possess a 14-membered carbocyclic nucleus as well as a furan heterocycle. They also have a butenolide moiety involving C-10-C-12 and C-20.

(4) Biscembranoids possess a 14-6-14 membered tricyclic backbone of tetraterpenoids.

(5) Special cembranes include the subtypes secocembranes, 13-membered carbocyclic cembranoids, cembrane glycosides, cembrane africanane, and other cembranes.

This review highlights secondary metabolites isolated from the genera Sarcophyton, Sinularia, Lobophytum and their biological activities reported in the literature between 2016 to mid-2020. The literatures were collected from different online databases, including Pubmed and Google Scholar, presenting the research progress on secondary metabolites isolated from soft corals within the last five years. This review summarizes the potential application of biomolecules (360 compounds) isolated from these three genera, covering the chemistry as well as the biological activity of their secondary metabolites, with special reference to cembranoids.

\section{Cembranoids}

\subsection{Cembranoids Reported from Genus Sarcophyton}

A total of 169 cembranoid compounds were isolated from Sarcophyton collected from various geographical areas (Table 1). Out of those, 128 were new compounds and 41 were previously known compounds with newly discovered activities. Eleven of the new compounds were newly discovered and have not been thoroughly tested for their biological activities.

Cembrane diterpenes have been isolated in a number of different locations. Fresh soft coral Sarcophyton sp. from Karah Island, Terengganu, West Malaysia yielded a new

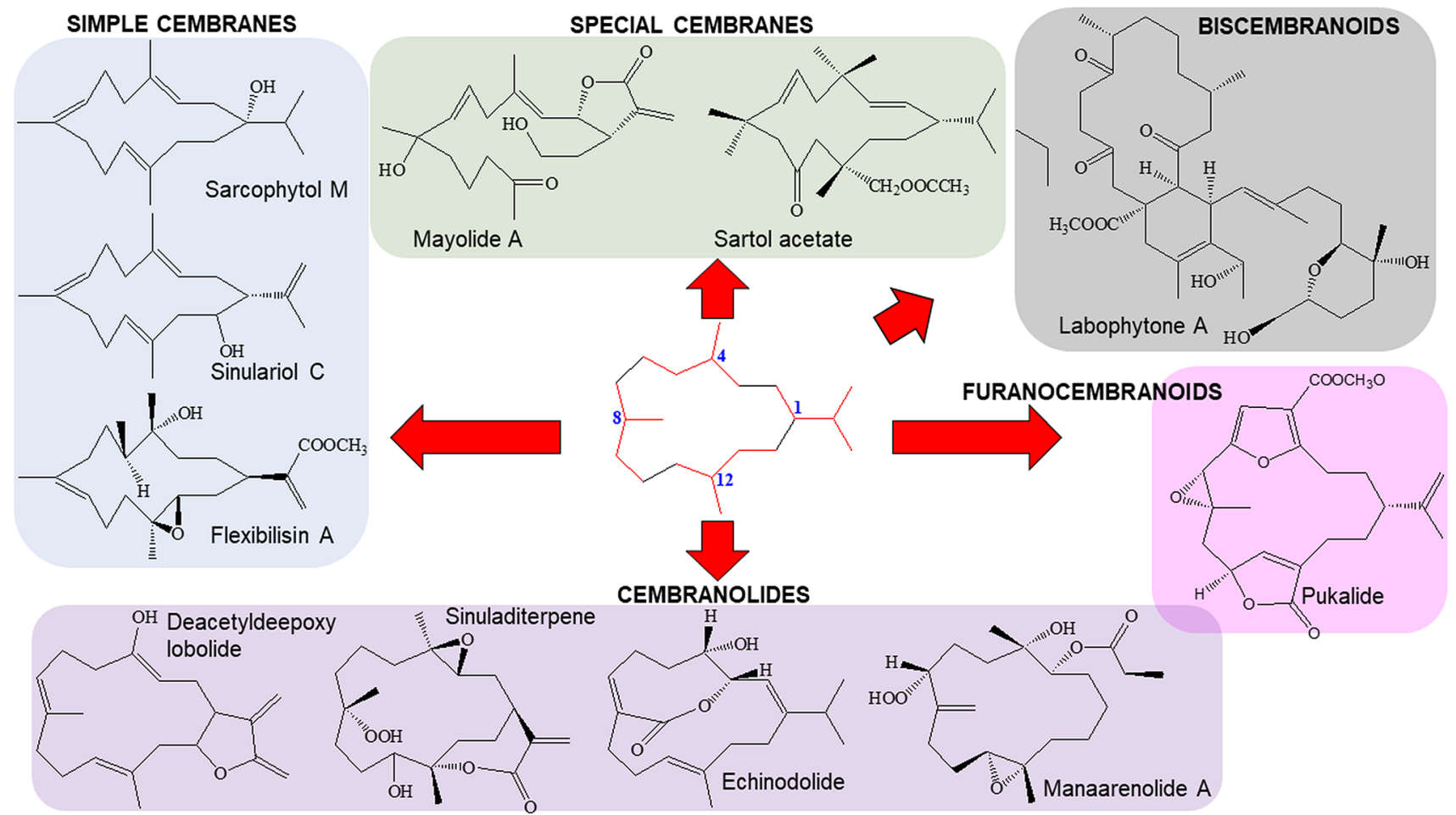

Fig. 1 Chemical structures of chembranoid molecules. The isoprene unit of the basic carbon skeleton of cembranoids is bonded head-to-tail (red colors) 


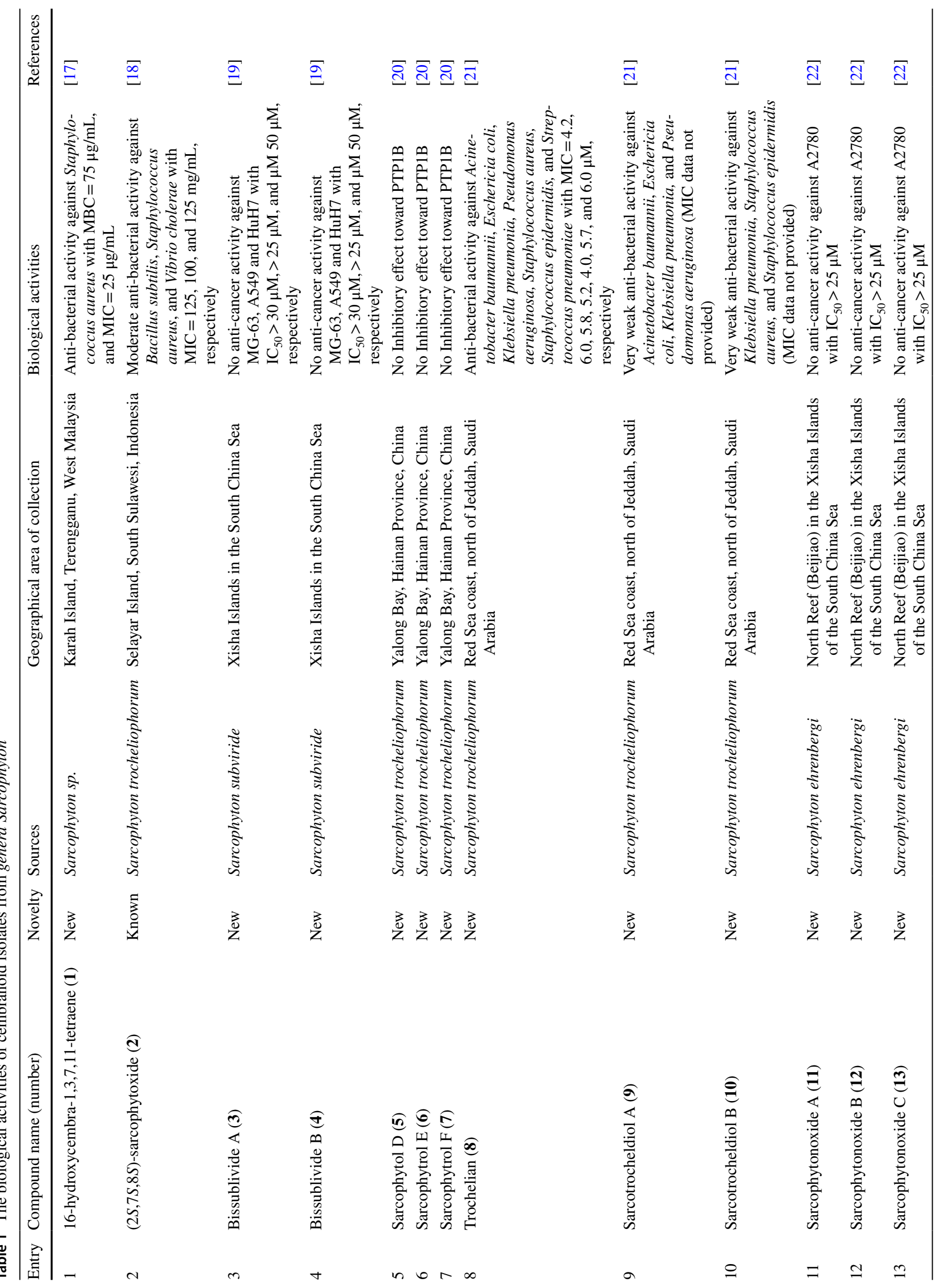




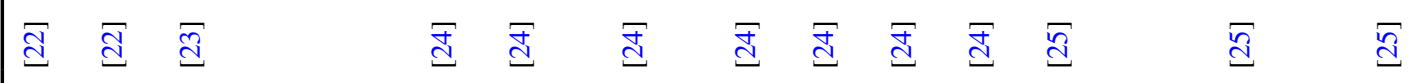

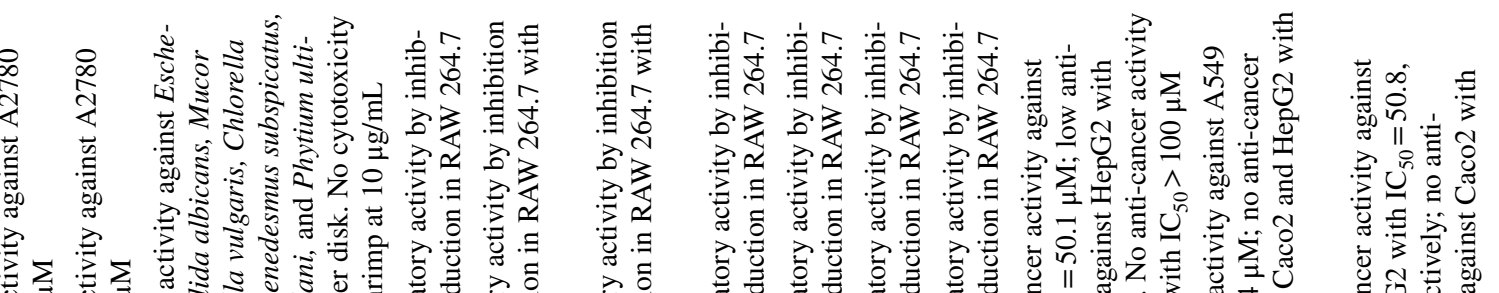

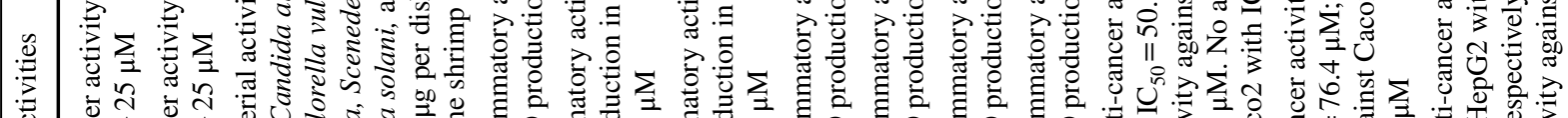

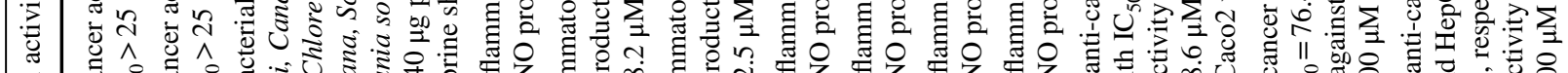

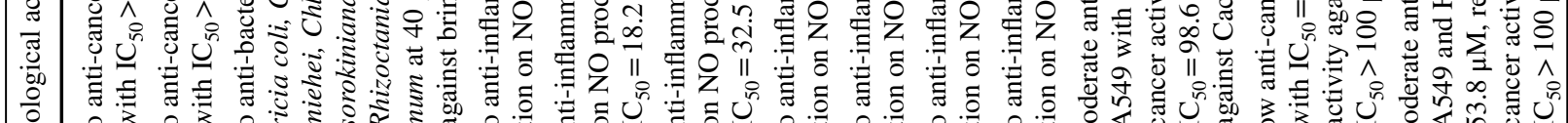

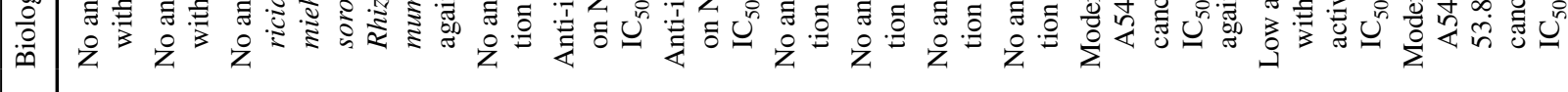
咅

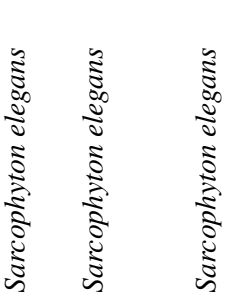

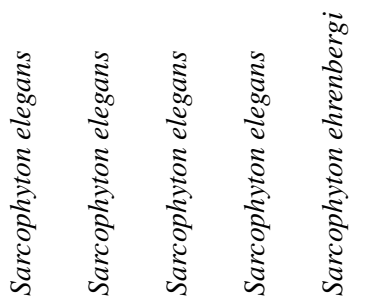

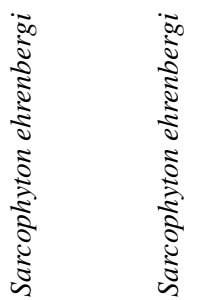

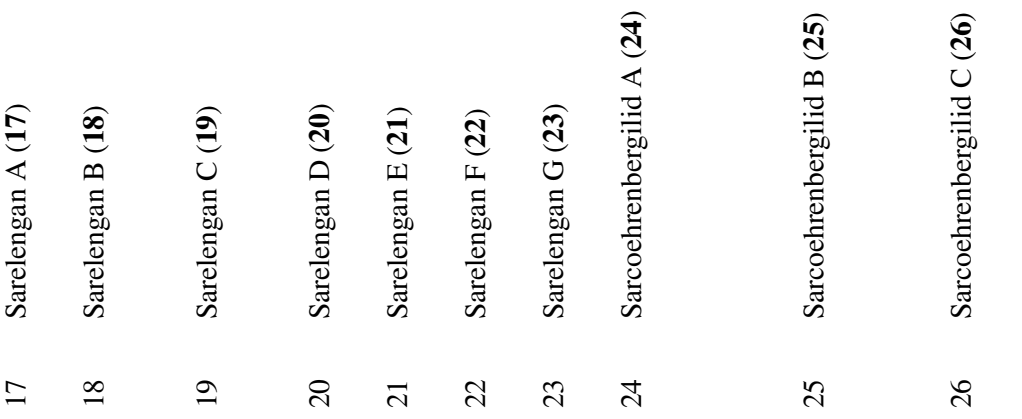




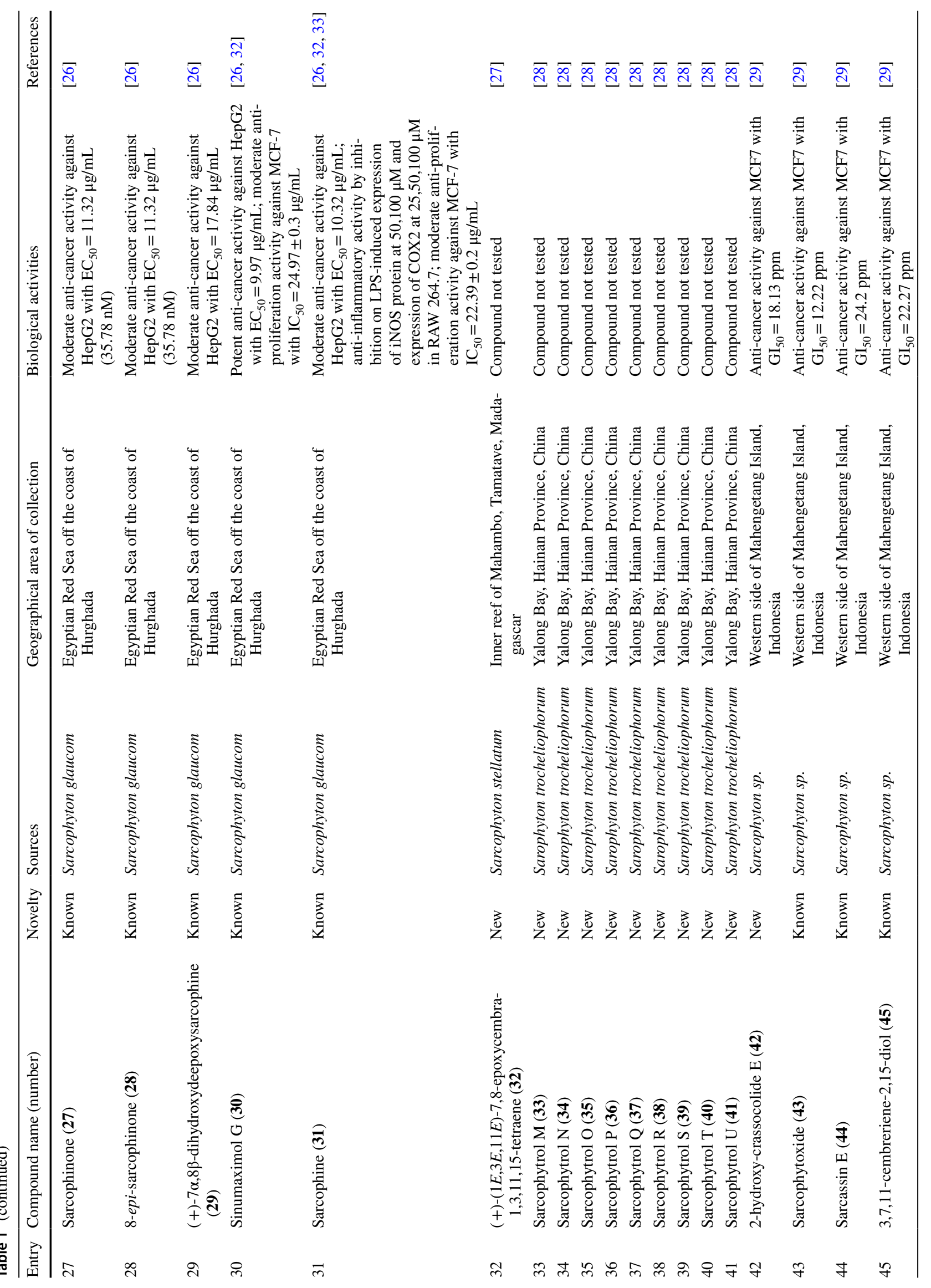




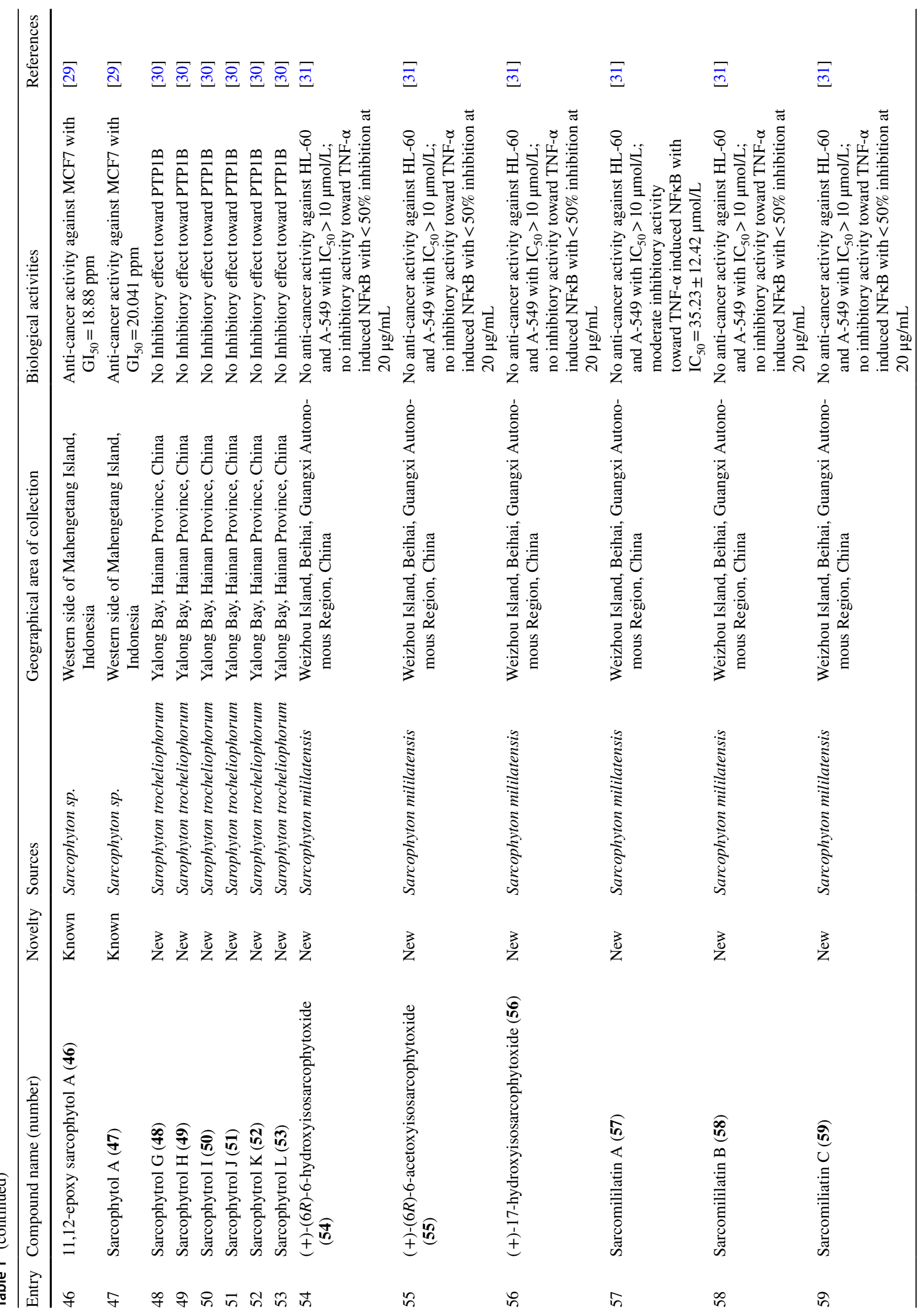




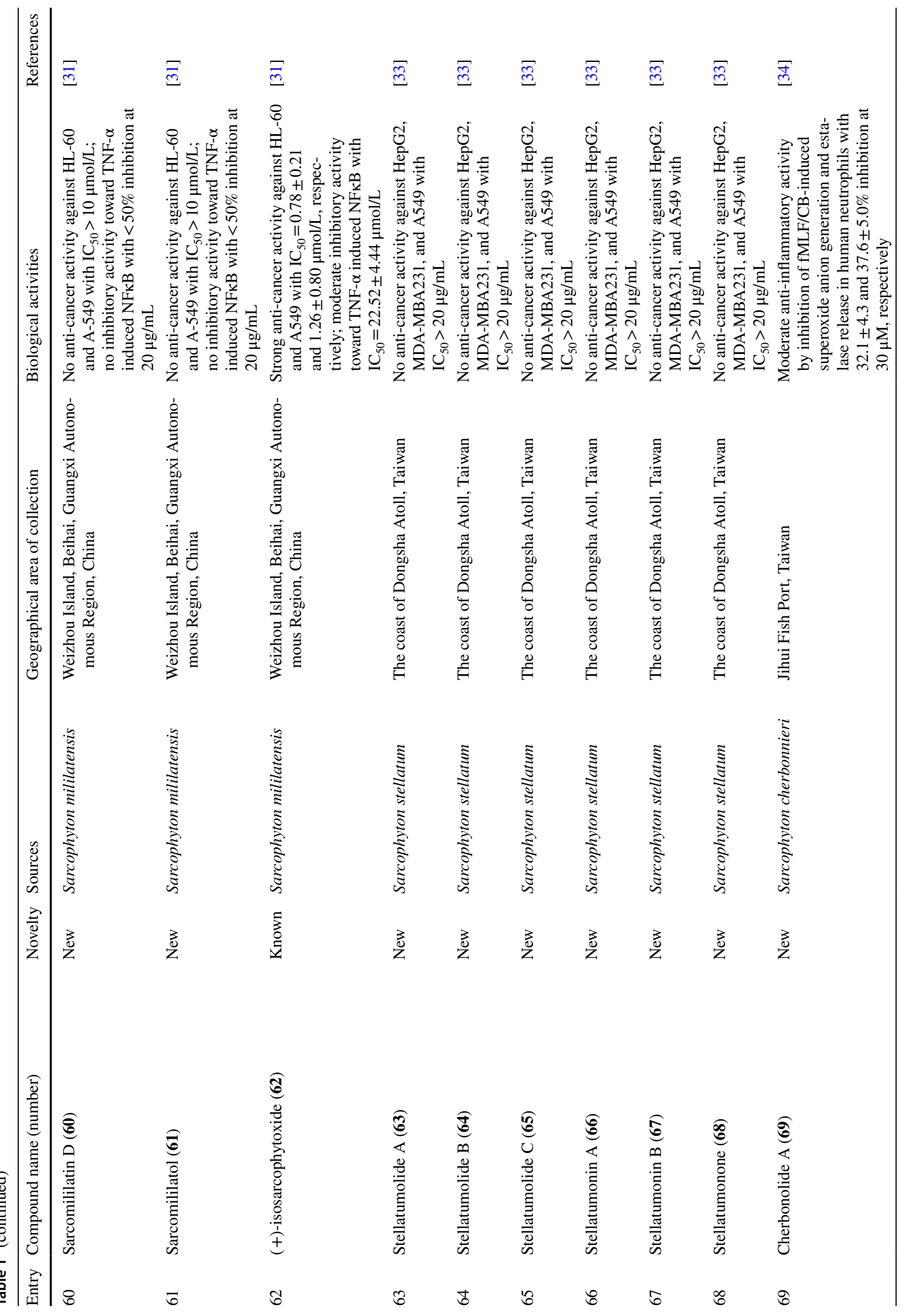




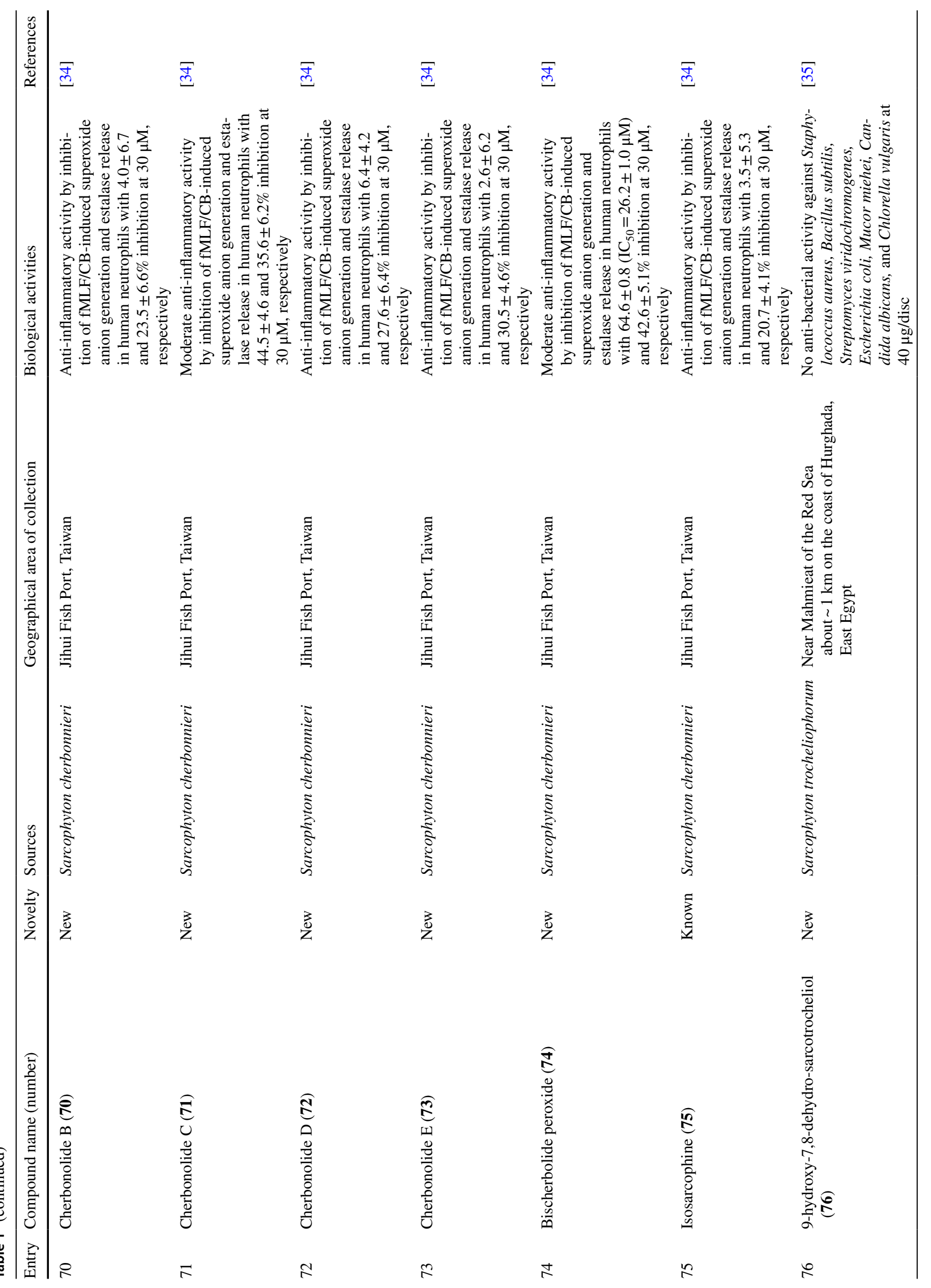




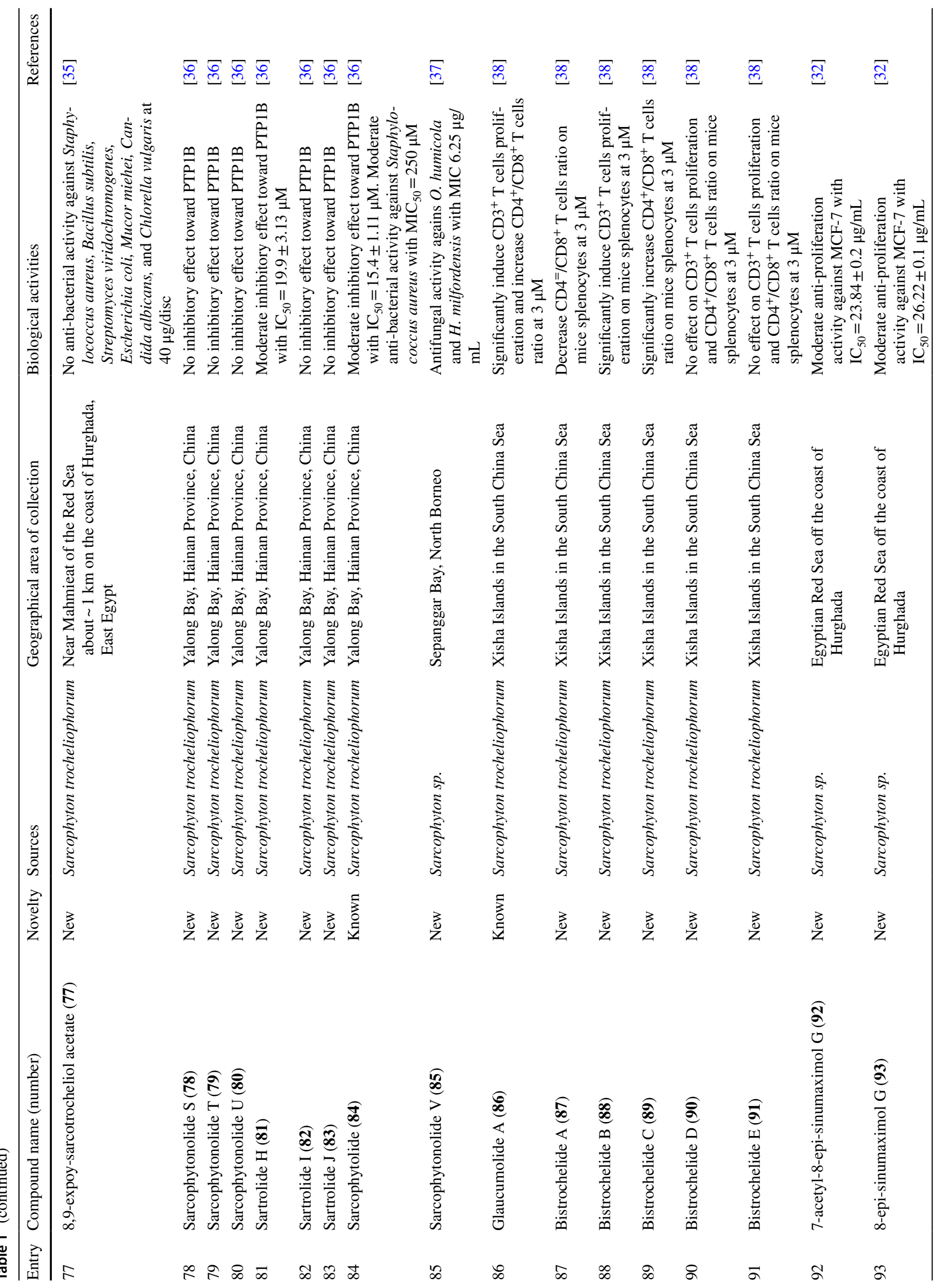




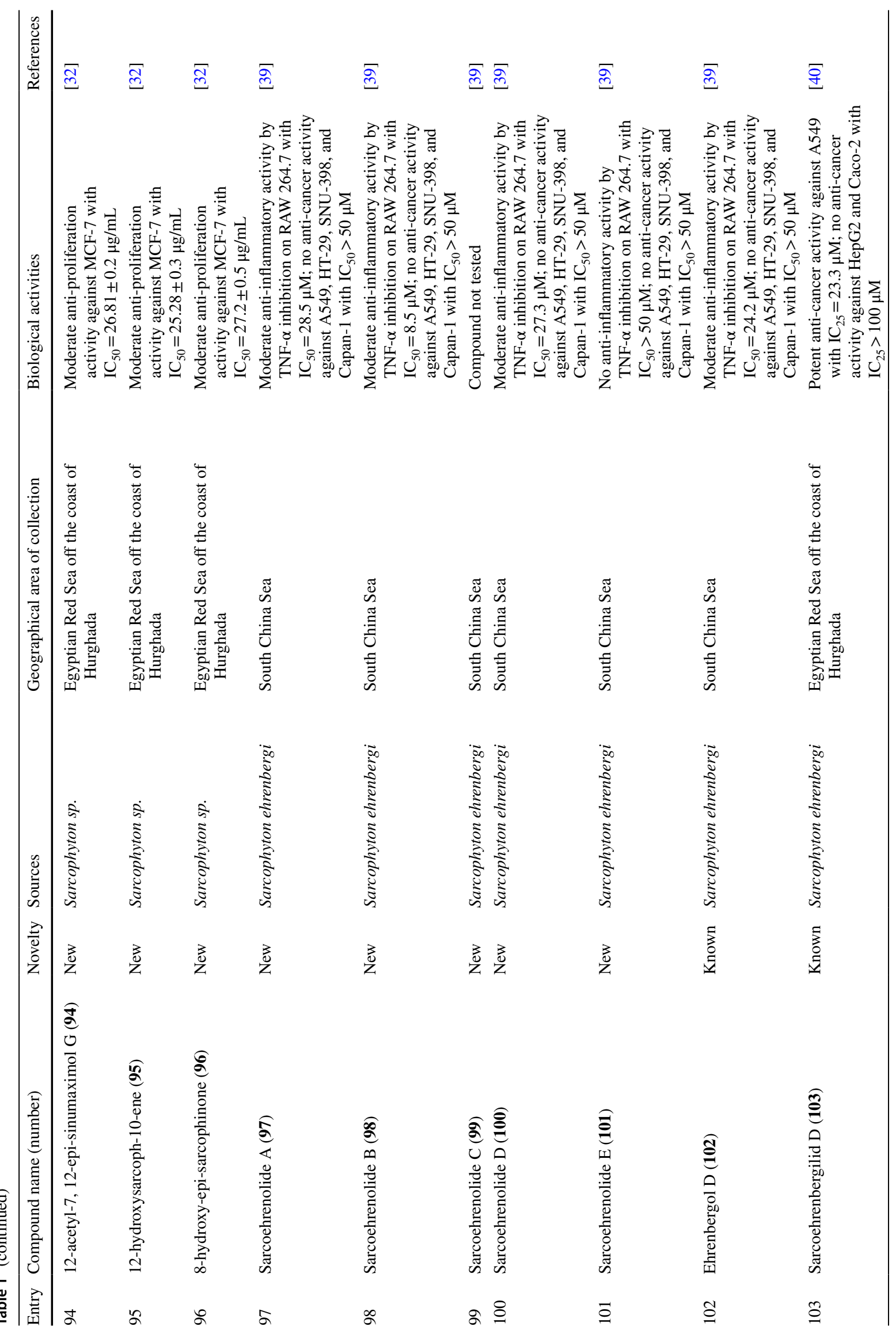




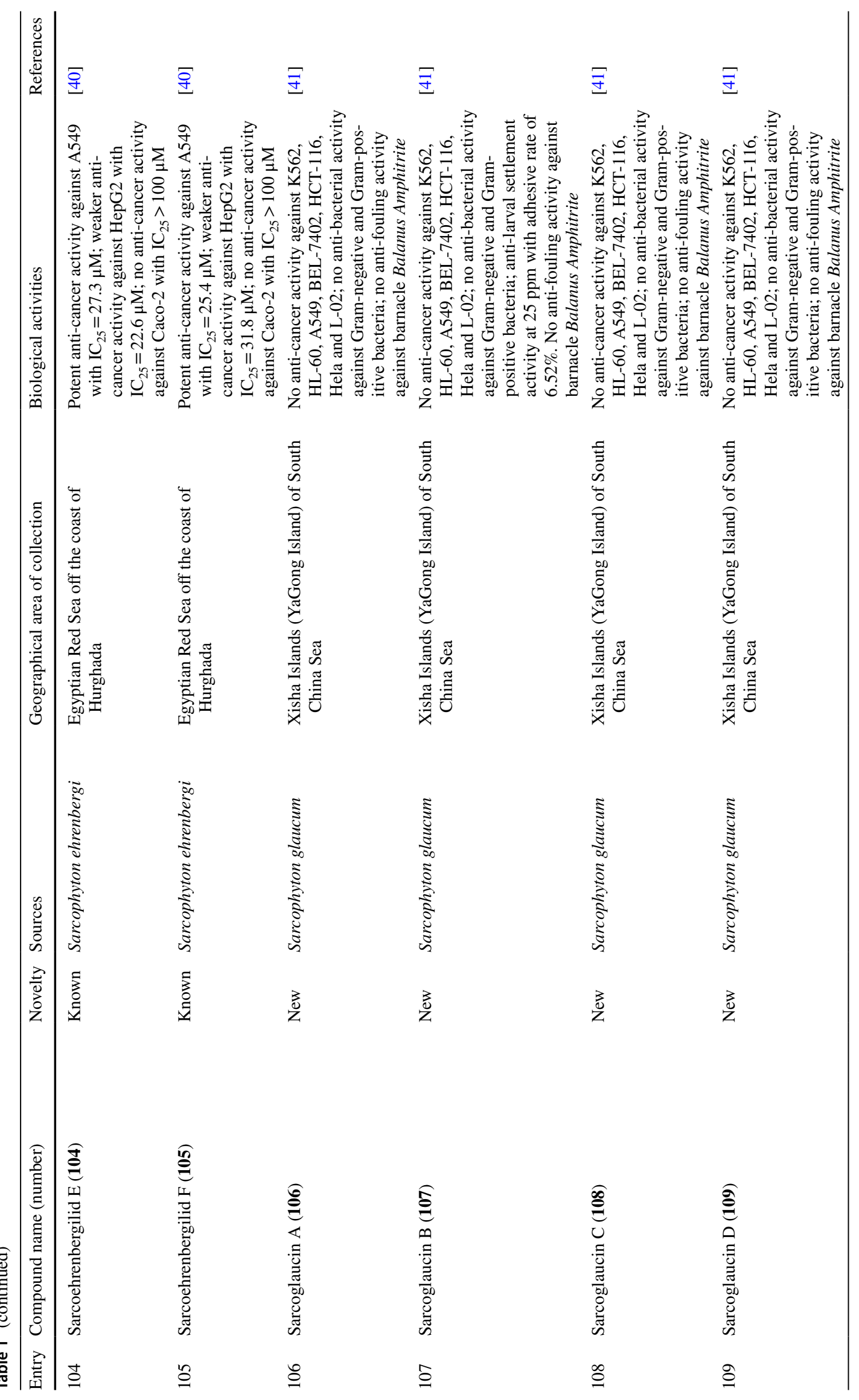




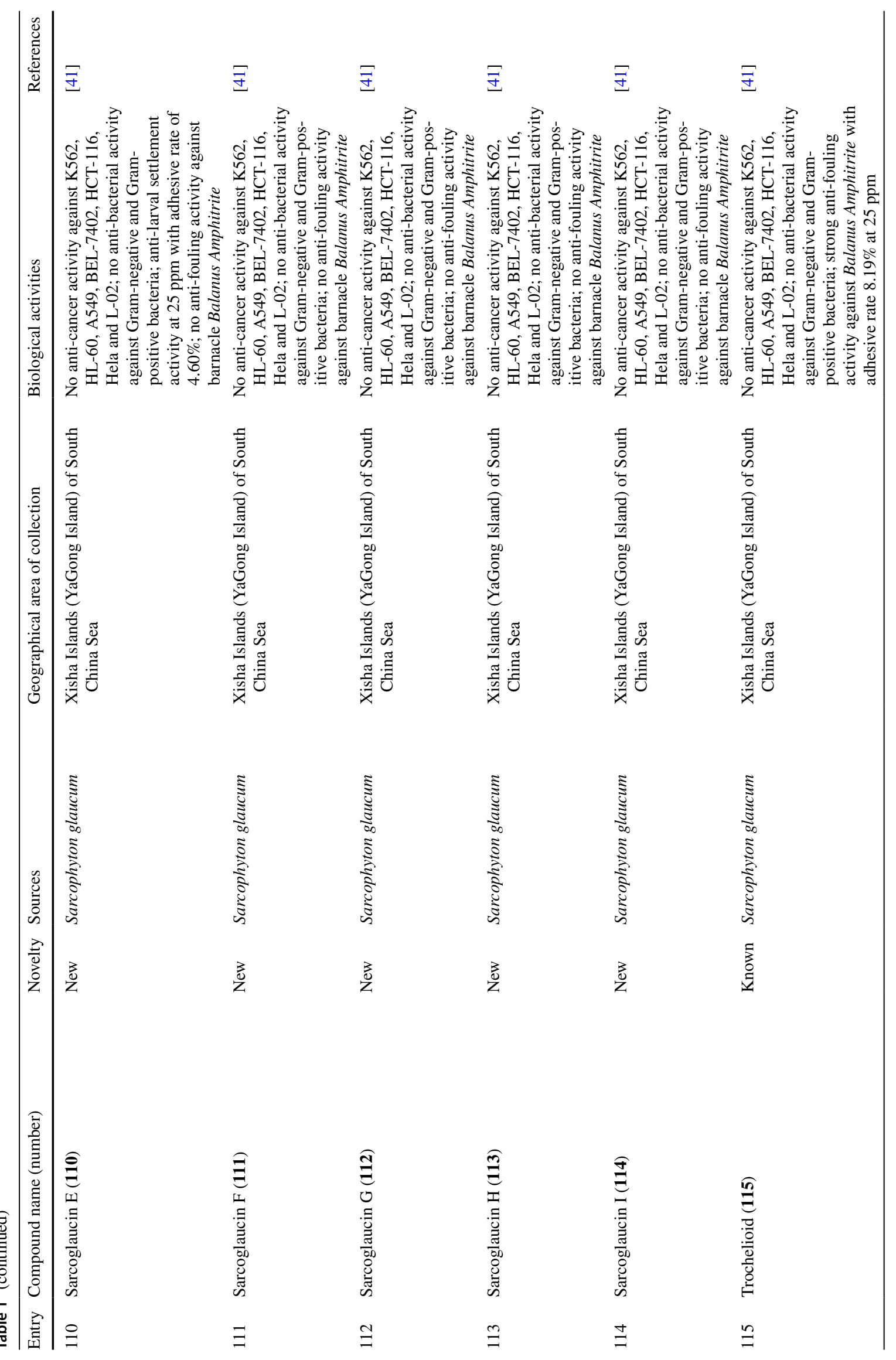




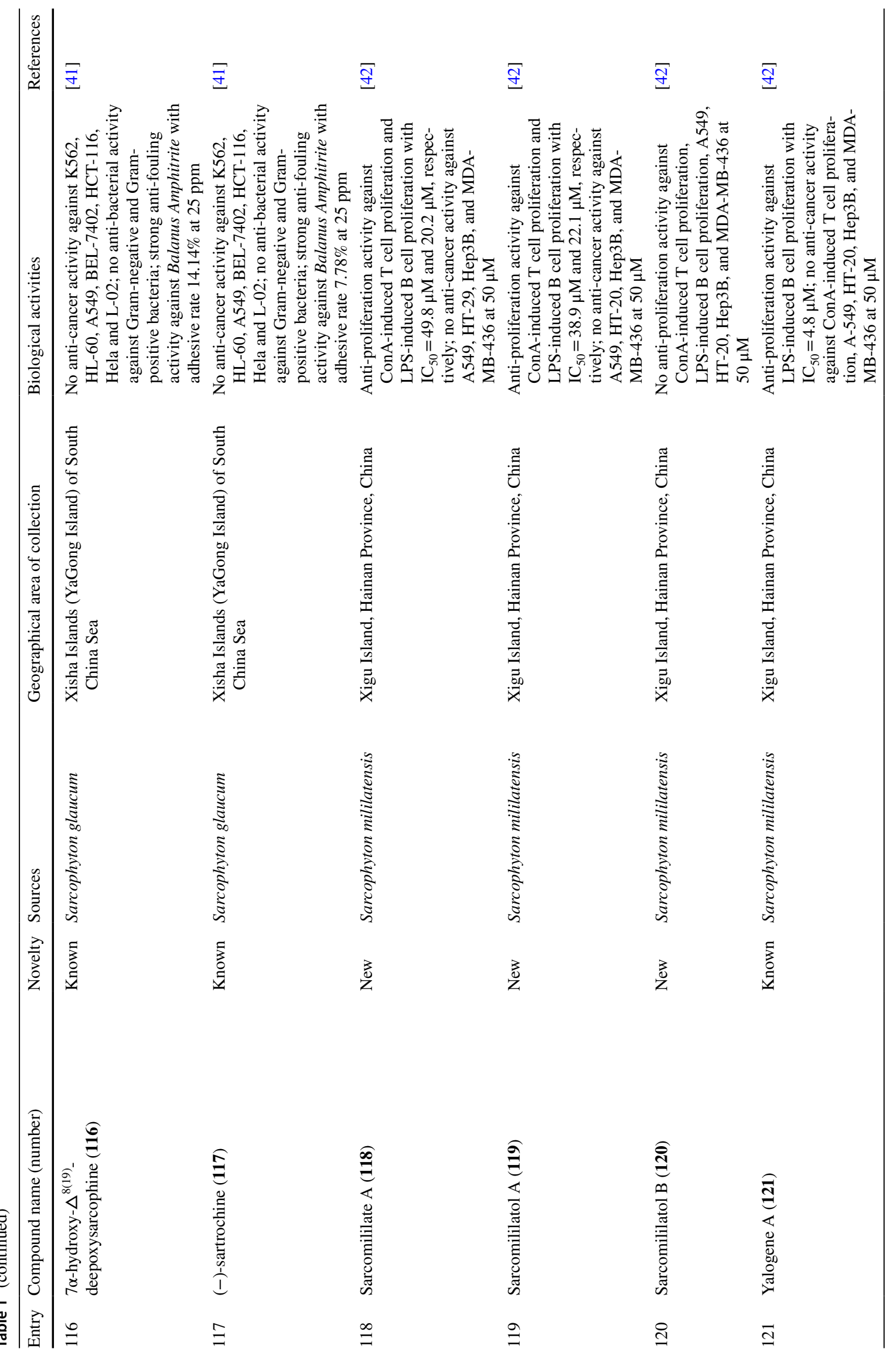




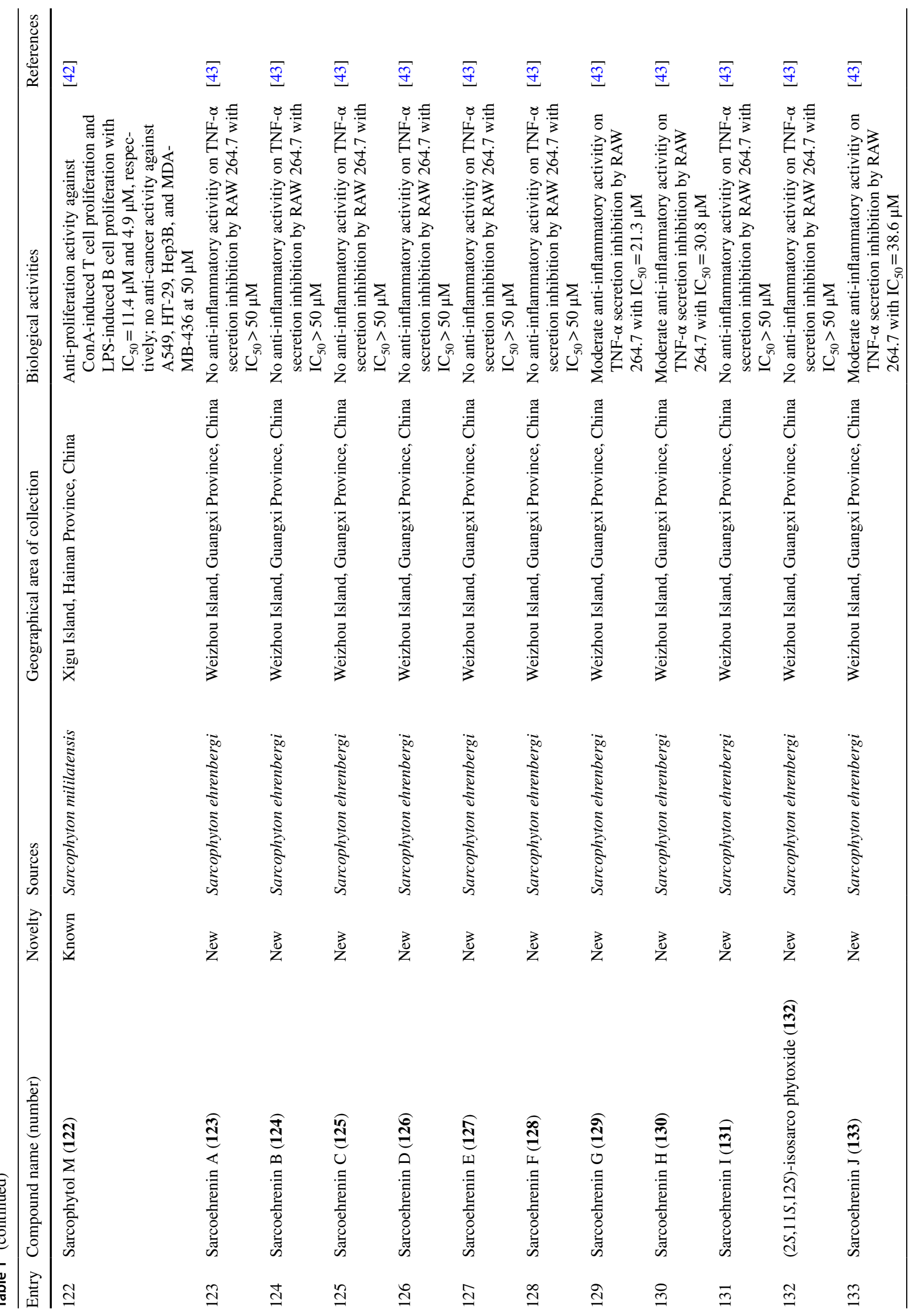




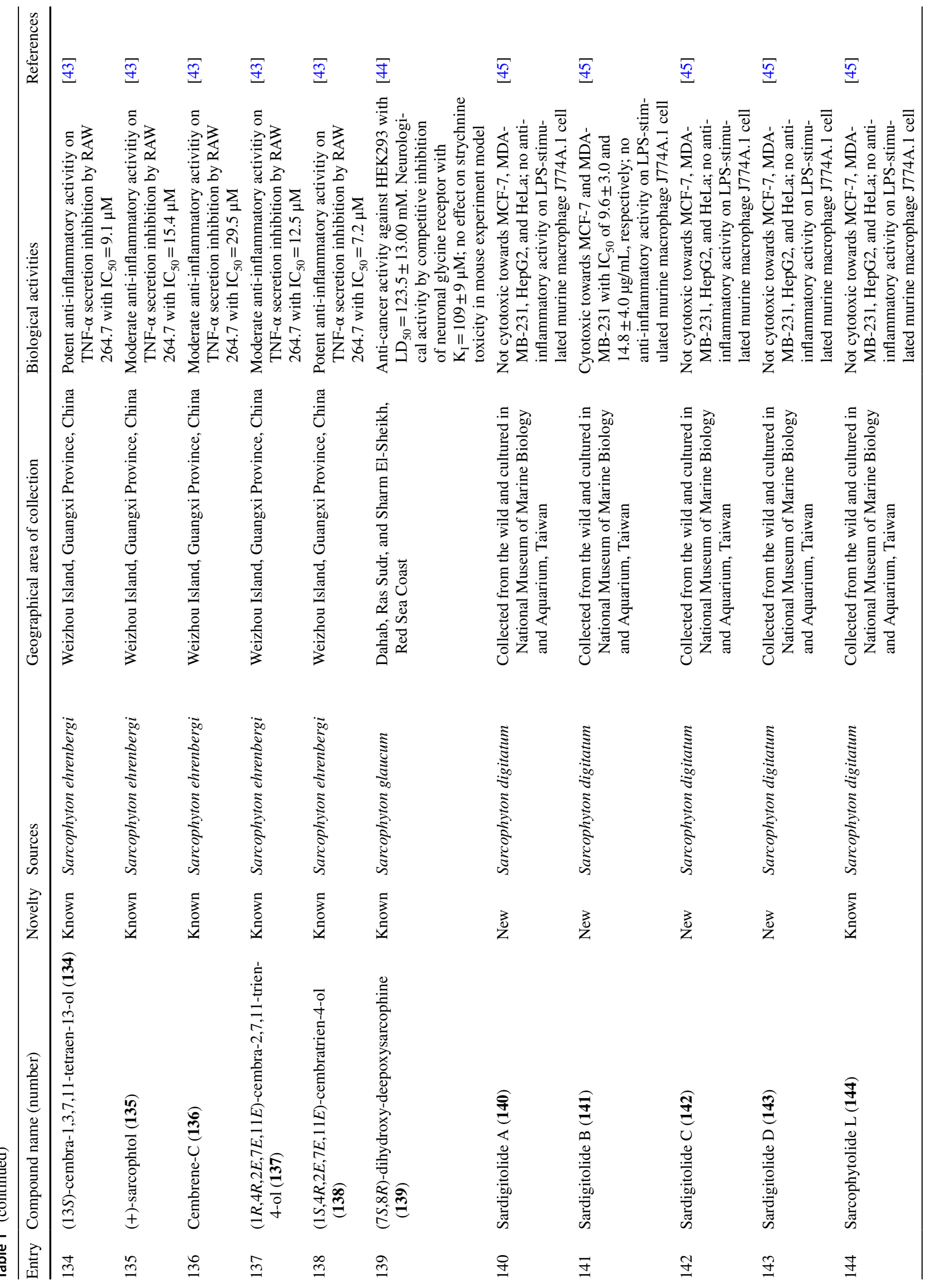




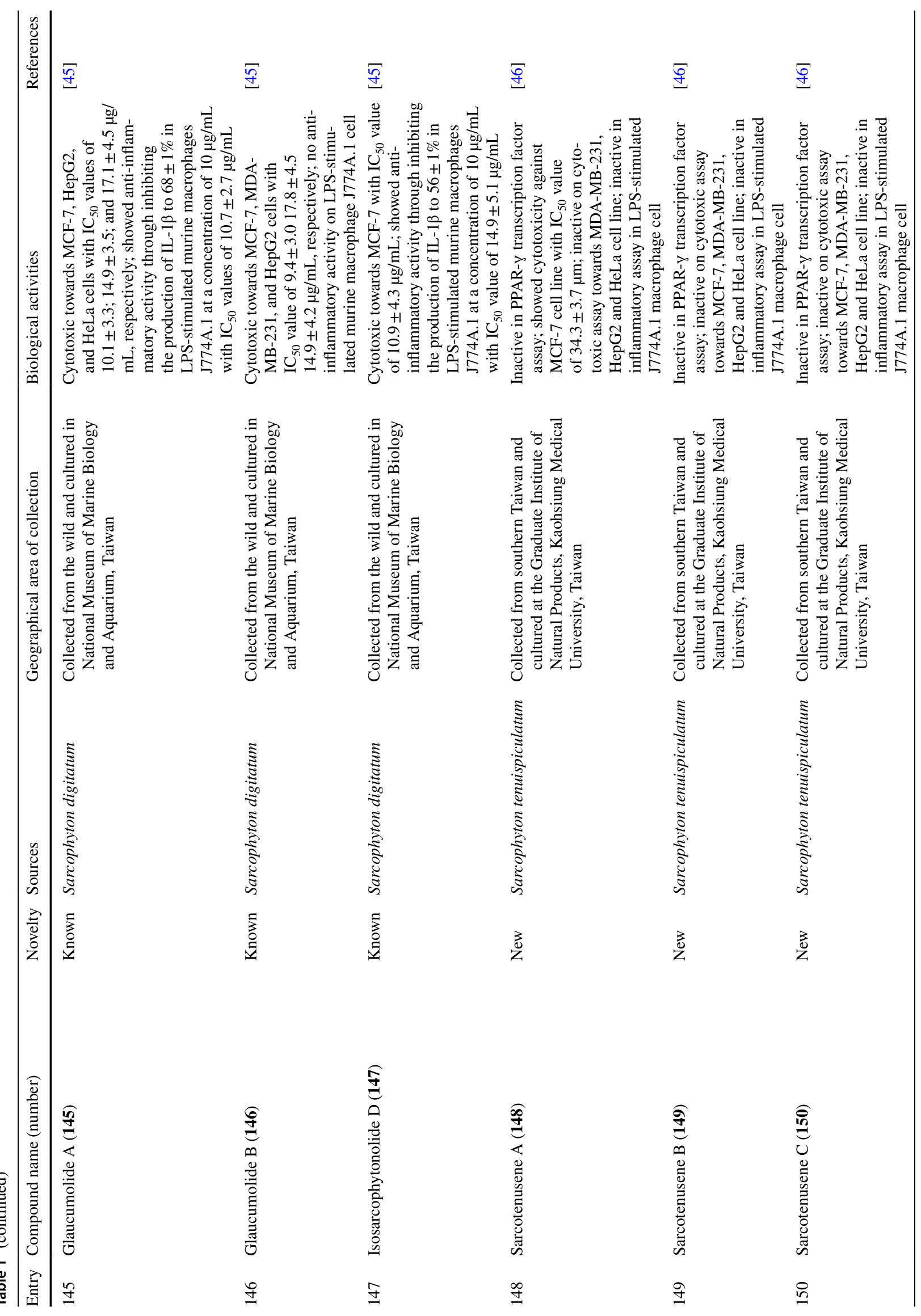




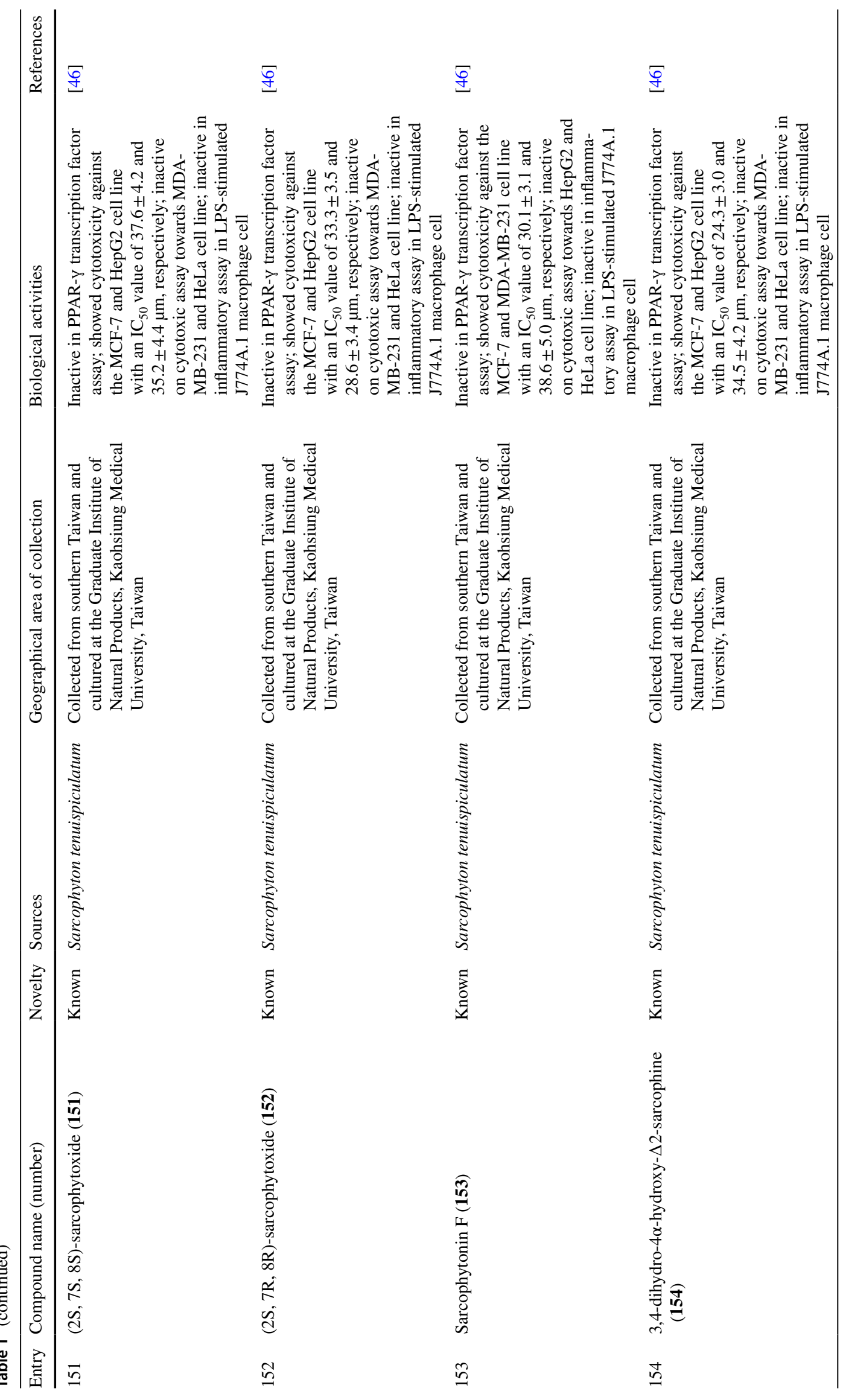




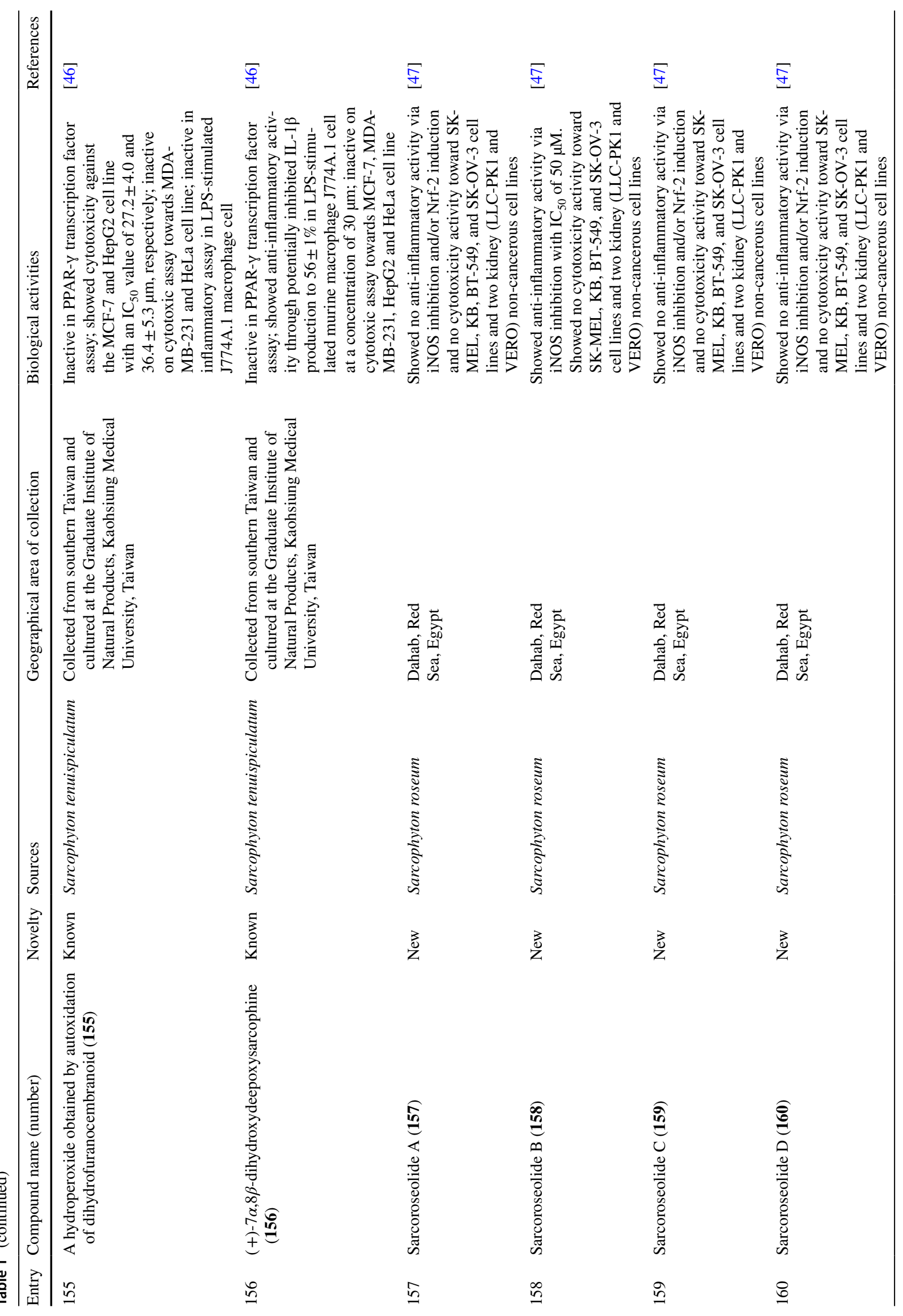




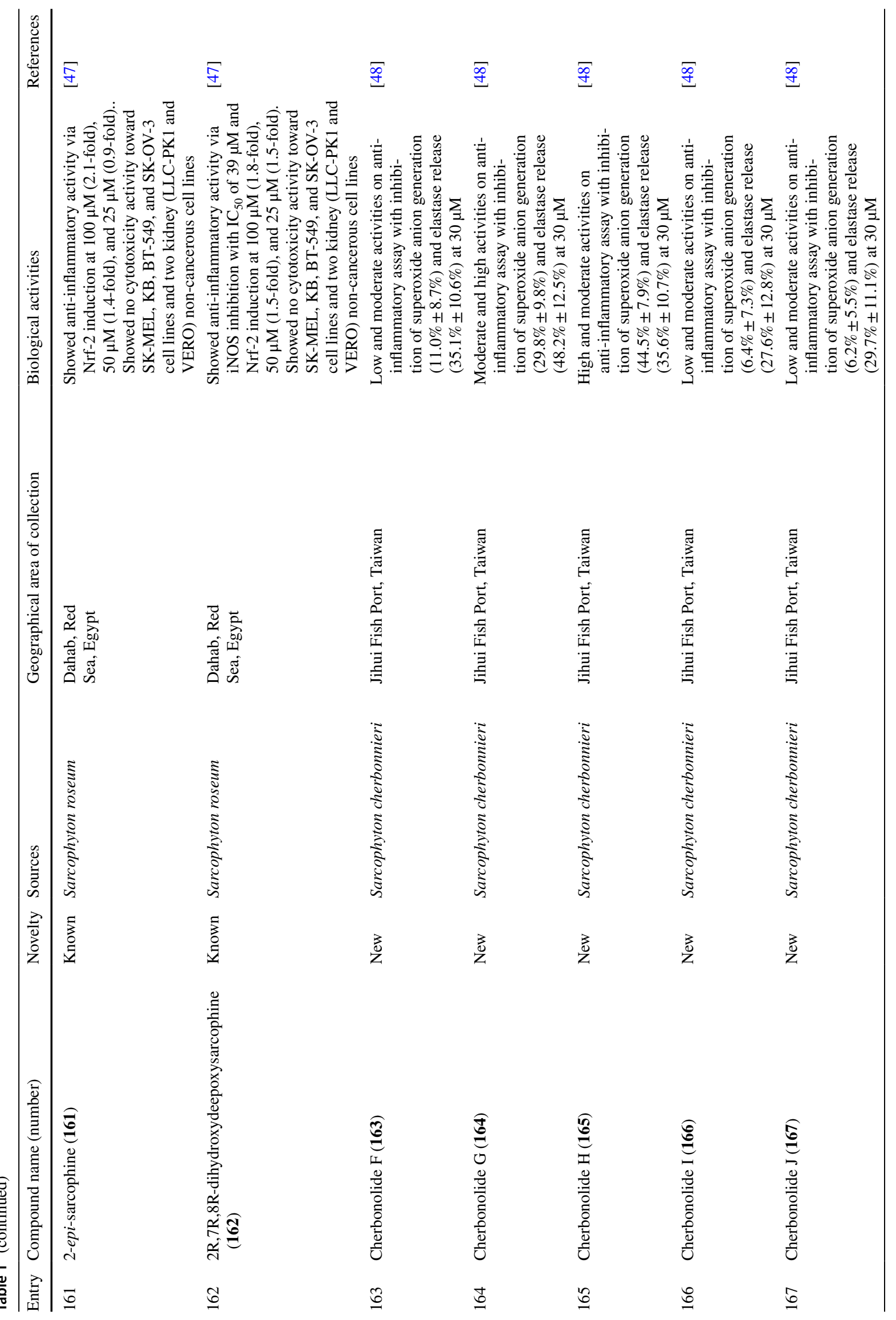




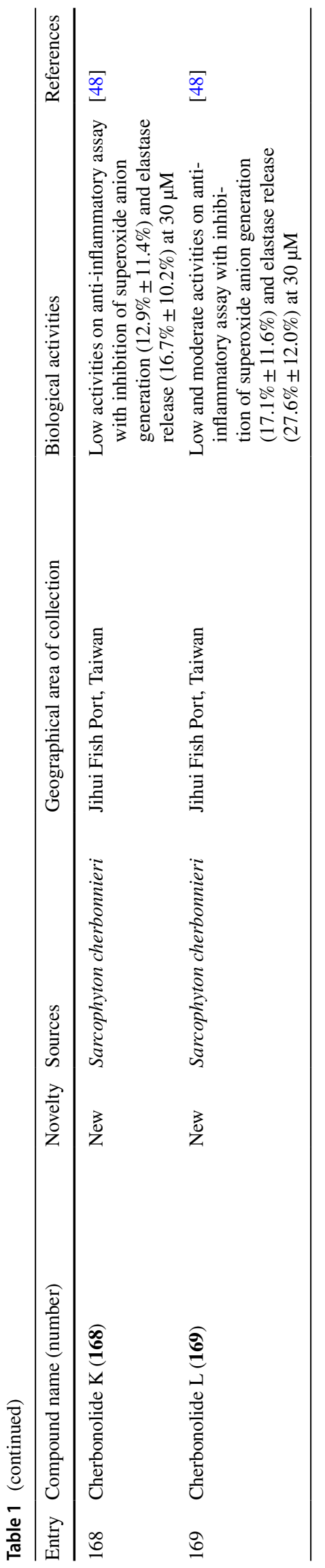

cembrane diterpene, 16-hydroxycembra-1,3,7,11-tetraene 1 (Fig. 2) [17]. The compound is a colorless oil, [ $\alpha] D$ 25: -9.3 (c $0.18, \mathrm{CHCl} 3$ ) with the molecular formula of $\mathrm{C}_{20} \mathrm{H}_{32} \mathrm{O}$ (HR-MS m/z 289.2486 [M+H]+, calcd. 289.2526). A known compound cembranoid diterpene compound, sarcophytoxide 2, was isolated as yellow crystalline needles ( $\sim 0.5 \%$ yield) from the $\mathrm{n}$-hexane fraction of Sarcophyton trocheliophorum collected in Selayar Island, South Sulawesi, Indonesia (Fig. 3). This compound has a molecular formula of $\mathrm{C}_{20} \mathrm{H}_{30} \mathrm{O}_{2}\left(\mathrm{~m} / \mathrm{z} 325[\mathrm{M}+\mathrm{Na}]^{+}\right.$, ESI-MS positive ion) and been tested for its new anti-microbial activity (Table 1, entry 2) [18]. Two new biscembranoid-like compounds, bissubvilides A-B 3-4 were isolated from Sacrophyton subviride in Xisha Islands, South China Sea. These compounds have been tested for their anti-cancer activity but showed no activity (Table 1, entries 3,4) [19]. S. trocheliophorum from Yalong Bay, China, yielded three new highly oxidative cembranoids sarcophytols D-F 5-7. Unfortunately, none of them showed activities on protein tyrosine phosphatase 1B (PTP1B) inhibitory effect (Table 1, entries 5-7) [20]. Another study isolated a new tetracyclic biscembrane hydrocarbon, trocheliane $8\left(\mathrm{C}_{40} \mathrm{H}_{58}\right)$, along with two new cembranoid diterpenes, sarcotrocheldiols A-B 9-10 $\left(\mathrm{C}_{20} \mathrm{H}_{34} \mathrm{O}_{3}\right)$, from the same species in Red Sea coast, Saudi Arabia [21]. These cembranoids were isolated as gummy materials with $\mathrm{m} / \mathrm{z}$ of $538.4528\left(\mathrm{M}^{+}\right.$, HREIMS) and $322.2500\left(\mathrm{M}^{+}\right.$, HREIMS), respectively.

Five new compounds, sarcophytonoxides A-E 11-15 were isolated from Sarcophyton ehrenbergi in North Reed (Beijiao) in the Xisha Islands, South China Sea (Fig. 4). HRESIMS analysis revealed sarcophytonoxides $\mathrm{A}, \mathrm{C}$ and $\mathrm{E}$ are isomers with the molecular formula of $\mathrm{C}_{22} \mathrm{H}_{32} \mathrm{O}_{4}$. Meanwhile, sarcophytonoxides $\mathrm{B}$ and $\mathrm{D}$ have molecular formula of $\mathrm{C}_{22} \mathrm{H}_{32} \mathrm{O}_{5}$ and $\mathrm{C}_{20} \mathrm{H}_{30} \mathrm{O}_{3}$, respectively. However, these compounds have been tested for anti-cancer activity against human ovarian cancer cell line A2780, however, they showed no effect (Table 1, entries 11-15) [22]. A new pyrane-based cembranoid diterpene, 9-hydroxy-10,11-dehydro-sarcotrocheliol 16, was isolated from S. trocheliophorum. However, this compound showed no anti-bacterial activity against multiple microorganisms (Table 1, entry 16) [23]. A study isolated two novel biscembranoids, sarelengans A-B 17-18, along with five new cembranoids, sarelengans C-G 19-23 from Sarcophyton elegans in Xisha Islands, South China Sea with only 18 and 19 exhibited anti-inflammatory activity (Table 1, entries 17-23) [24]. S. ehrenbergi from the Egyptian Red Sea off the coast of Hurghada yielded three novel cembrene diterpenoids sarcoehrenbergilids A-C 24-26 [25]. Sarcoehrenbergilids A was found as a white crystal with a molecular formula of $\mathrm{C}_{21} \mathrm{H}_{32} \mathrm{O}_{5}\left(\mathrm{~m} / \mathrm{z}\right.$ at $[\mathrm{M}+\mathrm{Na}]^{+}$of 387.2142) while Sarcoehrenbergilids B and C were isomers observed as a white powder with a molecular formula of $\mathrm{C}_{20} \mathrm{H}_{30} \mathrm{O}_{5}\left(\mathrm{~m} / \mathrm{z}\right.$ at $[\mathrm{M}+\mathrm{Na}]^{+}$of 373.1986$)$. Another species 
<smiles>C/C(=C/C=C(\C)CC/C=C\CC/C=C(\C)I)C(C)CO</smiles>

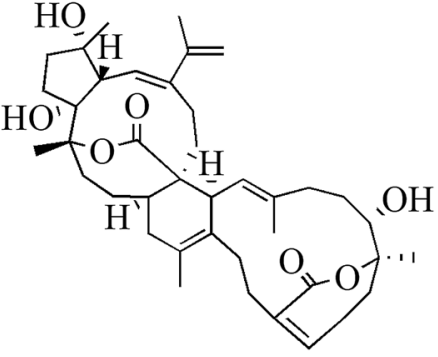

3,4<smiles>C=C1OC(CC2(C)OC2CC2CCC(C)=CC(O)C2C)C(=C)C1=C</smiles>

42<smiles>C/C=C\CC/C(C)=C/CCC1OC1(C)CC(O)/C(=C\C=C(\C)CC)C(C)C</smiles>

43

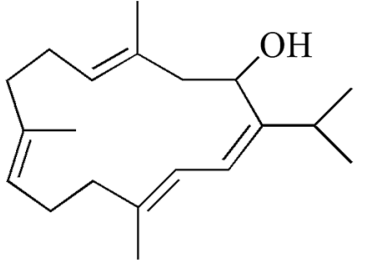

44<smiles>C/C=C(\C)CC/C=C(\C)CCC(C(O)C/C=C(\C)CC/C=C(/C)CC)C(C)(C)O</smiles>

46<smiles>CC1=C(C)C(/C=C(\C)CCC2OC2(C)CC/C=C(\C)CCC2=C(C)OC2)OC1</smiles>

45<smiles>COC(=O)/C(=C/C=C1/CCCC2CCCC(C)(OC2=O)C(=O)CC1)C(C)C</smiles>

47<smiles>C/C1=C\CC[C@@H](C)CC(=O)CC2=C[C@H](OC1=O)[C@@H](C(=O)CC(C)C)C(=O)O2</smiles>

85<smiles>CC1=C2CC[C@@](C)(O)/C=C\C[C@](C)(O)[C@@H](O)[C@H](O)CC/C(C)=C/[C@@H]2OC1=O</smiles>

93<smiles>CC(=O)O[C@@]1(C)/C=C\C[C@@](C)(O)[C@@H](O)CCC1=C(C)C/C=C(\C)C[C@@H]1OC(=O)C(C)=C1O</smiles><smiles></smiles>

95<smiles>CC1=C2CC/C(C)=C/C[C@@](C)(O)C(O)(O)C(=O)CC/C(C)=C/[C@@H]2C(C)=C(O)O1</smiles>

96<smiles></smiles>

92

Fig. 2 Cembranoids isolated from Sarchophyton sp. (1, 42-47, 85, 92-96) and Sarcophyton subviride $(\mathbf{3 , 4})$

Sarcophyton glaucom from the same area was reported to yields five new diterpenes, sarcophinone 27, 8-epi-sarcophinone $\mathbf{2 8}$, (+)-7 $\alpha, 8 \beta$-dihydroxydeepoxysarcophine $\mathbf{2 9}$, sinumaximol G 30, and sarcophine 31 [26]. Several new cembranoids, (+)-(1E,3E,11E)-7,8-epoxycembra-1,3,11,15-tetraene
32 from Sarcophyton stellatum in Inner reef of Mahambo, Tamatave, Madagascar [27], and sarcophytrols M-U 33-41 from S. trocheliophorum in Yalong Bay, Hainan Province, China [28], was discovered but their activities have not been tested (Table 1, entries 32-41). 


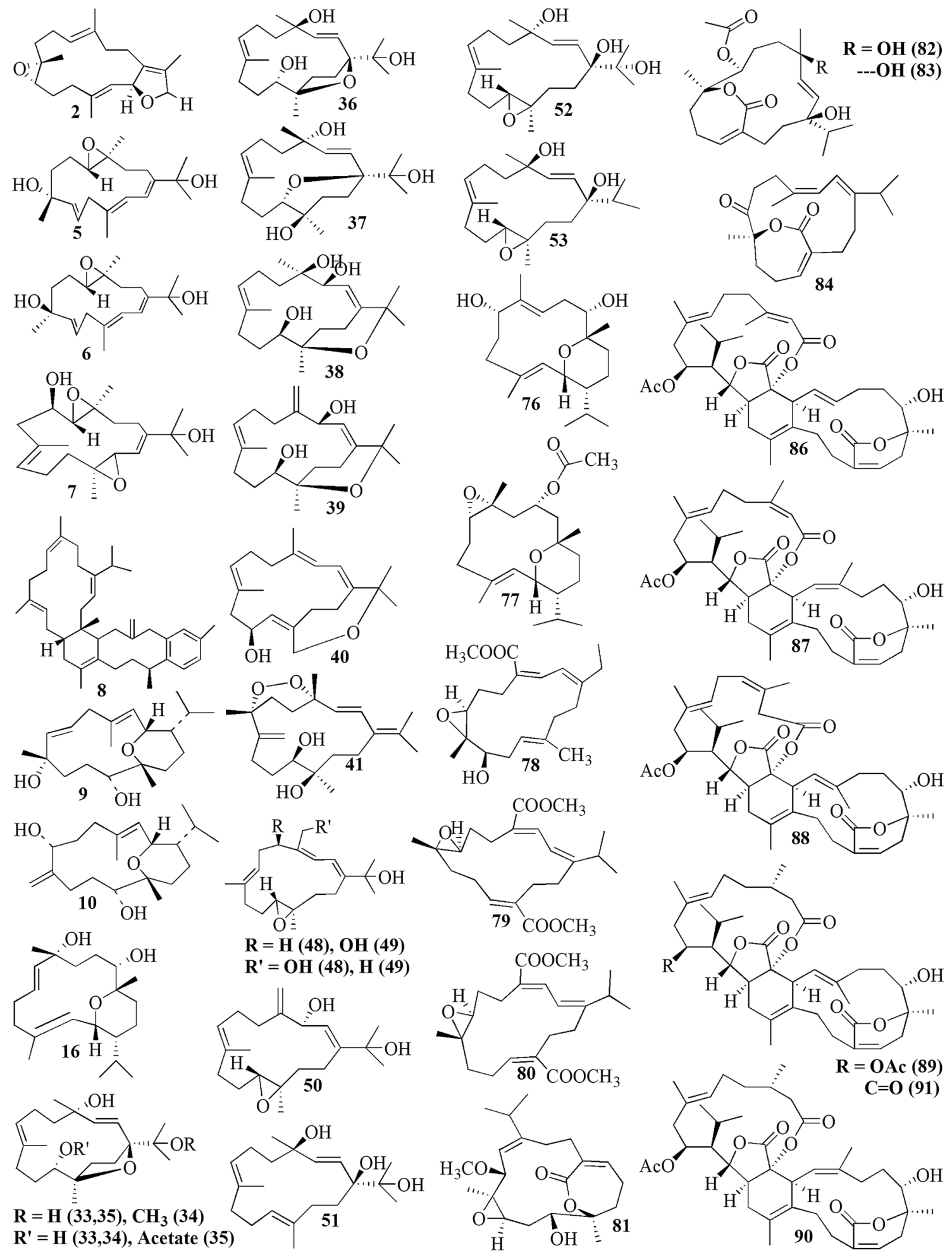

Fig. 3 Cembranoids isolated from Sarchophyton trocheliophorum

A study reported a new cembranoid, 2-hydroxy-crassocolide E 42, and five known cembranoids, sarcophytoxide 43, sarcassin E 44, 3,7,11-cembreriene-2,15-diol 45, 11,12-epoxy sarcophytol A 46, and sarcophytol A
47 from Sarcophyton sp. in the western side of Mahengtang Island, Indonesia, with newly discovered anti-cancer activities against breast cancer MSF-7 ( $\left.\mathrm{IC}_{50}<30 \mathrm{mg} / \mathrm{L}\right)$ (Table 1, entries 42-47) [29]. Six new cembranoids 


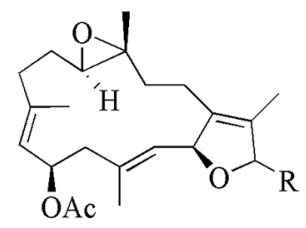

$\mathrm{R}=\mathrm{H}$ (11), OH (12)

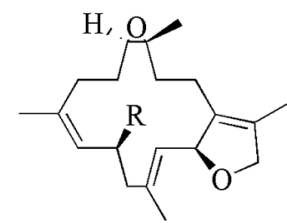

R= OAc (13), OH (14)

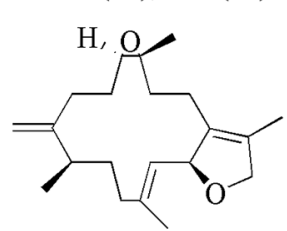

15

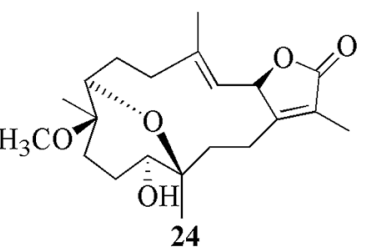

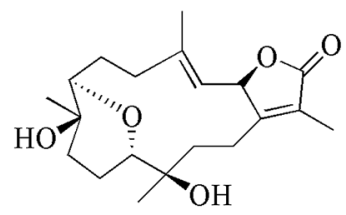

25<smiles></smiles>

26<smiles>CCC(C)=CCCC1=CC(CC(C)=CCCC(C)C(C)C)OC1=O</smiles>

97
$\mathrm{R}=\mathrm{CH}_{3}(\mathbf{1 0 0}), \mathrm{COOCH}_{3}(101)$
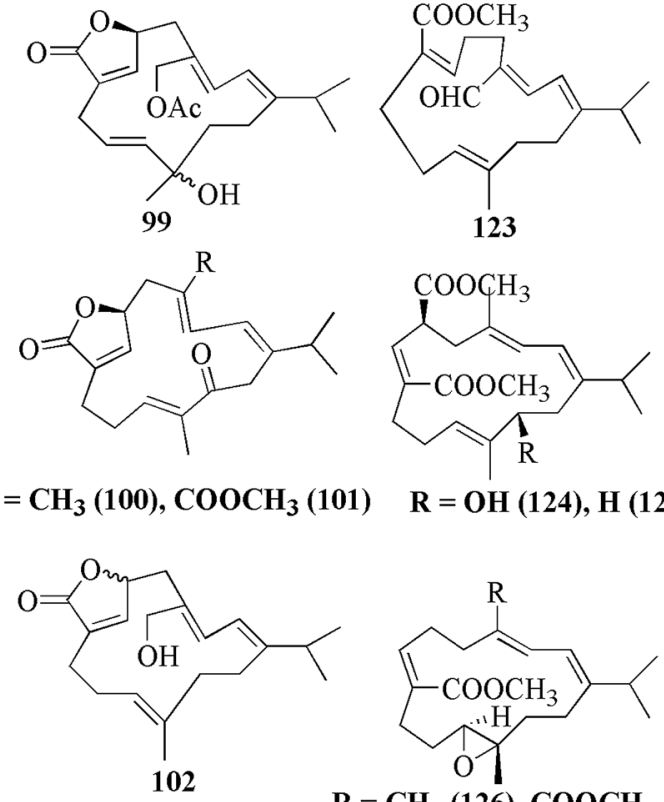

$\mathrm{R}=\mathrm{CH}_{3}(126), \mathrm{COOCH}_{3}(127)$

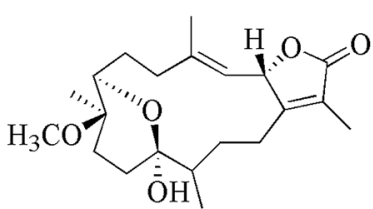

103

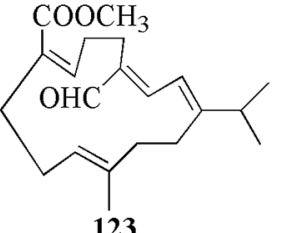

(COOCH

$\mathrm{R}=\mathrm{OH}(124), \mathrm{H}(125)$

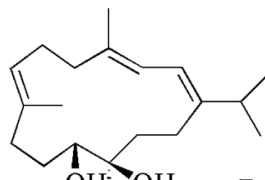

$\mathrm{OH} \equiv \mathrm{OH}$

128

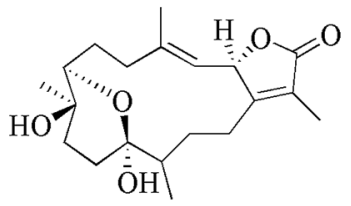

104

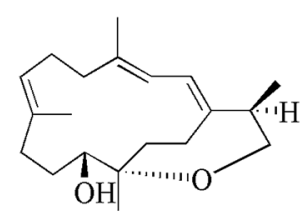

129

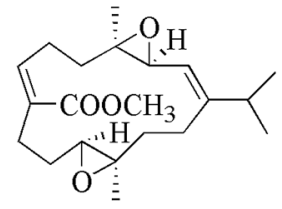

130<smiles>C=C(C)/C(=C/[C@H]1O[C@H]1CC/C=C\CC[C@@]1(C)O[C@H]1C)[C@H]1O[C@H]1C</smiles>

131

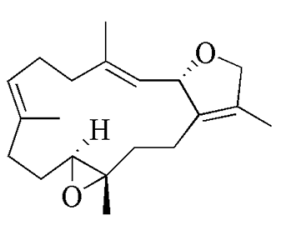

132<smiles>CCC(C)=CCCC(C)(O)CCCC(C)(O)C=CC(C)(O)C(C)C</smiles><smiles>[R]/C(=C/C=C(/C)CC/C=C(\C)CCC=C(C)C)C([R])C([R])C</smiles>

$\mathrm{R}=\mathrm{OH}(134), \mathrm{H}(135,136)$

$\mathrm{R}^{\prime}=-\mathrm{CH}\left(\mathrm{CH}_{3}\right)_{2}(134,136), \mathrm{CH}_{3}(135)$

$R^{\prime \prime}=$ H $(134,136)$, OH (135)

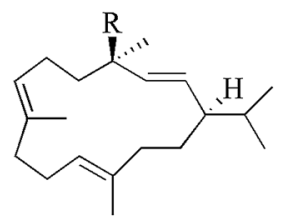

$\mathbf{R}=\mathbf{H}$ (137), OH (138)

Fig. 4 Cembranoids isolated from Sarchophyton ehrenbergi

related to 33-41, Sarcophytrols G-L 48-53 was also isolated from $S$. trocheliophorum from Yalong Bay, Hainan Province, China (Fig. 3). These compounds were tested for their inhibitory activity against PTP1B but showed no effect (Table 1, entries 48-53) [30]. Eight novel cembranetype diterpenoids were also discovered from Sarcophyton mililatensis isolated from Guangxi Autonomous Region, China, namely (+)-(6R)-6-hydroxyisosarcophytoxide 54, 
(+)-(6R)-6-acetoxyisosarcophytoxide 55, (+)-17-hydroxyisosarcophytoxide 56, sarcomililatins A-D 57-60, and sarcomililatol 61. Most of these compounds did not exhibit anti-cancer and anti-inflammatory activities, except for 57, which showed a moderate anti-inflammatory activity (Table 1, entries 54-61). Along with these newly discovered compounds, a known compound (+)-isosarcophytoxide 62 was also isolated and reported to have strong anti-cancer and moderate anti-inflammatory activity (Table 1, entry 62) [31].

Sarcophyton stellatum from the coast of Dongsha Atoll, Taiwan, was reported to yield seven new cembrane-based diterpenoids, stellatumolides A-C 63-65, stellatumonins A-B 66-67, and stellatumonone 68 (Fig. 5). Unfortunately, none of these compounds was found to have anti-cancer activity as tested (Table 1, entries 63-68) [33]. Within the same country, more precisely in Jihui Fish Port, a study reported five new cembranoids, cherbonolides A-E 69-73, a biscembranoid peroxide, bischerbolide peroxide $\mathbf{7 4}$, and a known cembranoid, isosarcophine $\mathbf{7 5}$, from Sarcophyton cherbonnieri [34].

Several studies isolated new compounds as well as known compounds with newly discovered biological activities from S. trocheliophorum in three different locations. From near Mahmieat of the Red Sea, Hurghada, Eas Egypt, two new pyrane-based cembrane diterpenoids 9-hydroxy-7,8-dehydro-sarcotrocheliol 76, and 8,9-expoy-sarcotrocheliol acetate 77, were tested for their antibacterial activity but were proved as inactive (Table 1, entries 76,77) [35]. From Yalong Bay, Hainan Province, China, six new highly oxidative cembranoids were discovered, sarcophytonolides S-U 78-80, and sartrolides 81-83. These new compounds were tested for their anti-diabetic activity along with a known compound sarcophytolide 84, but only 81 and 84 possessed the activity (Table 1, entries 78-84) [36]. A known biscembranoid, glaucumolide A 86, together with five new biscembranoids, bistrochelides A-E 87-91, were isolated from this species in Xisha Islands in the South China Sea. Following testing for their immunological activities, 86-89 were found to affect T-lymphocyte proliferation and differentiation, while 90-91 lacked this activity (Table 1, entries 86-91) [38].

A new cembranolide diterpene with anti-fungal activity, sarcophytonolide V 85, was discovered from Sarcophyton sp. in Sepanggar Bay, North Borneo [37]. In the Egyptian Red Sea off the coast of Hurghada, a study on Sarcophyton $\mathrm{sp}$. also isolated five new cembrane-type diterpenoids with moderate anti-cancer activity, namely 7-acetyl-8-episinumaximol G 92, 8-epi-sinumaximol-G 93, 12-acetyl-7, 12-epi-sinumaximol G 94, 12-hydroxysarcoph-10-ene 95, and 8-hydroxy-epi-sarcophinone 96 (Table 1, entries 92-96) [32]. A study on S. ehrenbergi from South China Sea reported five new cembranoids, sarcoehrenolides A-E 97-101, and a known cembranoid, ehrenbergol D 102. Compound 99 has not been tested for its biological activities, while the others were tested for their anti-cancer properties but were found to be inactive (Table 1, entries 97-101). Most of these compounds have anti-inflammatory activity, except for 101 [39]. Another study on the same species from the Egyptian Red Sea off the coast of Hurghada isolated three known cembrene diterpenoids, sarcoehrenbergilids D-F 103-105, which were reported to have anti-cancer activities (Table 1, entries, 103-105) [40].

Sarcophyton glaucum from Xisha Islands of the South China Sea was reported to yield nine new cembrane diterpenes, sarcoglaucins A-I 106-114, along with three known analogues, trochelioid 115, $7 \alpha$-hydroxy- $\Delta^{8(19)}$ deepoxysarcophine 116, and (-)-sartrochine 117 (Fig. 6). None of them possessed anti-cancer and anti-bacterial activities (Table 1, entries 106-114) [41]. A new diterpenoid, sarcomililate A 118, two new cembranoids, sarcomililatols A-B 119-120, and two known related diterpenoids, yalogene $A$ 121 and sarcophytol M 122, were isolated from Sarcophyton mililatensis in Xigu Island, Hainan Province, China. Most of them were active as an anti-cancer agent, except for $\mathbf{1 2 0}$ (Table 1, entries 119-121) [42].

Another study on S. ehrenbergi from Weizhou Island, Guangxi Province, China, isolated eleven new cembrane diterpenes, sarcoehrenins A-I 123-131, (2S,11S,12S)-isosarcophytoxide 132, and sarcoehrenin J 133. These compounds were tested for their anti-inflammatory potentials; however, only 129 and 130 were active. In addition, this study also discovered new anti-inflammatory activity on five known compounds within the same species, 13S)-cembra1,3,7,11-tetraen-13-ol 134, (+)-sarcophtol 135, cembrene-C 136, (1R,4R,2E,7E,11E)-cembra-2,7,11-trien-4-ol 137, and (1S,4R,2E,7E, 11E)-cembratrien-4-ol 138 (Table 1, entries 123-138) [43]. Lastly, a known trans-diol derivative of sarcophine, (7S, 8R)-dihydroxy-deepoxysarcophine $\mathbf{1 3 9}$ was isolated from S. glaucum in Dabah, Ras Sudr, and Sharm El-Sheikh, Red Sea Coast, and revealed that 139 exhibited anti-cancer and neurological activities (Table 1, entry 139) [44].

Furthermore, S. digitatum which cultured in the National Museum of Marine Biology and Aquarium, Taiwan contained seven biscembranoids and one cembranoid. Four out of seven biscembranoids were unreported compounds namely sardigitolides A-D 140-143 (Fig. 7). The other three biscembranoids were reported before and namely sarcophytolide L $\mathbf{1 4 4}$ and glaucumolides A-B 145-146. The only known cembranoid collected from this species namely isosarcophytonolide D 147. The reported cembrane-type diterpenoid from S. digitatum was reported to display various anti-cancer and anti-inflammatory activities [45]. Another study reported nine cembranoids from Sarcophyton tenuispiculatum which culture at Kaohsiung Medical University, Taiwan. The three novel cembranoids sarcotenusenes A-C 148-150 were mostly 
<smiles>C=C(C)C(=CC=C(C)CCC1(C)OC1(C)CCC=C(C)C)CCC</smiles>

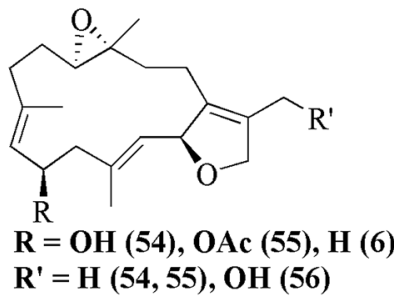<smiles>[B]C(C=CC(C)(O)CC[C@H](O)C(C)(O)CCC1=C(C)OC(=O)C1C)=CC1OC1=O</smiles>

O,<smiles>C=C1CC[C@@H]2O[C@]2(C)CCC2=C(C)C(=O)O[C@@H]2/C=C(\[As])CC[C@@H]1OO</smiles>

58<smiles></smiles>

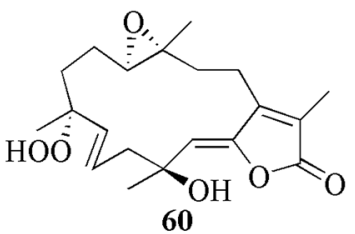

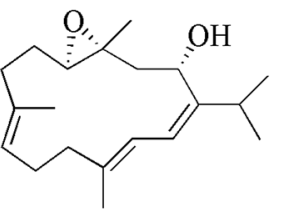

61

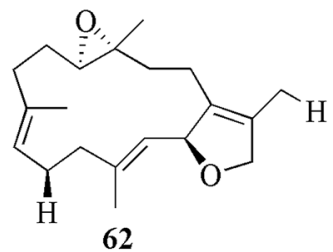<smiles>[R]C1C=C(C)CC[C@]1(O)O[C@@H]1CCC2=C(C)C(=O)O[C@@H]2C=C(C)C1</smiles>

$$
\mathrm{R}=\mathrm{OH}(69), \mathrm{OOH}(\mathbf{7 0})
$$

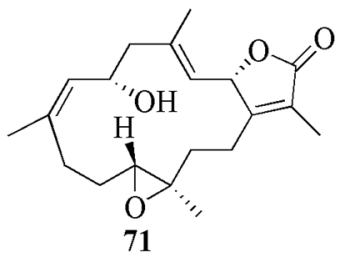

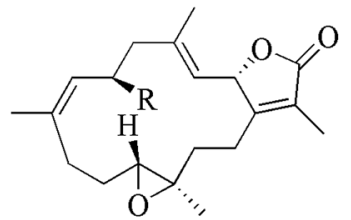

$\mathbf{R}=\mathbf{O H}(72), \mathbf{O O H}(73)$

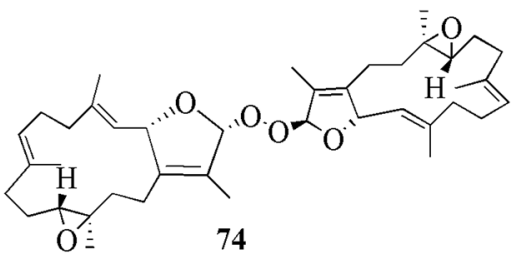<smiles></smiles>

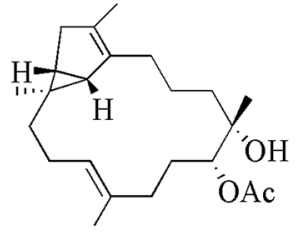

118<smiles>CC1=C(CC[C@@H]2O[C@H]2C)/C(=C/[C@@](C)(O)CC/C=C2/CC[C@H]2C)OC1=O</smiles>

168<smiles>[R]C(C)(O)CC/C(=C\C=C(/C)CC/C=C(/C)C(C)(C)O)C(C)(C)O</smiles>

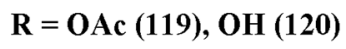<smiles>C=C(C)/C(=C/C=C(/C)CC/C=C(\C)CCC=C(C)C)CC</smiles>

121<smiles>CCC(C)=CCC/C(C)=C\CC/C(C)=C/CC(O)C(C)C</smiles>

122<smiles>[R20]C(C)(/C=C/CC(C)=CC1OC(=O)C(C)=C1CCC1(C)CO1)CC</smiles>

$\mathbf{R}=\mathbf{H}(\mathbf{1 6 3}), \mathrm{OH}(164)$

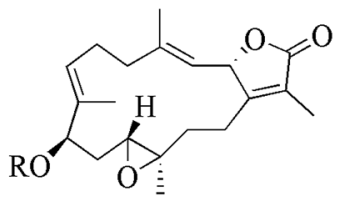

$\mathbf{R}=\mathbf{H}(\mathbf{1 6 5}), \mathrm{OH}(\mathbf{1 6 6 )}$
167

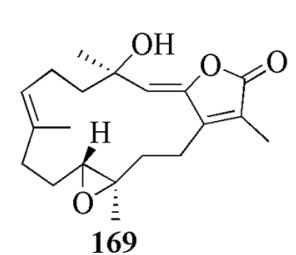

Fig. 5 Cembranoids isolated from Sarcophyton stellatum (32), Sarcophyton mililatensis (54-62, 118-122), Sarcophyton cherbonnieri (69-75, 163-169)

inactive in PPAR- $y$ transcription factor assay; cytotoxic assay towards MCF-7, MDA-MB-231, HepG2 and HeLa cell line; and inflammatory assay. Moreover, $(2 \mathrm{~S}, 7 \mathrm{~S}$, 8S)-sarcophytoxide 151, (2S, 7R, 8R)-sarcophytoxide
152, sarcophytonin F 153, 3,4-dihydro- $4 \alpha$-hydroxy- $\Delta^{2}$ sarcophine 154, A hydroperoxide obtained by autoxidation of dihydrofuranocembranoid 155, and (+)-7 $\alpha, 8 \beta$ dihydroxydeepoxysarcophine $\mathbf{1 5 6}$ were also displayed 


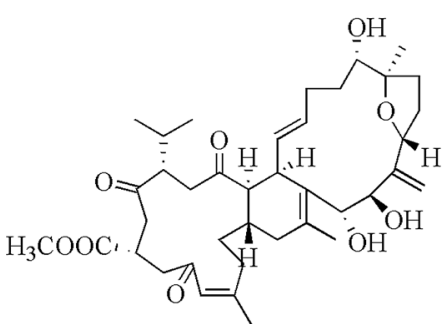<smiles>CC(C)[C@]1(O)/C=C/C(C)(O)CC[C@@H](O)C2(C)CCC=C(CCC2=O)OC1</smiles>

23

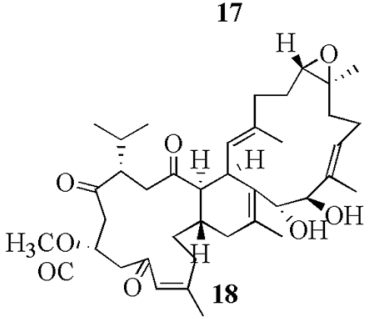<smiles>CCC(/C=C/CC[C@@]1(C)OC(/C(C)=C/C=C(/C)C(C)C)CC1=O)C(=O)OC</smiles>

19<smiles>CC(C)C1(O)/C=C/C(C)(O)CCC(=O)C2(C)CCC=C(CCC2=O)C(=O)O1</smiles>

20<smiles>CC(C)C12CCC3=CCCC(C)(O)C(=O)C3=CCC1(C)O2</smiles>

21

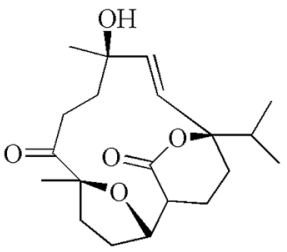

22
28<smiles>CC1=CCC[C@](C)(O)[C@@H](O)CC/C(C)=C/[C@H]2OC(=O)C(C)=C2CC/C(C)=C\1</smiles>

29<smiles>CC1=C[C@H]2OC(=O)C(C)=C2CC[C@](C)(O)CCC[C@@](C)(O)[C@@H](O)CC1</smiles>

30

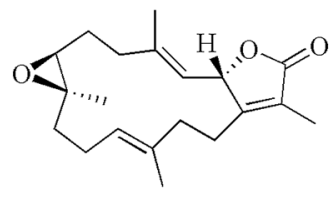

31

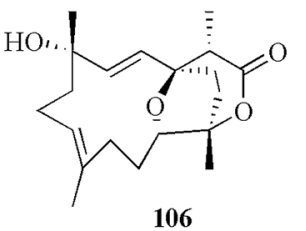

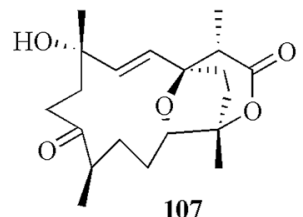

107

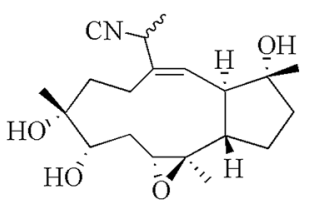<smiles>CC1=CC(O)C/C(C)=C/[C@H]2OC(=O)C(C)=C2CC[C@@H]2O[C@@H]12</smiles>

113<smiles>CC1=C[C@H](O)/C=C(/C)CC[C@@H]2O[C@H]2CCC2=C(C)C(=O)O[C@@H]12</smiles>

$\mathrm{HO}$,<smiles>C=C(CC)CCCC(C)=CCC1=C(C)C(=O)O[C@@H]1C=C(C)CCCC</smiles>

109

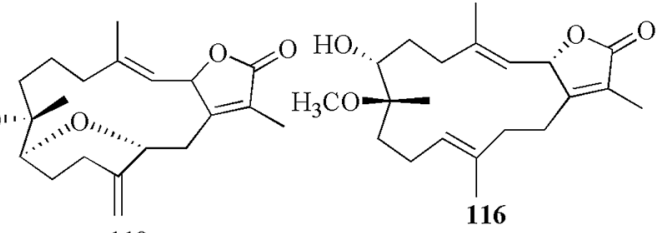

110

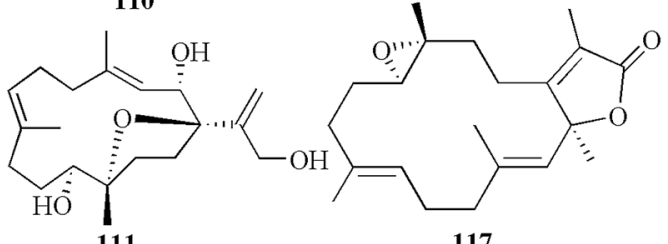

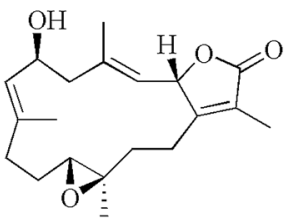

112

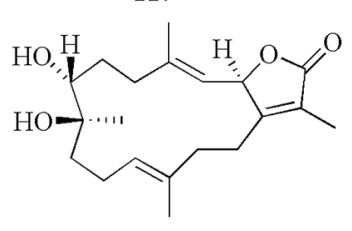

139

Fig. 6 Cembranoids isolated from Sarchophyton elegans (17-23, and Sarchophyton glaucum (27-31, 106-117, 139)

various results on the abovementioned assay [46]. Additionaly, six cembranoids were isolated from S. roseum collected in Dahab, Red Sea, Egypt. The new cembranoid sarcoroseolides A-D 157-160 and the known cembranoid 2-epi-sarcophine 161 and 2R,7R,8R-dihydroxydeepoxysarcophine $\mathbf{1 6 2}$ were being assessed for its anti-inflammatory and anti-cancer activities [47]. Lastly, Sarcophyton cherbonnieri collected from Jihui Fish Port, Taiwan, contained seven novel cembranoid that possessed various anti-inflammatory activities through inhibition of superoxide anion generation and elastase release, namely cherbonolides F-L 163-169 [48]. 


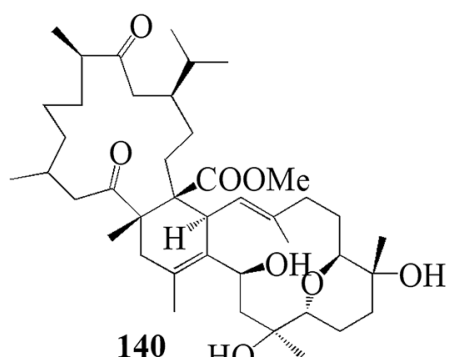

$140 \mathrm{HO}^{\prime \prime \prime " ~}$
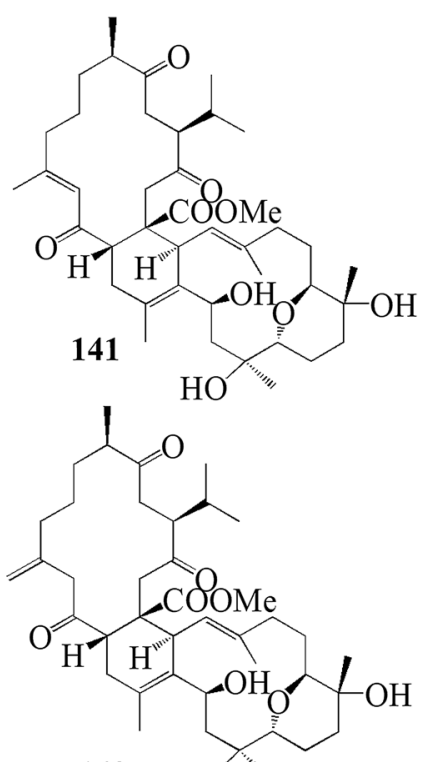

$142 \mathrm{HO}$
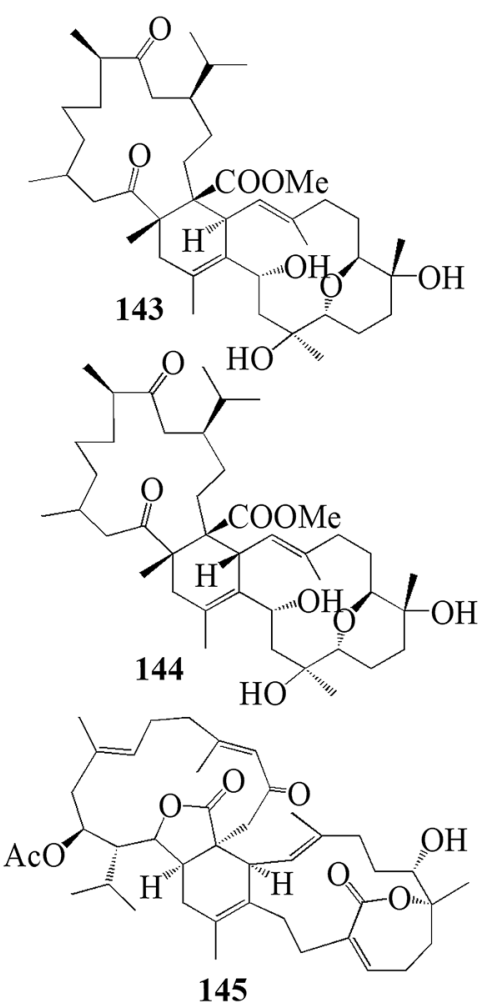
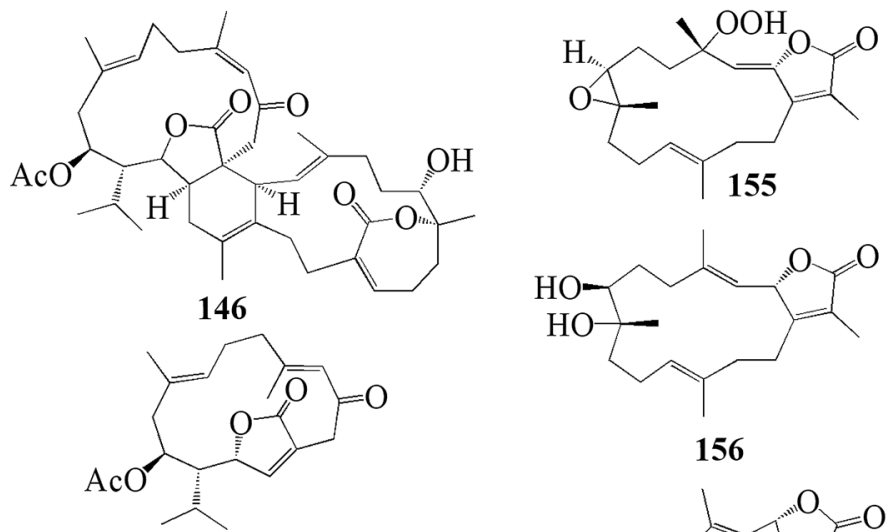

156

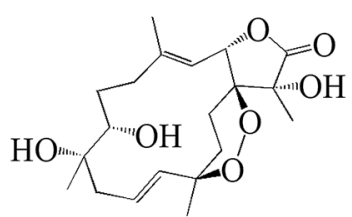

157

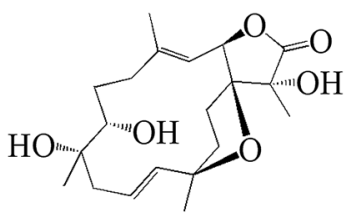

158
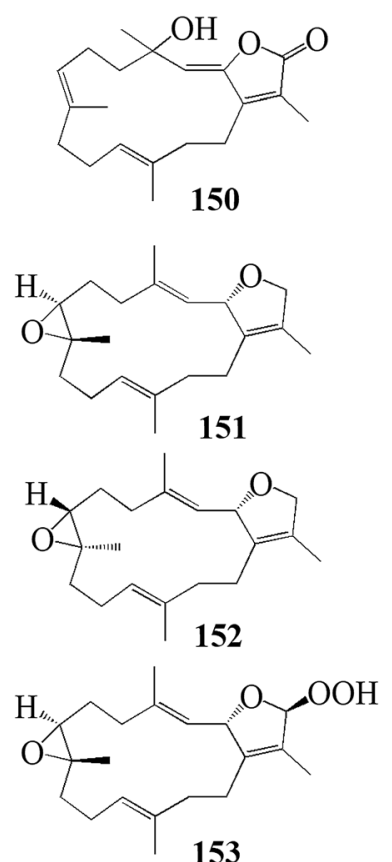

153

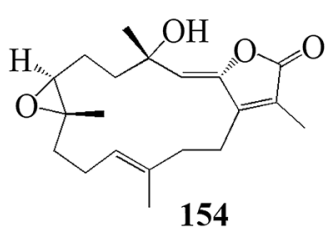

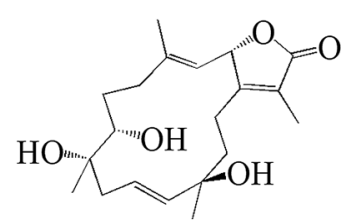<smiles>CC(=CC1OC(=O)C(C)=C1CCC(C)(O)[C@H](O)CCC(C)(O)C(=O)CCC(=O)[AlH2])C(=O)O</smiles><smiles>CCC(C)=CC1OC(=O)C(C)=C1CC/C(C)=C/CCC1OC1(C)C</smiles>

161

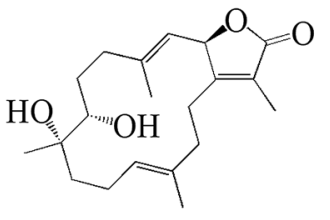

162

Fig. 7 Cembranoids isolated from Sarchophyton digitatum (140-147), Sarchophyton tenuispiculatum (148-156) and Sarchophyton roseum (157-162) 


\subsection{Cembranoids from Genus Sinularia}

The present study reported 42 cembranoid compounds isolated from Sinularia sp. collected from various geographical areas (Fig. 8). Twenty-nine of those were new compounds and the other 13 were previously known compounds with newly discovered activities. One of the new compounds was newly discovered and had not been thoroughly tested for their biological activities.

Soft coral Sinularia erecta from the South China Sea yielded three new norcembranoids, sinulerectols A-C 170-172 [49], whereas a new-non tested cembranoid diterpene named isodecaryiol $\mathbf{1 7 3}$ was collected from Madagascar Sinularia gravis [50]. Three new non-active cembranoids from Taiwan were isolated from S. nanolobata namely nanolobols A-C 174-176 along with one known biologically active cembranoid sinulariol C 177 [51]. Sinularia compacta from the South China Sea contained three new cembranoid diterpenes namely 5-epi-sinuleptolide $\mathbf{1 7 8}$, michaolide $\mathrm{F}$ 179, and 20-acetylsinularolide B 180 [53]. S. sandensis was reported to produce a known compound 7-acetylsinumaximol B 181 [55]. Kamada et al. isolated Sinularia sp. from Sabah, Malaysia and discovered a new cembranoid named sinularolide F 182 and a known cembranoid named denticulatolide 183 [56]. Taiwanese S. flexibilis produced seven compounds, three of which were new compounds with no biological activities named flexibilisins D-E 184-185 and flexibilisolide H 186 (Table 2, entries 17-19). The other four compounds were known compounds with various biological activities, namely 11-dehydrosinulariolide 187, 11-episinulariolide acetate 188, (S)-14-deoxycrassin 189, and sinulariolide 190 [57].

A known cembrane, sandensolide 191 was isolated from aquacultured S. flexibilis in Pingtung, Taiwan [59]. Qin et al. isolated two new and two known compounds from Chinese Sinularia sp., named sinulins C-D 192-193 and 5-epi-sinuleptolide 178, (1R,3S,4S,7E,11E)-3,4-epoxycembra-7,11,15-triene 194, with 192 being reported as not showing any biological activity as tested (Table 2, entries 29-31) [54]. Eight cembranoids were isolated from Chinese S. flexibilis, three of which were newly discovered. The three new compounds were categorized as polyoxygenated cembranoids (or flexibilide-like cembranoids) and named xidaosinularides A-C 165-167. The known compounds were categorized as polyoxygenated cembranoids and included 11-dehydrosinulariolide 187, 11-epi-sinulariolide acetate 188, sinulariolide 190, sinuladiterpene I 198, and flexilarin B 199 [58] Tables 3 and 4.

Sinularia sp. from Xisha Islands yielded four new cembranoids named $1 E, 3 E, 7 E,-11$-hydroxy-12-methoxy-1-isopropyl-4,8,12-trimethyl-icyclotetradeca-1,3,7triene 200, 3E,7E-11-hydroxy-12-methoxy-1-isopropenyl-4,8,12-trimethyl-icyclotetradeca-3,7-diene 201,
$1 E, 3 Z, 7 E,-11$-hydroxy-12-methoxy-1-isopropyl-4,8,12trimethyl-icyclotetradeca-1,3,7-triene 202, and 1Z,3Z,7E,11-hydroxy-12-methoxy-1-isopropyl-4,8,12-trimethyl-icyclotetradeca-1,3,7-triene 203. The study showed that 201 and $\mathbf{2 0 3}$ had no biological activity [60]. Sinularia scabra from Hainan, China, contained ten cembranoids. Six of them were novel compounds, namely, xiguscabrates A-B 204-205, xiguscabral A 206, xiguscabrols A-B 207-208, and 8-epixiguscabrol B 209, with 204 and 206 not yet found to have biologically activity as tested. The known compound were sinulariol C 177, sinulariolide 190, $(2 R, 11 S, 12 S)$-isosarcophytoxide 210, and (-)-14-deoxycrassin 211 [52]. Figure 8 shows the structure of cembranoids isolated from Sinularia sp.

Sinularia crassa from West Island, South China Sea contained four new and one known cembrane-type diterpenoids; sinulacrassins A-C 212-214, ent-xishaflavalin G 215, and S-(+)-cembrane A 216 (Fig. 9). Compound 212 was not tested for its activity, while compound 213 and 216 showed a potential inhibitory effect towards $\alpha$-Glucosidase [61]. Lastly, six novel compounds were reported from Sinularia humilis collected from Ximao Islands, Hainan, China namely humilisins A-F 217-222. Compound 222 was the only reported diterpenoid that possessed biological activity by decreasing NO level in anti-inflammatory assay [62].

\subsection{Cembranoids Reported from Genus Lobophytum}

The present study reported 47 cembranoid compounds isolated from Lobophytum sp. collected from various geographical areas (Figs. 10, 11). Twenty-nine of those were new compounds and the other 18 were previously known compounds with newly discovered activities. Twelve of the new compounds were newly discovered and have not been thoroughly tested for their biological activities.

Lobophytum crassum was found to produce different cembranoid compounds. Cembrene A 223, a new cembranoid diterpene, was isolated from Red Sea Lobophytum sp. in Jeddah [63]. Ten new cembranoids and three known cembranoids were isolated from Hainan Lobophytum crassum in Meishan, China. Locrassumin A 224 and G 230 were the new compounds showing biological activities, whereas locrassumins B-F 225-229, (-)-laevigatol B 231, (-)-isosarcophine 232, and (-)-7R,8S-dihydroxydeepoxy sarcophytoxide $\mathbf{2 3 3}$ were the new compounds that have not been tested yet for their biological activity. Meanwhile, three known compounds with new activities were ent-sarcophine 234, sarcophytonolide O 235, and ketoemblide 236 [64]. Three new-non tested compounds, lobophylins F-H 237-239, were isolated from Dongsha Atoll L. crassum [65].

Another study discovered a Japanese Lobophytum sp. that produced one new casbane-type diterpenoid and two 


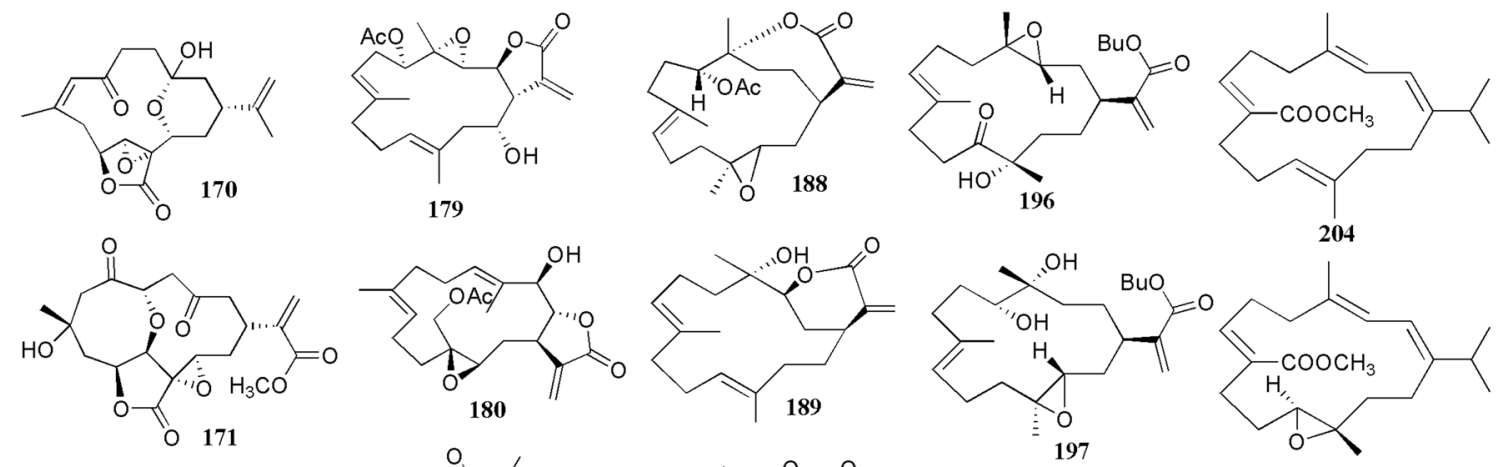

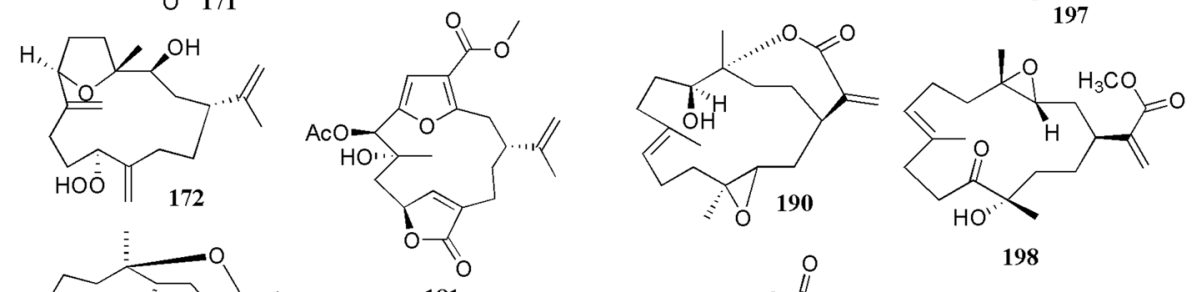

$\underbrace{C_{172}}_{\text {HOO }}$
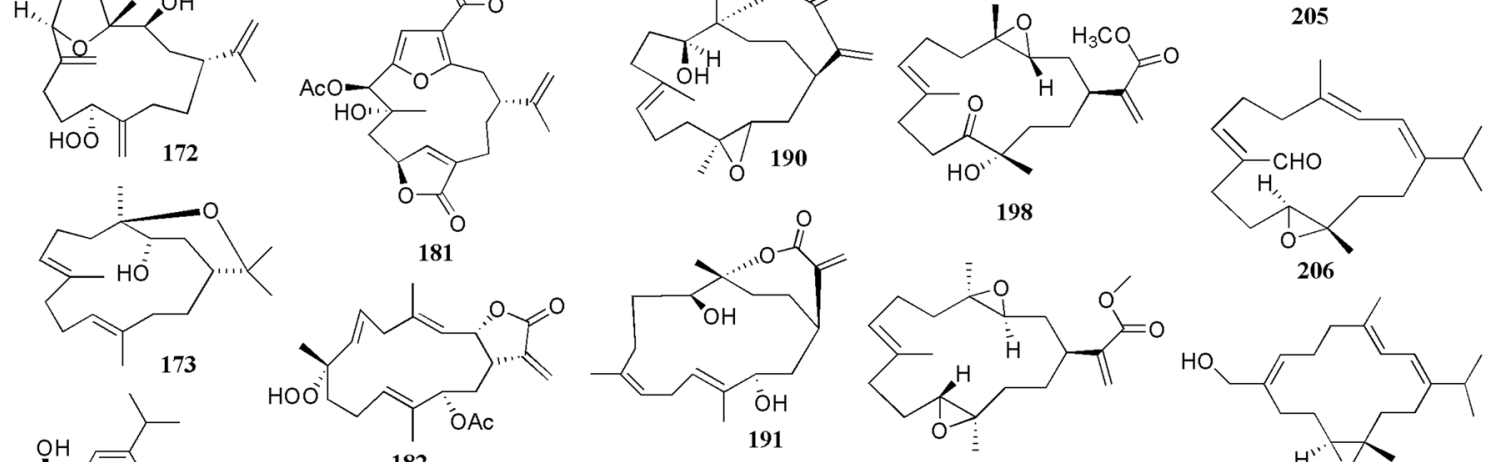

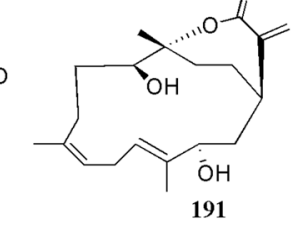

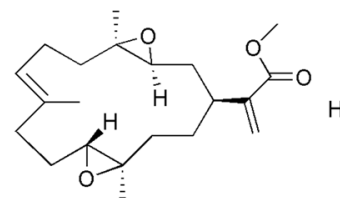

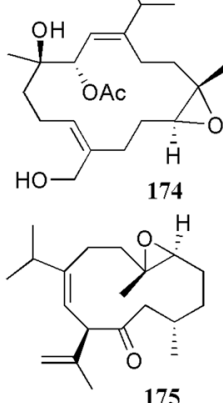

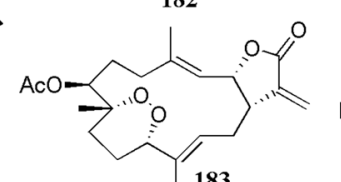

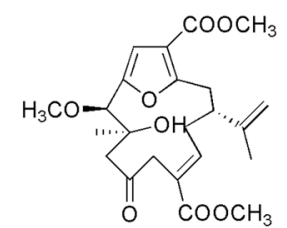

192<smiles></smiles>

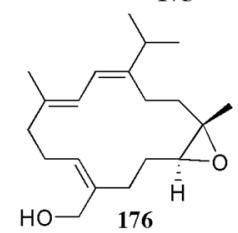

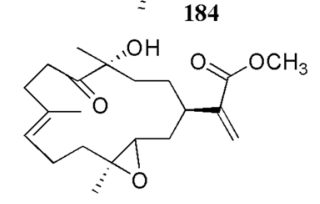

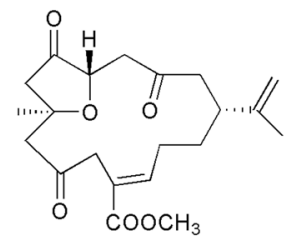

193
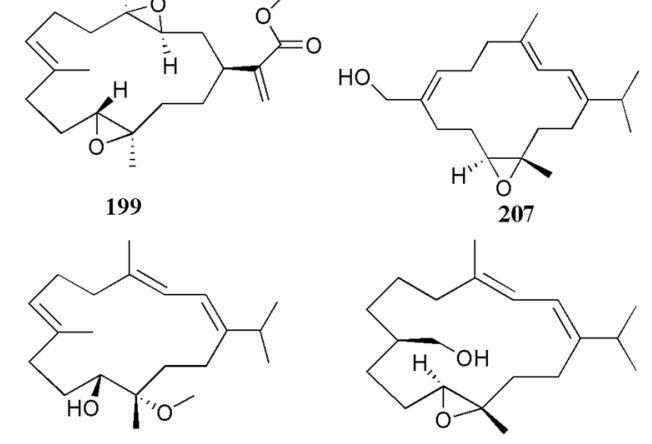

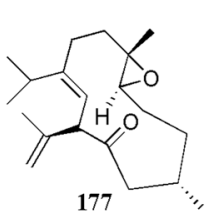

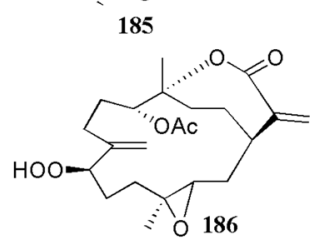

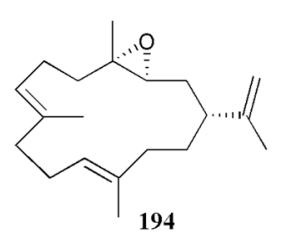<smiles>C=C(C)C(C/C=C(/C)CC/C=C\CC[C@H](C)O)COC</smiles><smiles>CC(=CC=C(CCC1(C)CCCO1)C(C)C)CCCC1CCCC1</smiles>

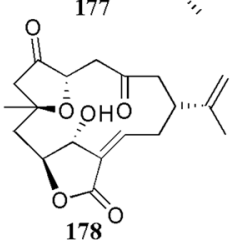

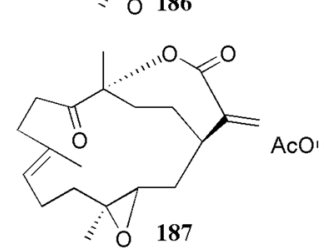<smiles>C=C(C(=O)O)C(CCC1OC1CCC1OC12CCCO2)CC1CCCC1O</smiles><smiles>CCC[C@@](O)(CC/C(=C\C1=CCC=C1)C(C)C)OC</smiles><smiles>CC(=CC=C(CCC(C)(C)O)C(C)C)CCCCC1CCCCC1O</smiles>

Fig. 8 Cembranoids reported from Sinularia erecta (170-172), Sinularia gravis (173), Sinularia nanolobata (174-177), Sinularia compacta (178-180), Sinularia sandensis (181), Sinularia sp. (182-183,

192-194, 400-203), Sinularia flexibilis (184-191, 195-199) and Sinularia scabra (204-211)
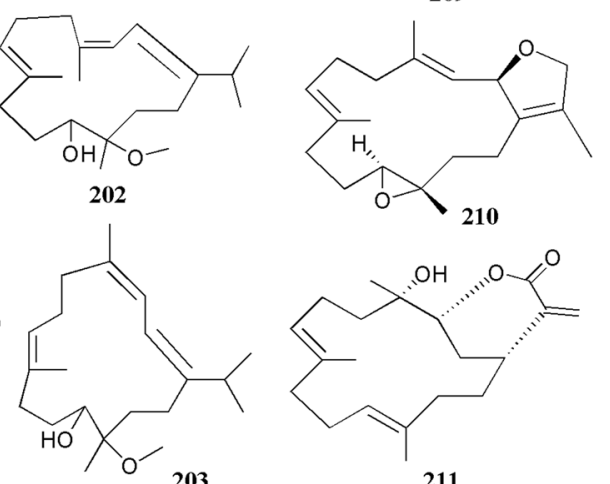

211 


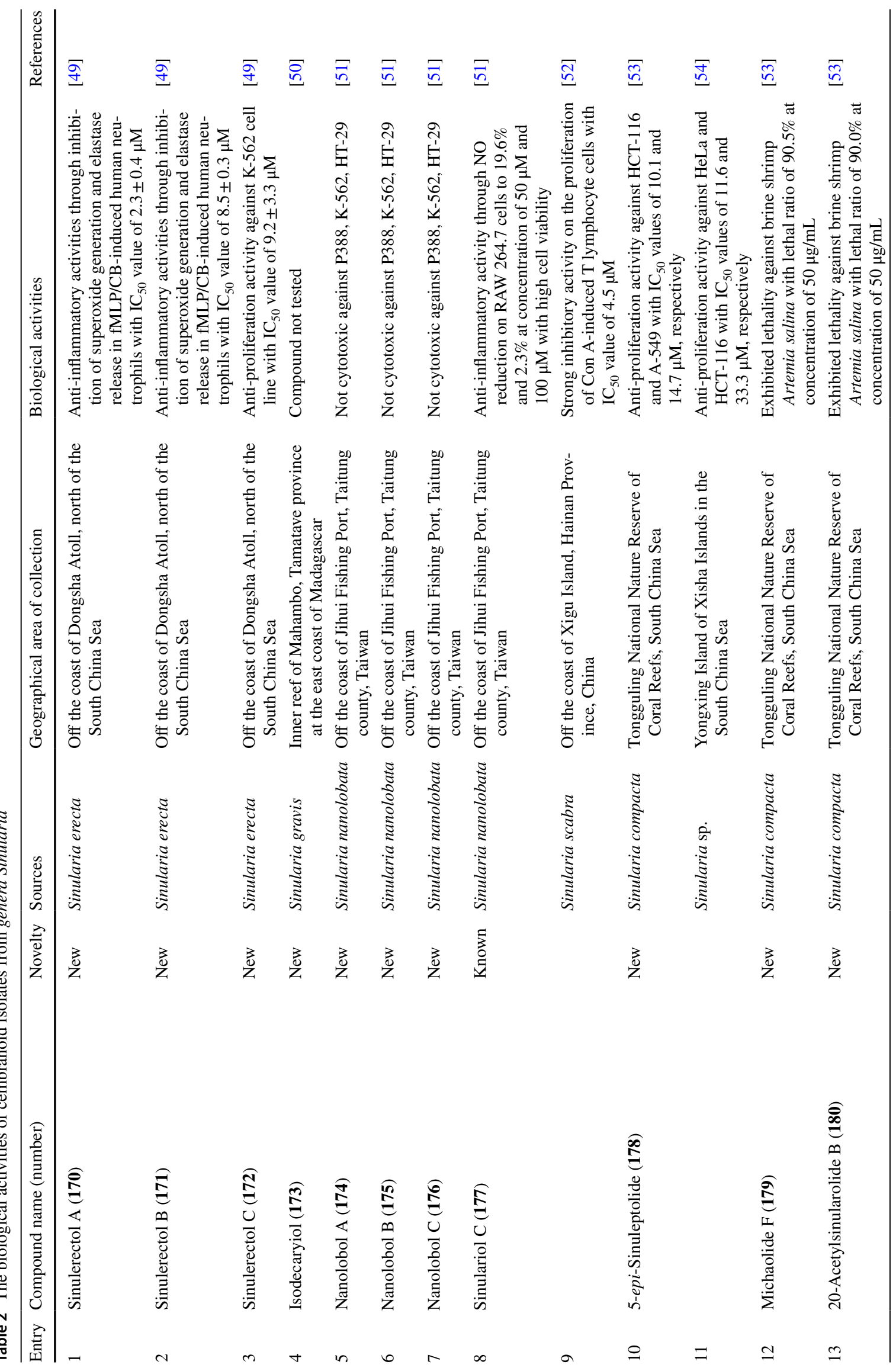




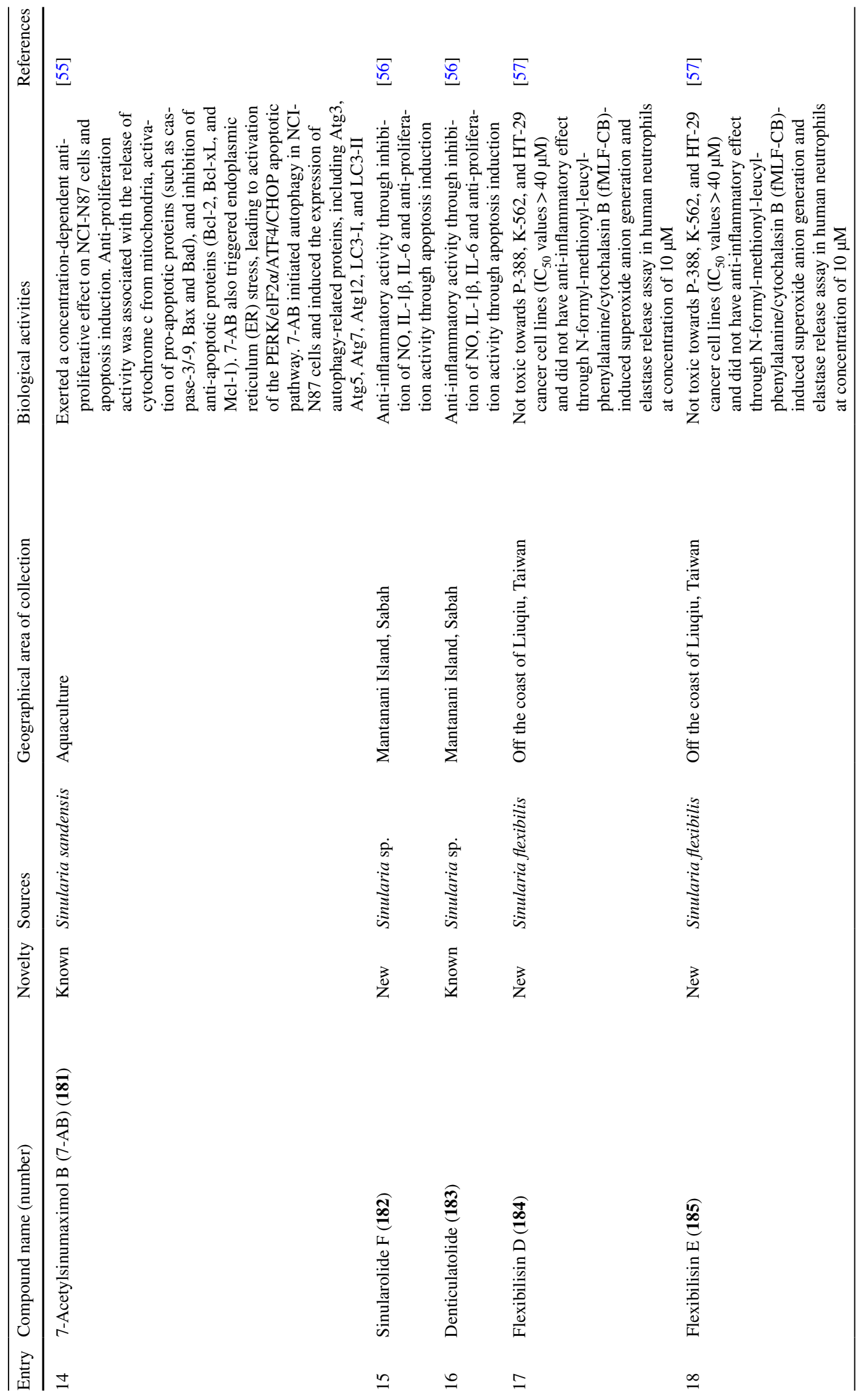




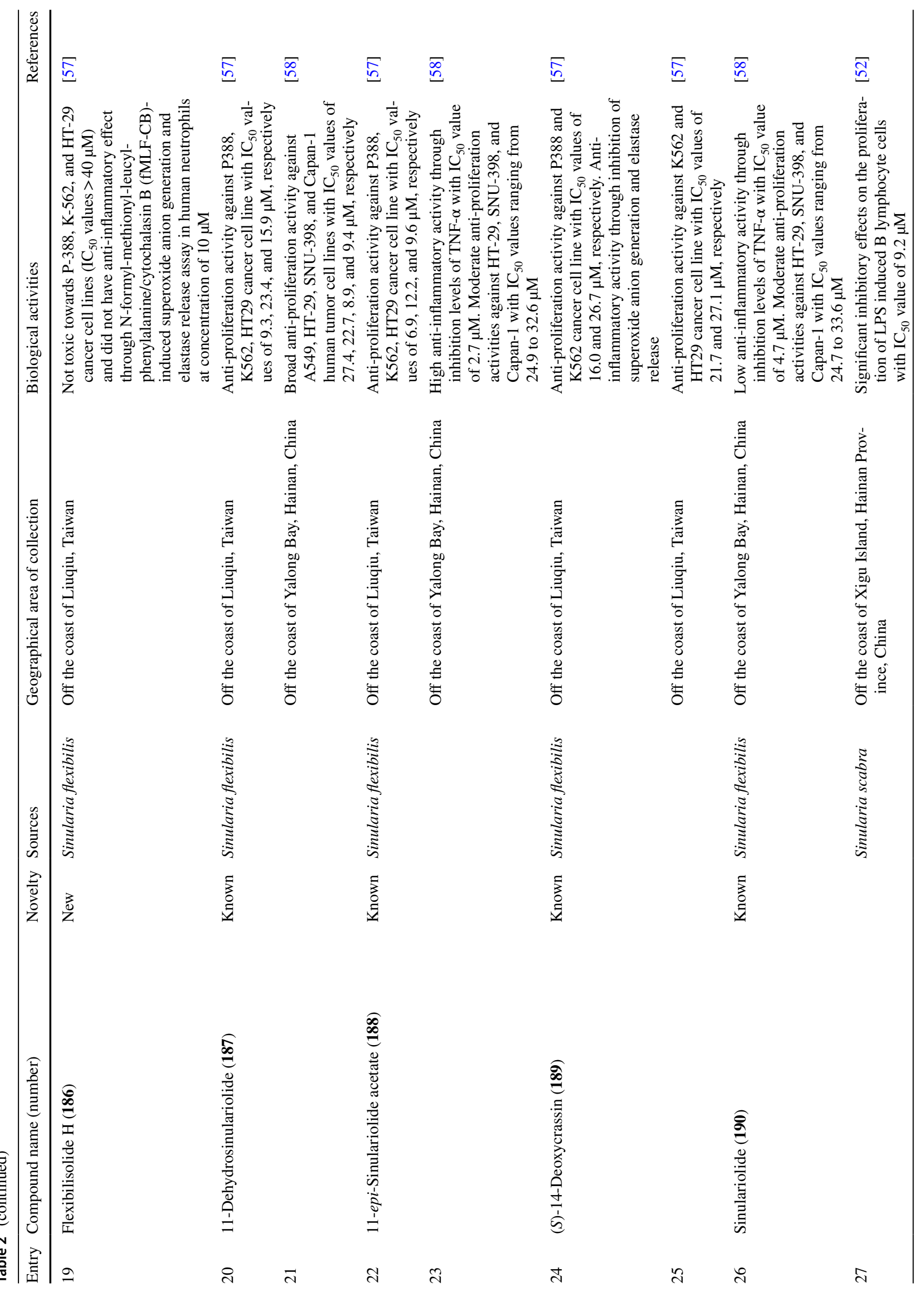




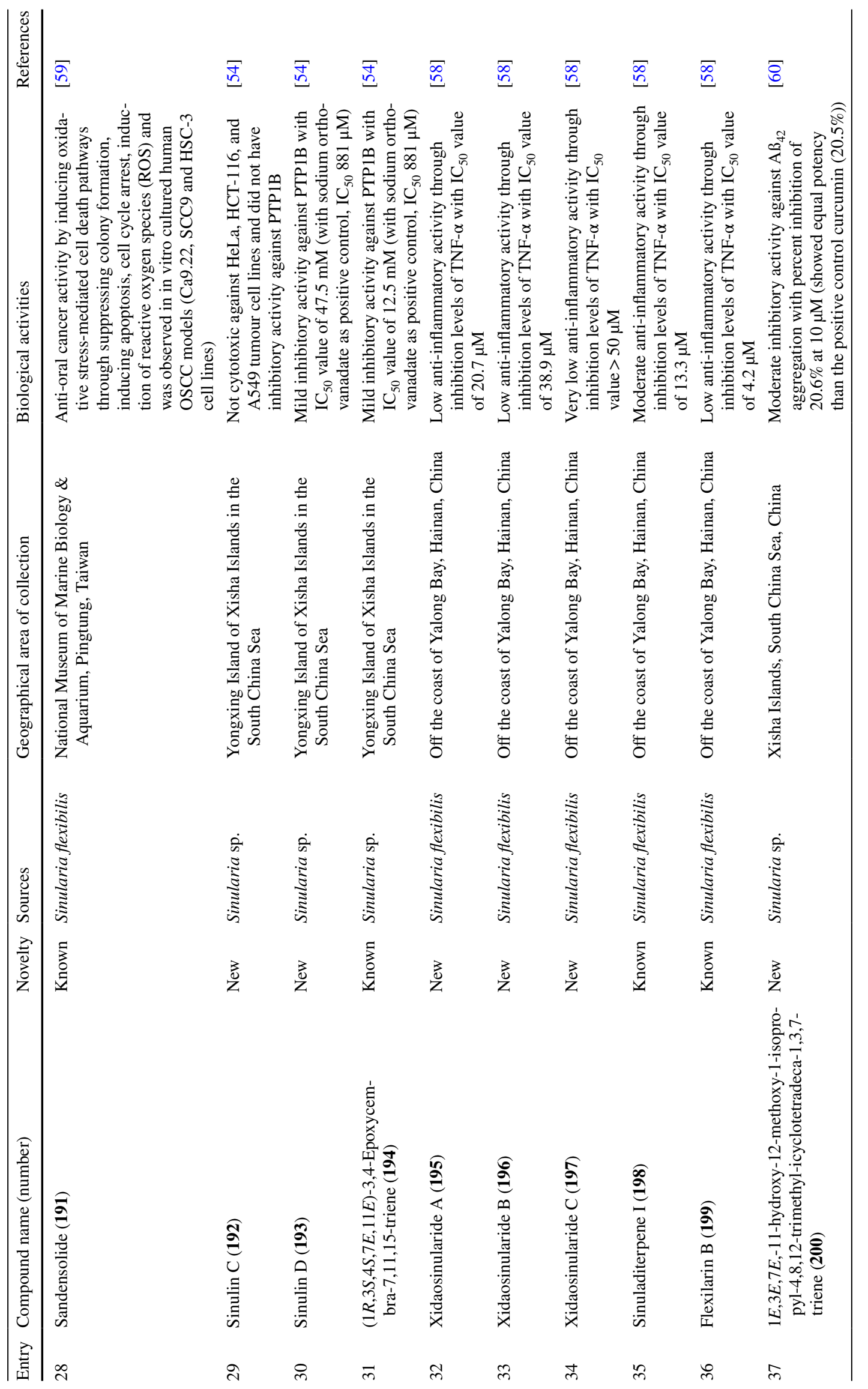




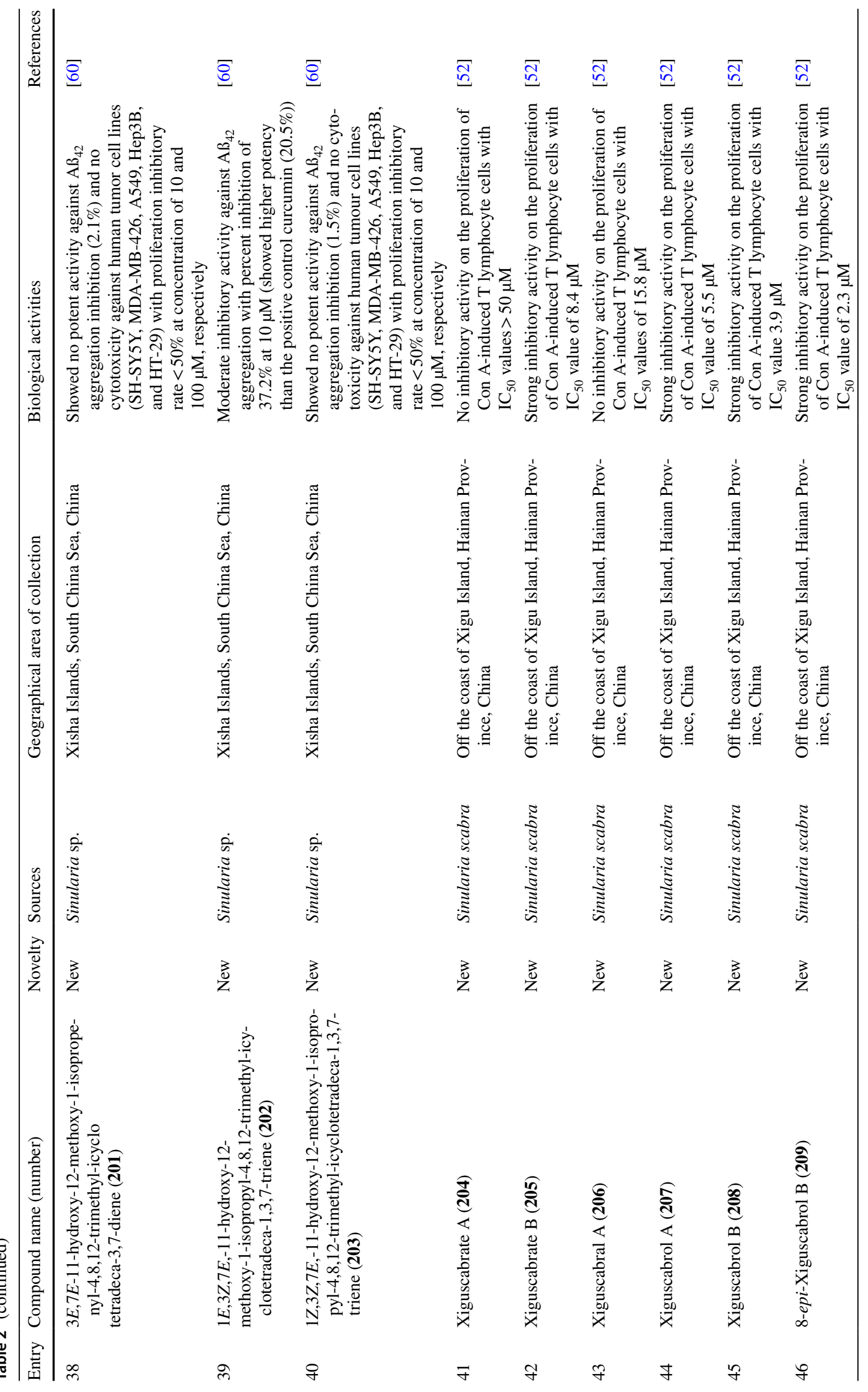




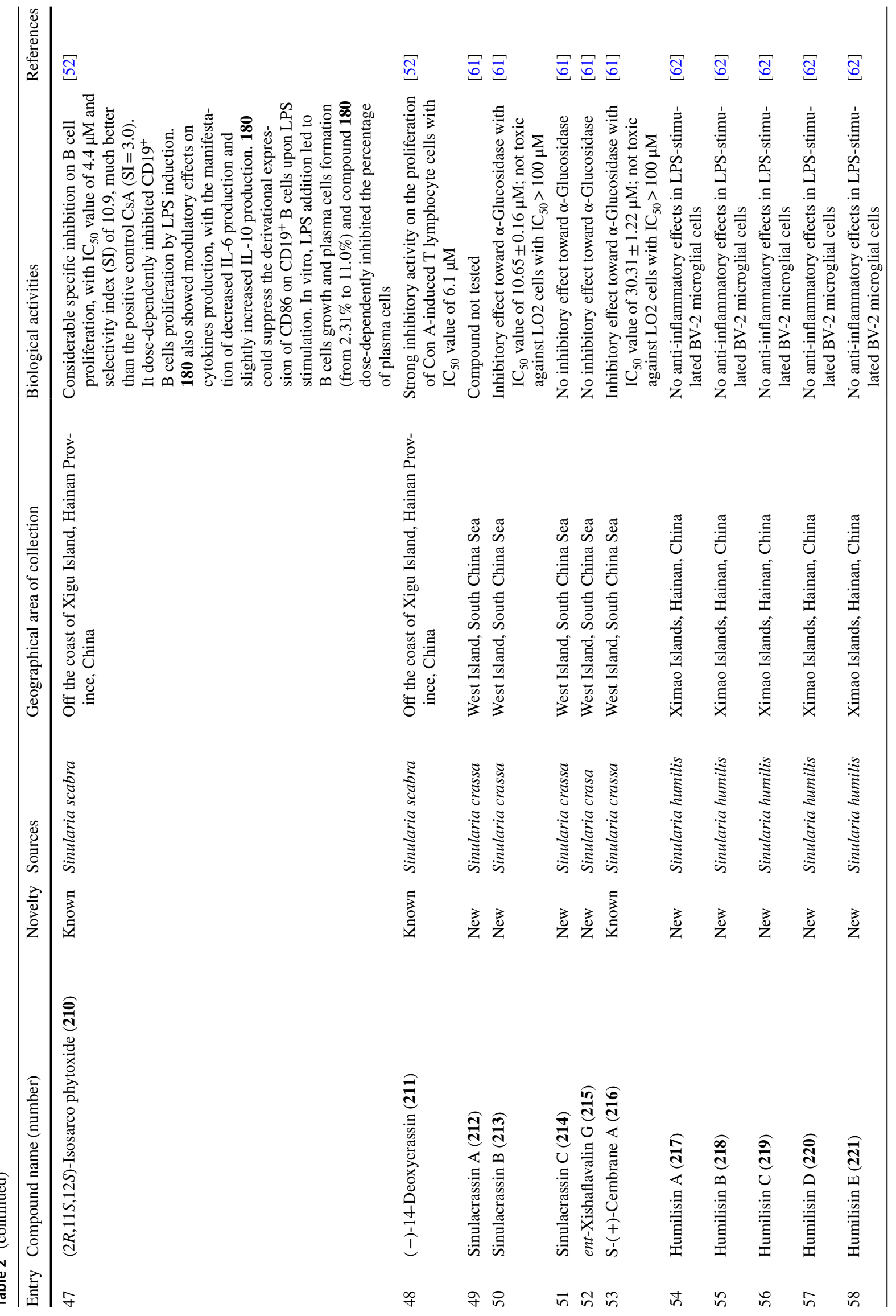


new cembrane diterpenoids (compound 1-3 240-242) with various biological activities. Moreover, it also produced two known compounds, grandilobatin B 243 and sinugibberol 244 [66]. The latter study reported that five cembranoids was obtained from from Lobophytum crassum collected from the coast of Pingtung, Taiwan. Two of them were new compounds named lobophyolides A-B 245-246, whereas three were known compounds called 16-methoxycarbonyl cembrene A 247, sinarone 248, and sinaluriol D 249 [11]. In the same sampling area, twelve compounds were reported from the aquaculture Lobophytum crassum. Two compounds were new (culobophylin D 250, and culobophylin E 251) while the others were known compounds including lobocrassin C 252, lobophylin 253, crassocolide E 254, sarcocrassocolide 255, 13-acetoxysarcocrassocolide 256, sarocrassocolide $M$ 257, $(R)$-14-deoxycrassin 258, lobocrassin B 259, sarcocrassocolides F-G 260-261 [67]. Recently, a known compound 13-acetoxysarcocrassocolide $\mathbf{2 5 6}$ was also reported from the same aquacultured Lobophytum crassum by Liu et al. [68].

Three new unnamed cembranolide diterpenes (compound 4-6 262-264) with various biological activities were isolated from Irabu Island Lobophytum sp. which have [69]. Furthermore, three new capnosane-type diterpenoids with no biological activities named lobophytrols A-C 265-267 were isolated from Lobophytum sp collected in Weizhou Island, China [70]. Lastly, new macrocyclic cembranoids lobophytolins A-B 268-269 isolated from Lobophytum sp. were collected from Xisha Islands, China, with both compounds not showing any biological activities [71]. Lastly, seven unreported cembranoid was isolated from Lobophytum sp. collected from the Xisha Island, Hainan, China. The new cembrane-type diterpenes, namely, lobophytolins C-I 270-276, displayed various anti-cancer activity towards HT-29, Capan-1, A549, and SNU-398 cancer cell line. Moreover, they also exhibited a weak inhibitory effect of $\mathrm{XBP}-$ Splicing on B16-F10 tumor cells [72].

\subsection{Cembranoids from Other Soft Corals Species}

The present study reported 80 cembranoid compounds isolated from other than the above-mentioned soft coral species collected from various geographical areas (Fig. 10). Fiftyfive were new compounds and the other 25 were previously known compounds with newly discovered activities. Ten of the new compounds were newly discovered and have not been thoroughly tested for their biological activities.

In 2016, a known cembranoid named claudieunicellin S 277 was isolated from Cladiella tuberculosa collected from Penghu Archipelago waters, Taiwan [73]. Six new briaranetype diterpenoids were isolated from Taiwanese Briareum sp. named briarenolides ZI-ZVI 278-283. Among these, 279 and 283 showed biological activities [74]. Later in 2016, three new cembranoids were isolated from Nephthea 


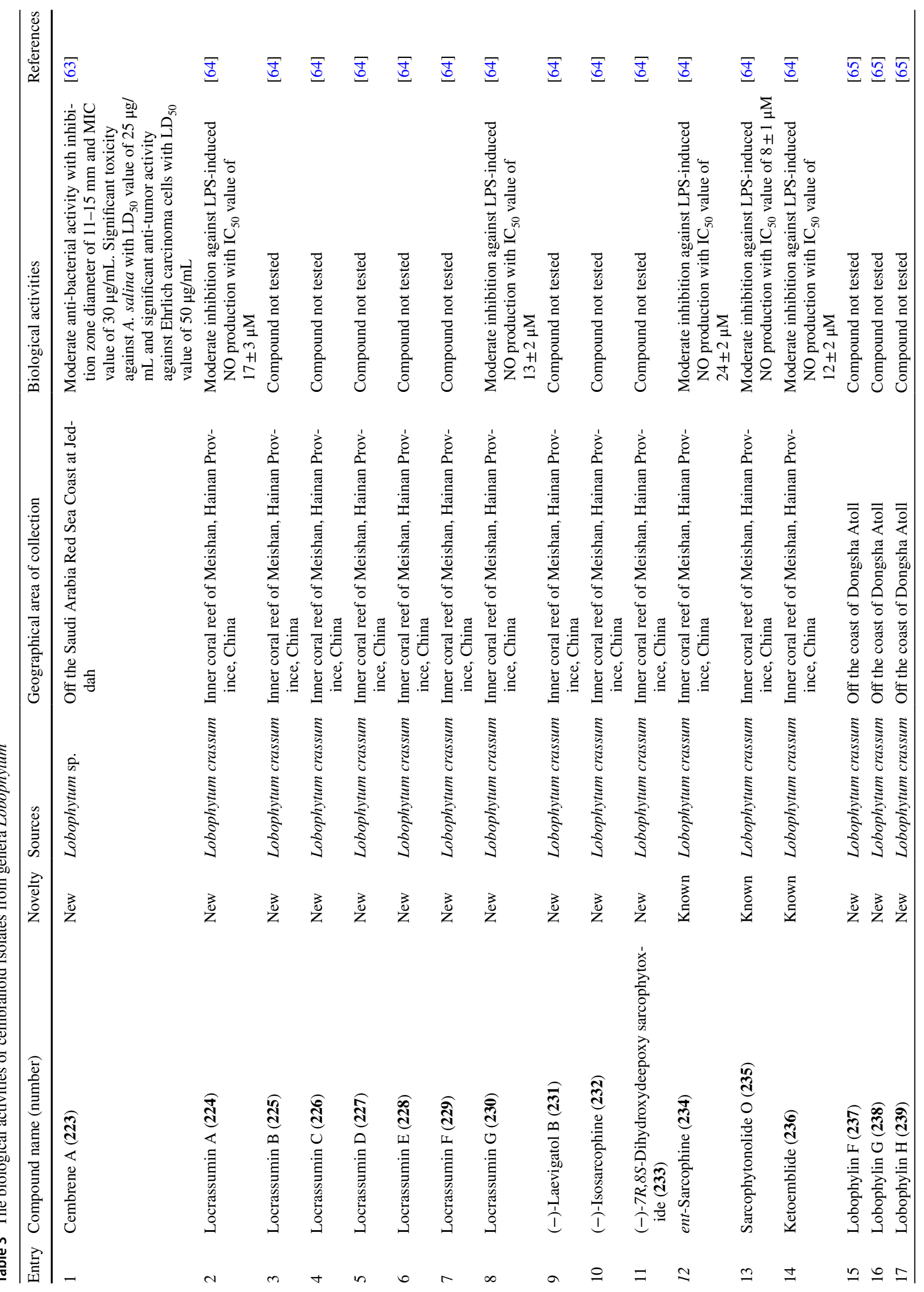




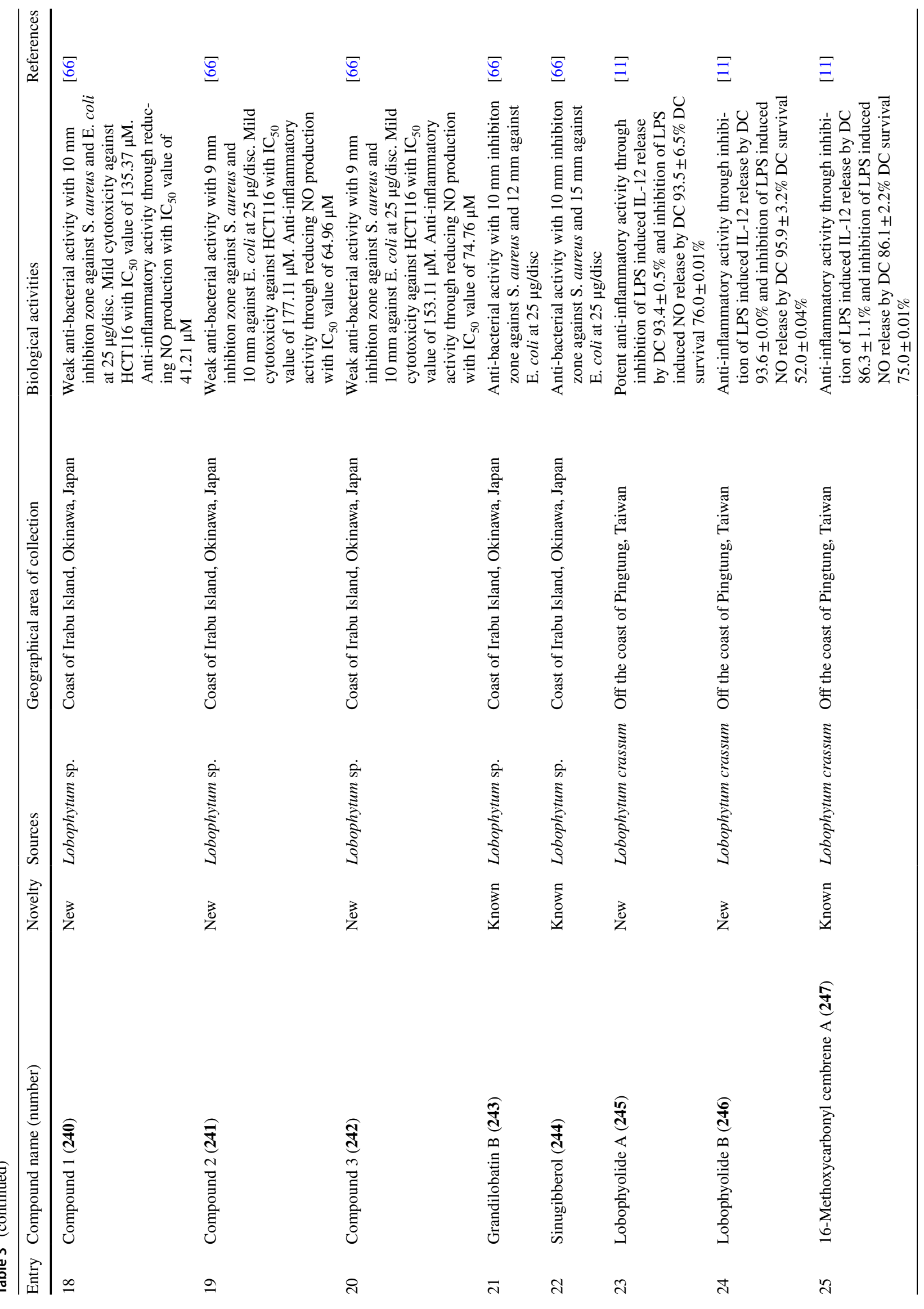




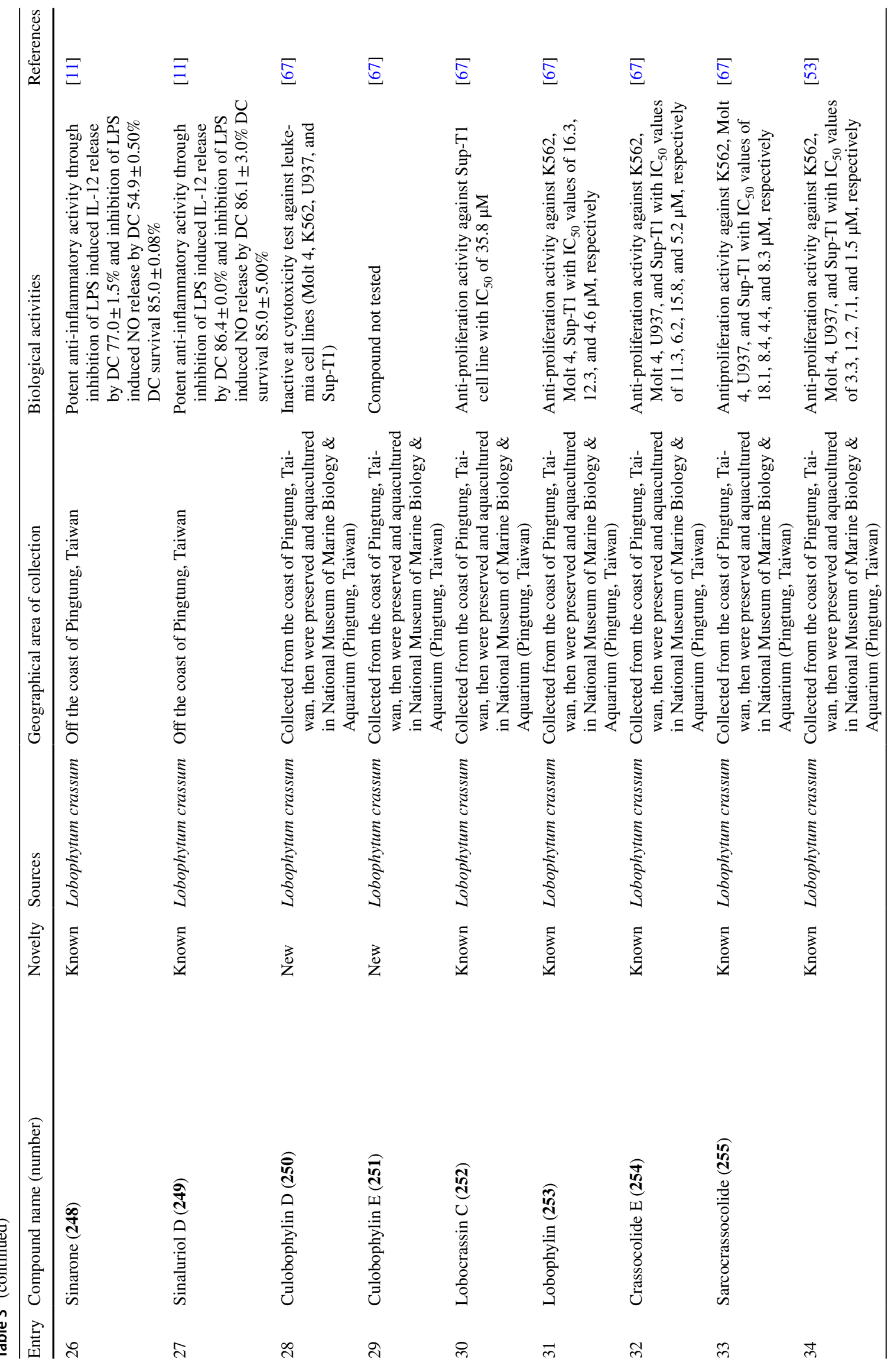




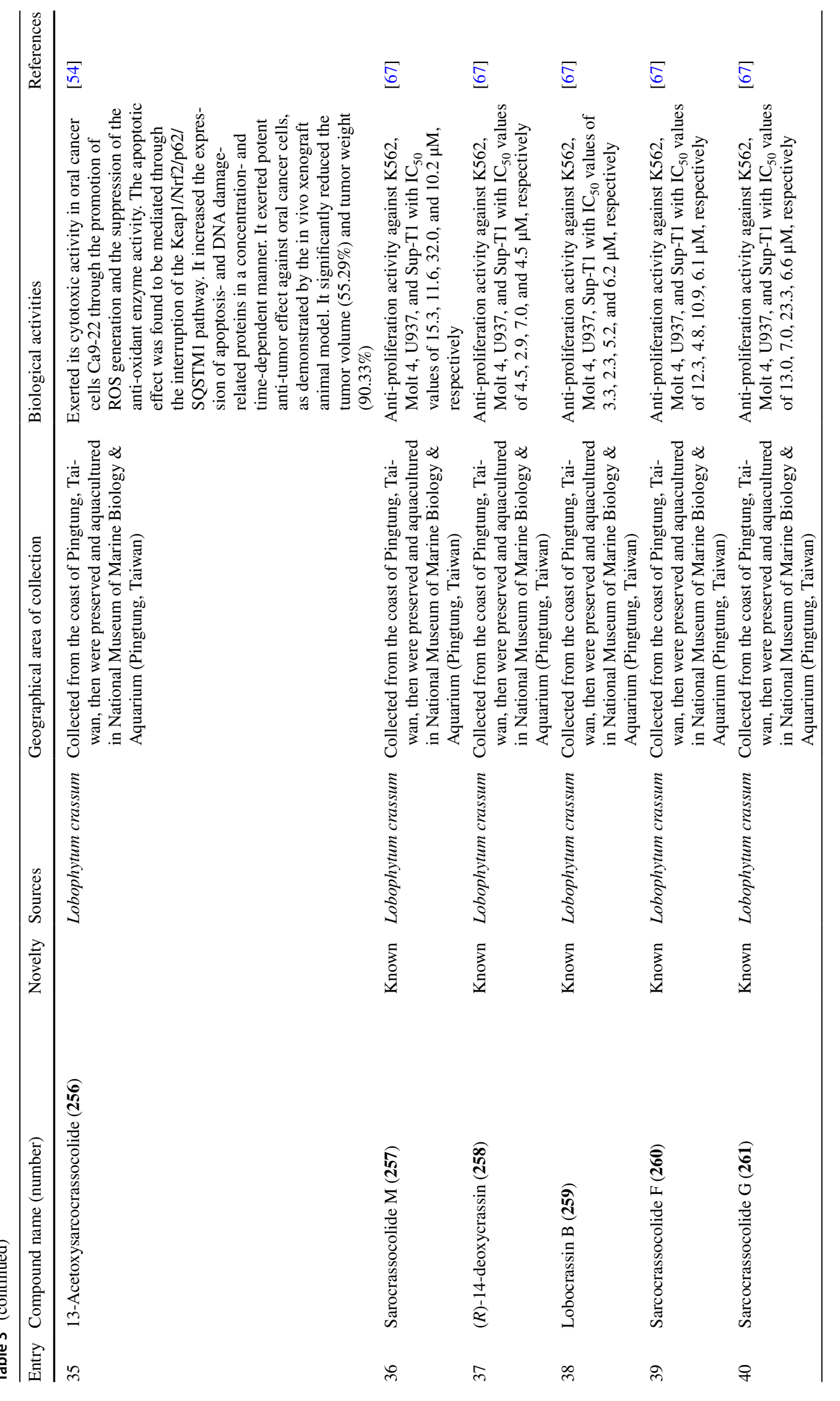




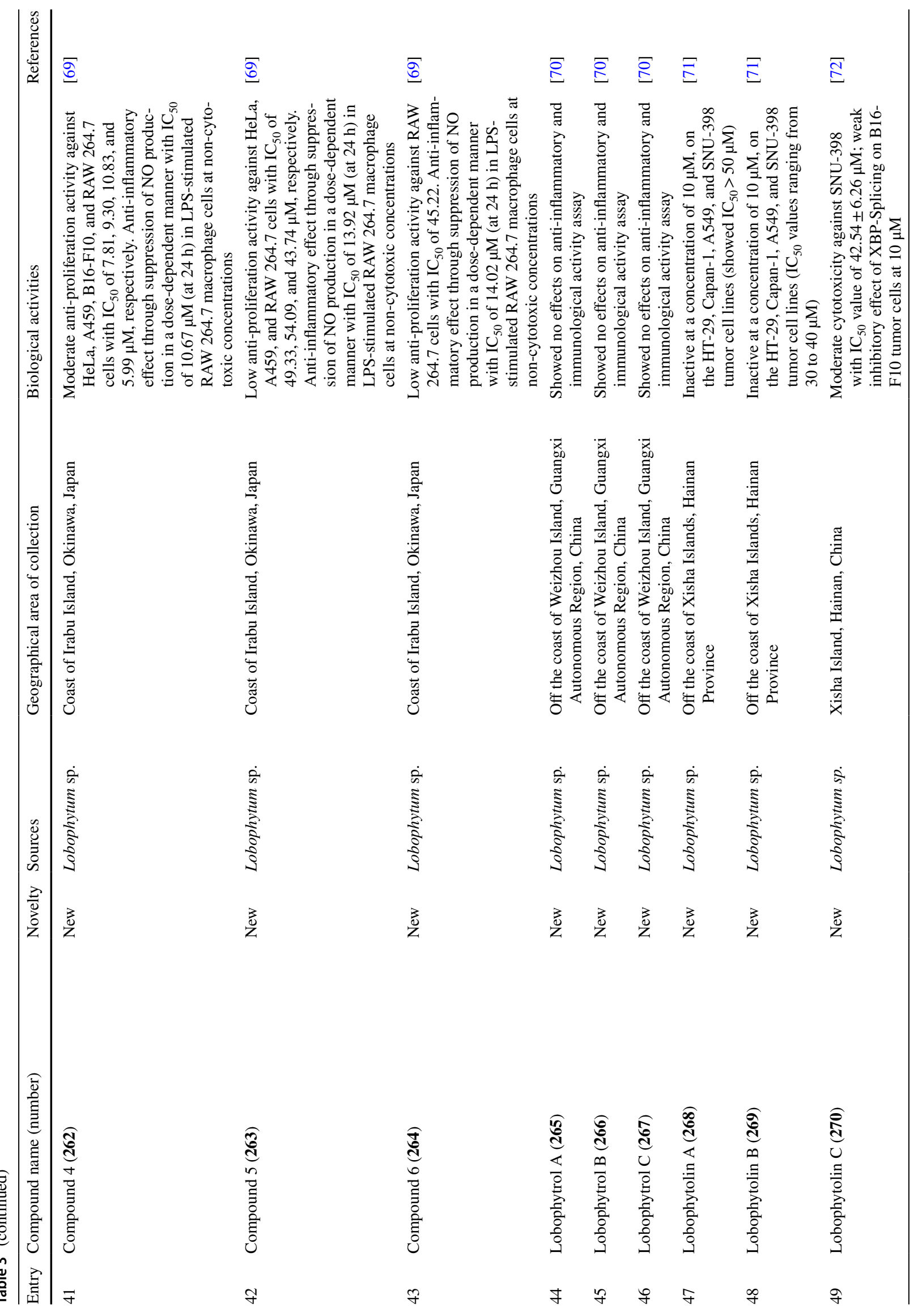


Cembranoids of Soft Corals: Recent Updates and Their Biological

285

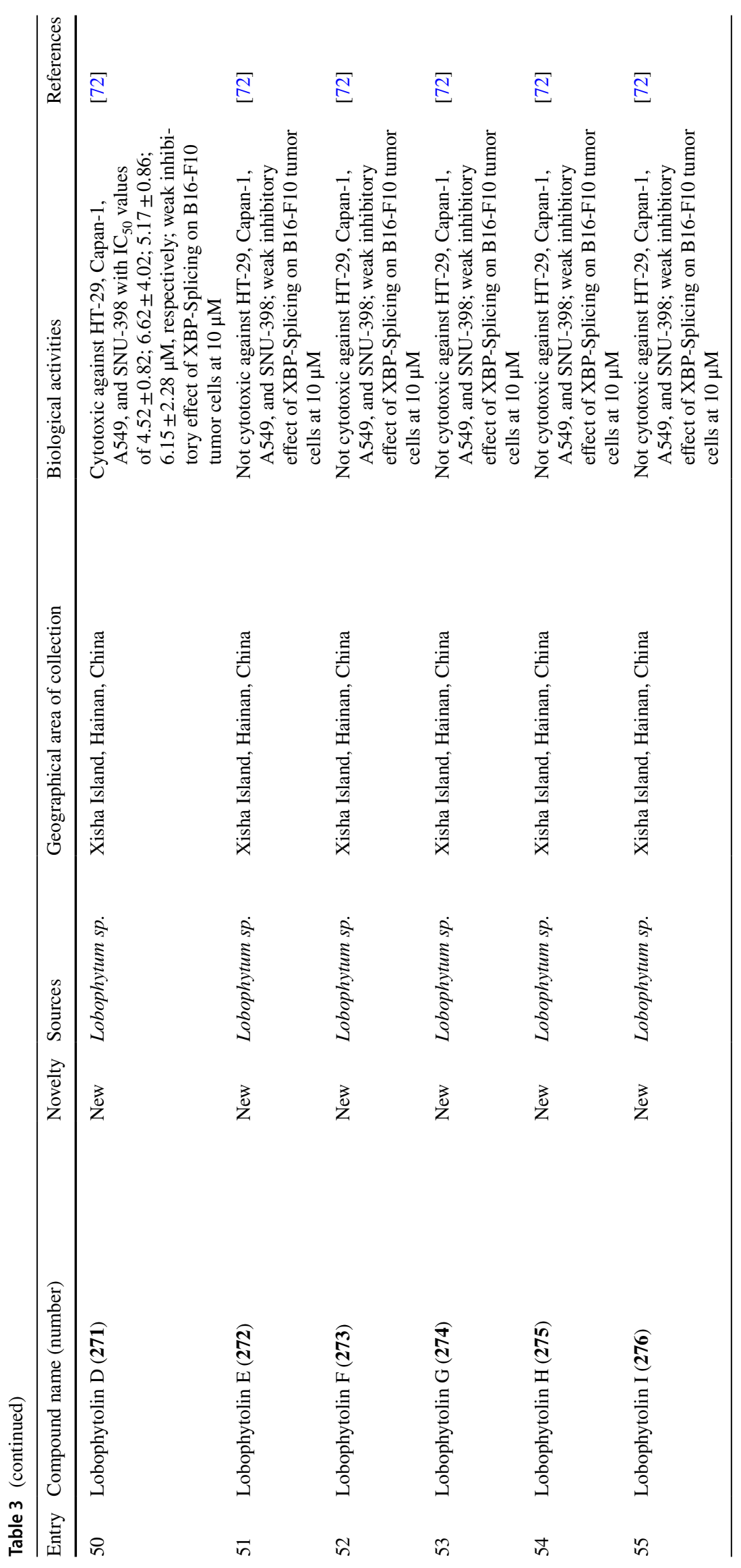

Springer 


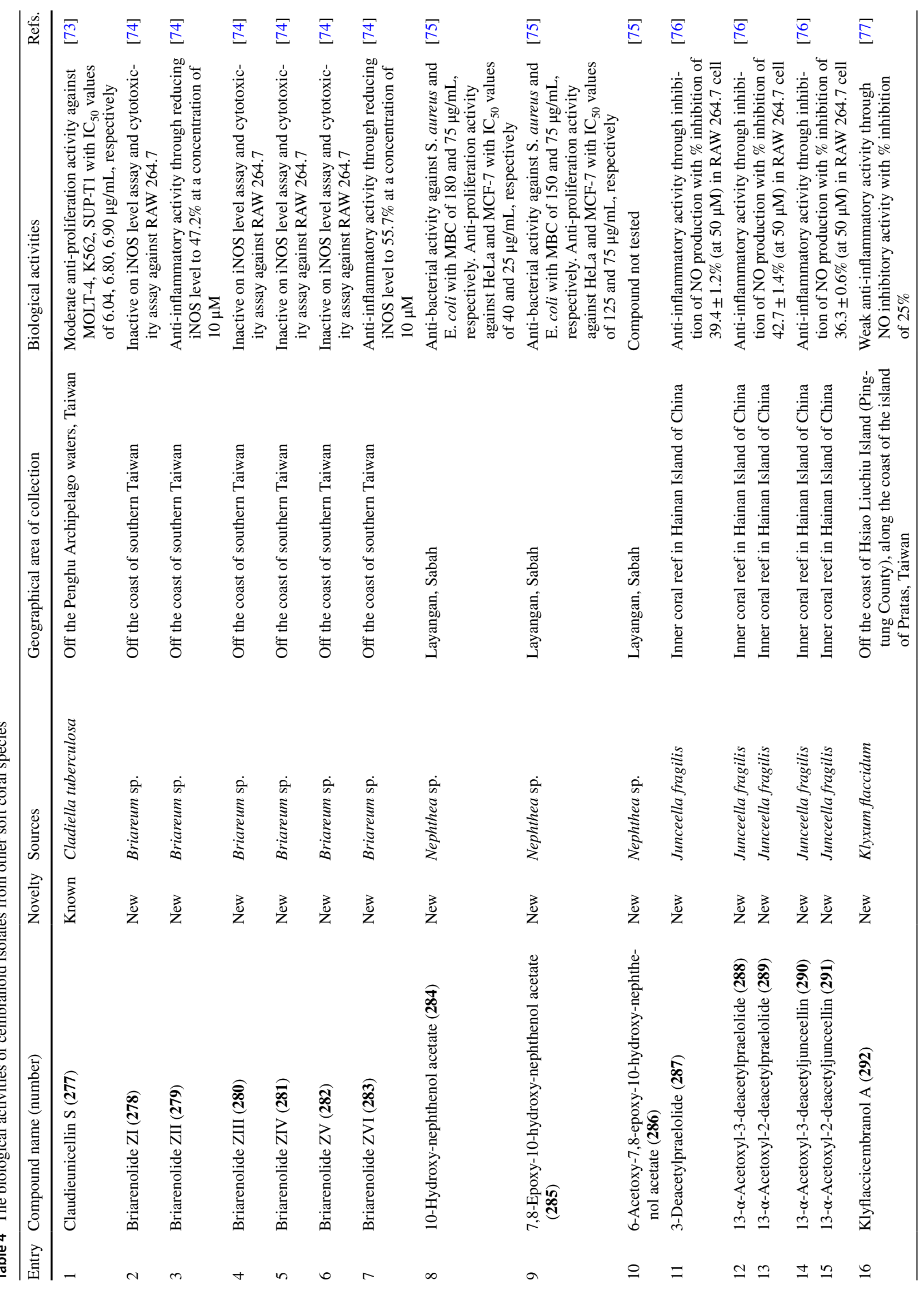




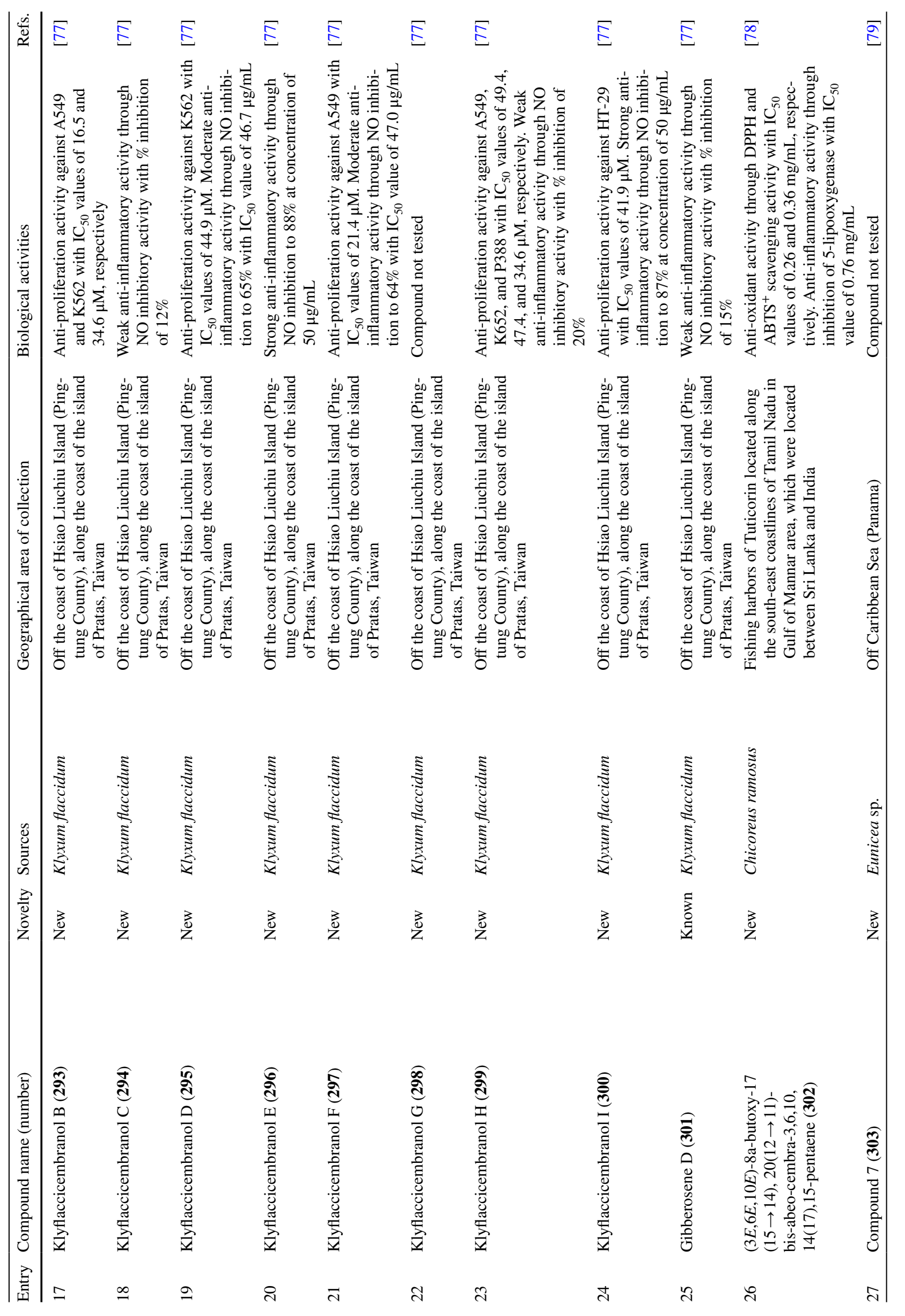



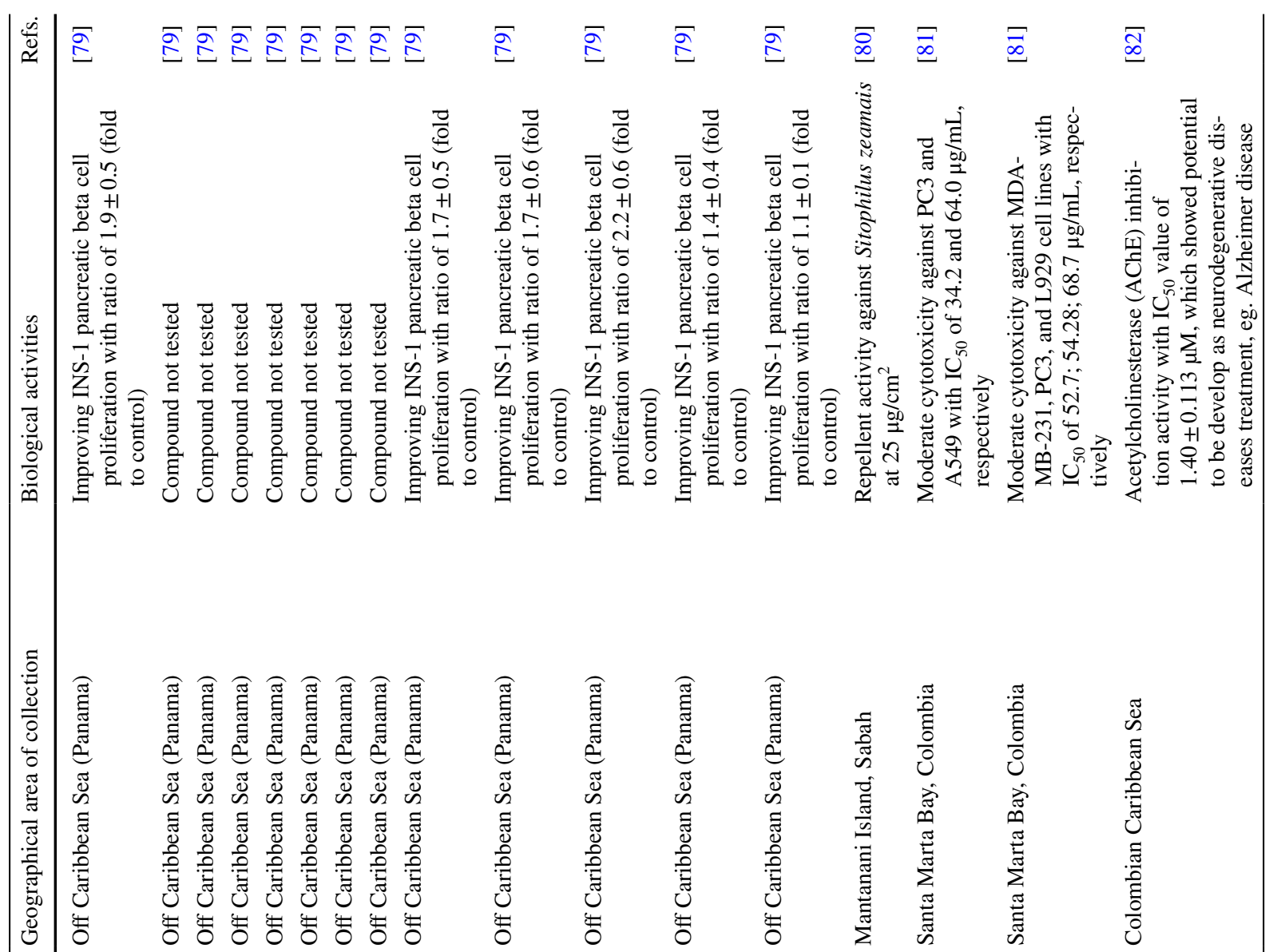

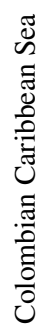

क के के के के के के के

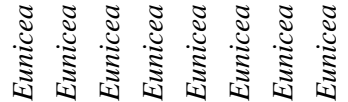

के

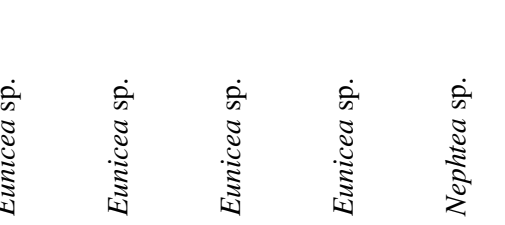

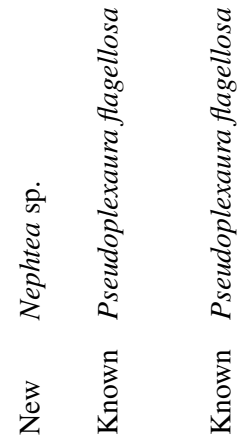

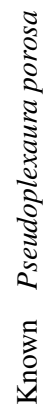

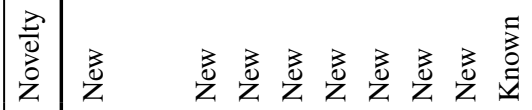

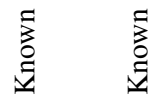

ป
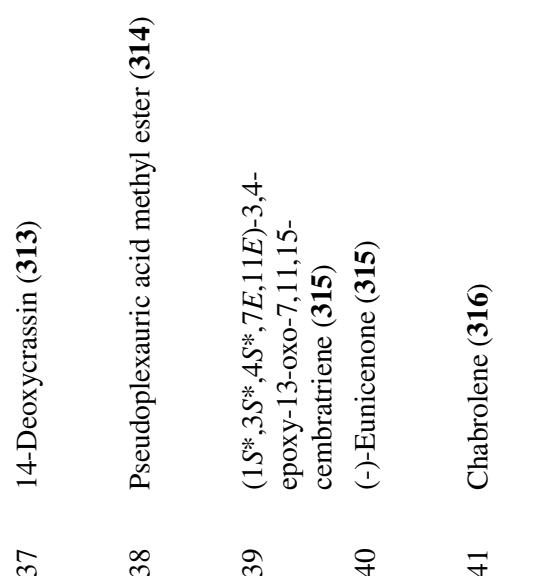

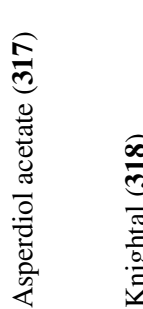

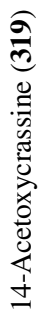

总

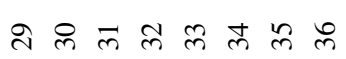

m

ले \&

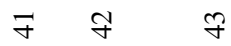

タ 


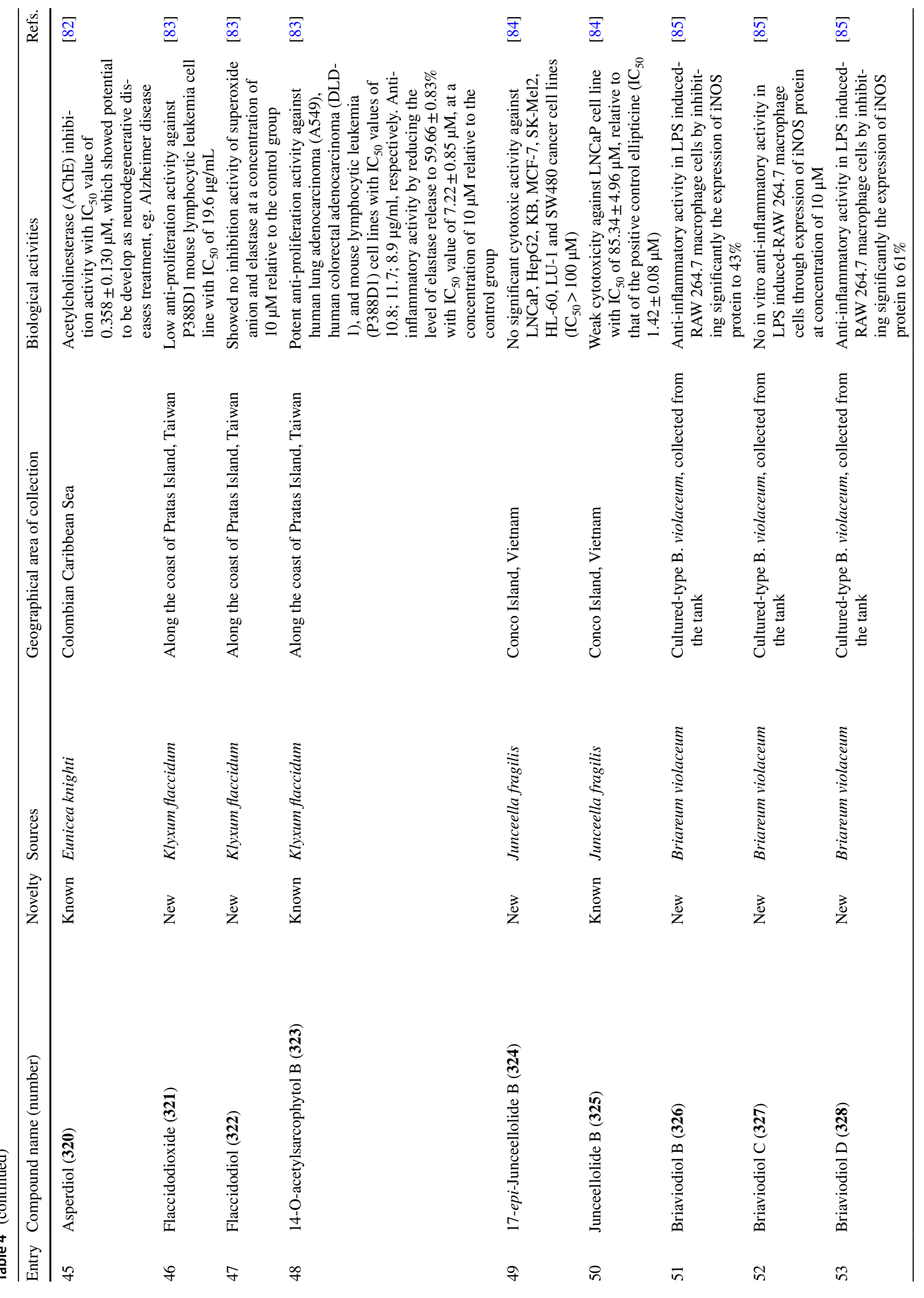




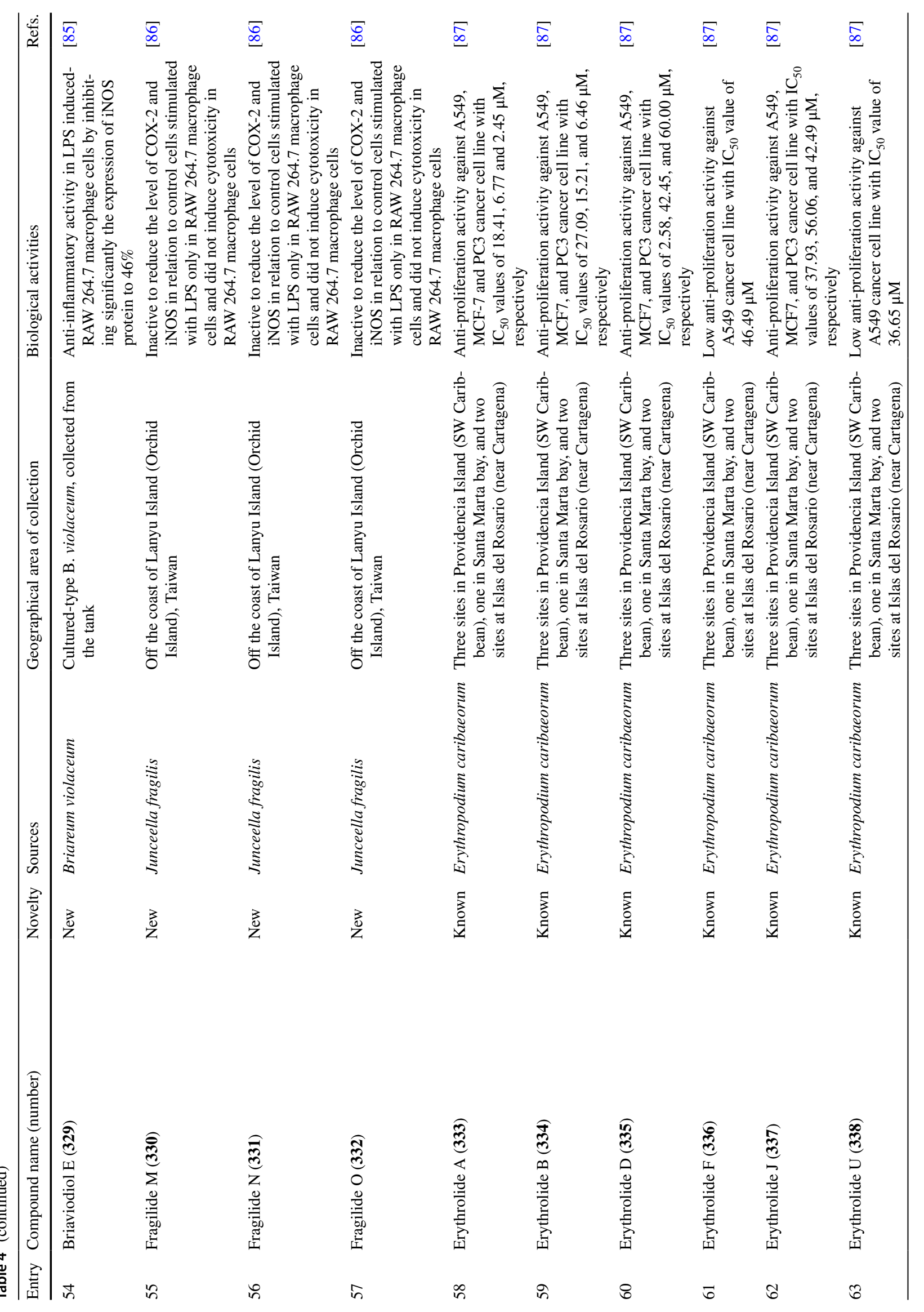




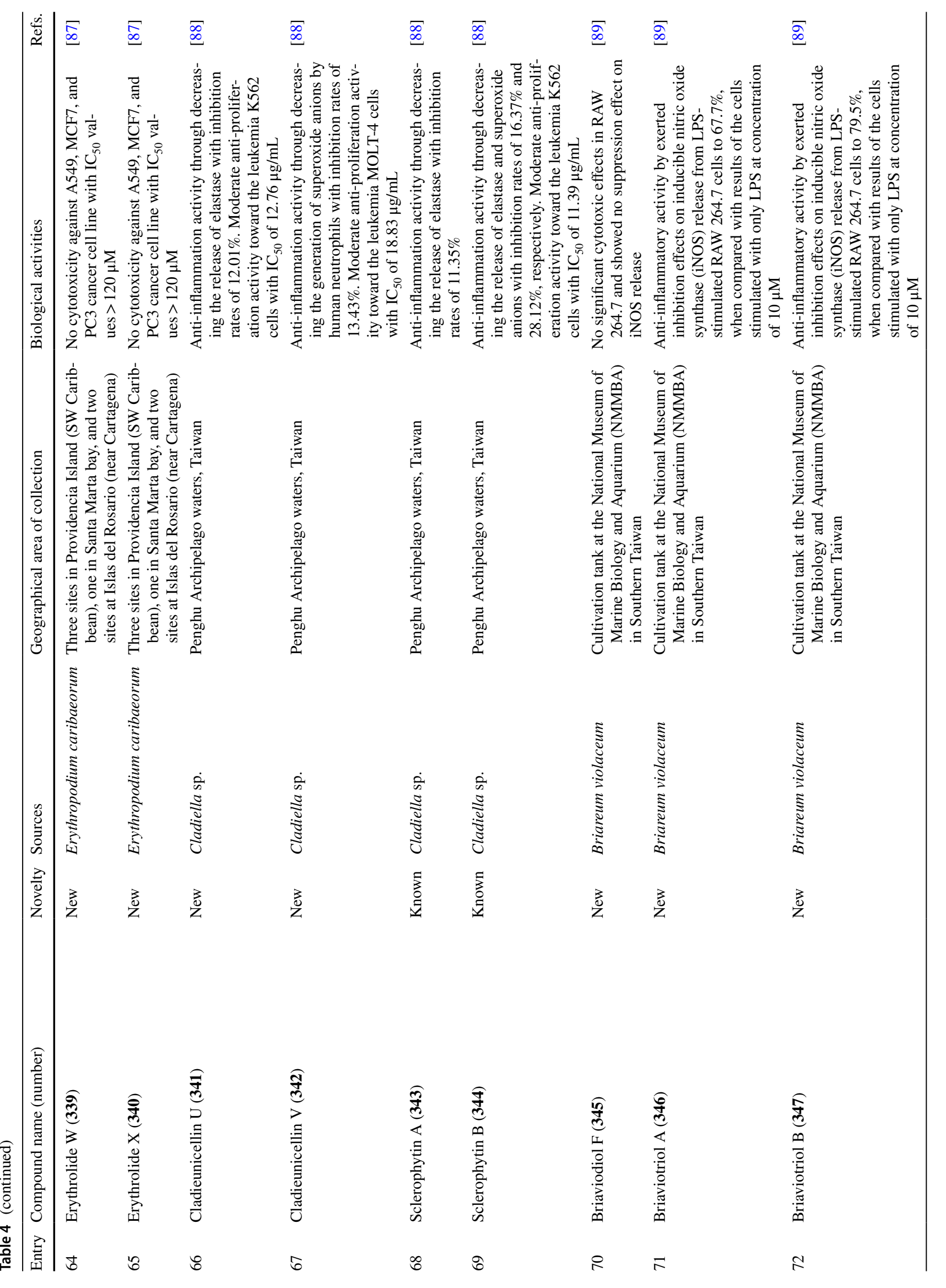




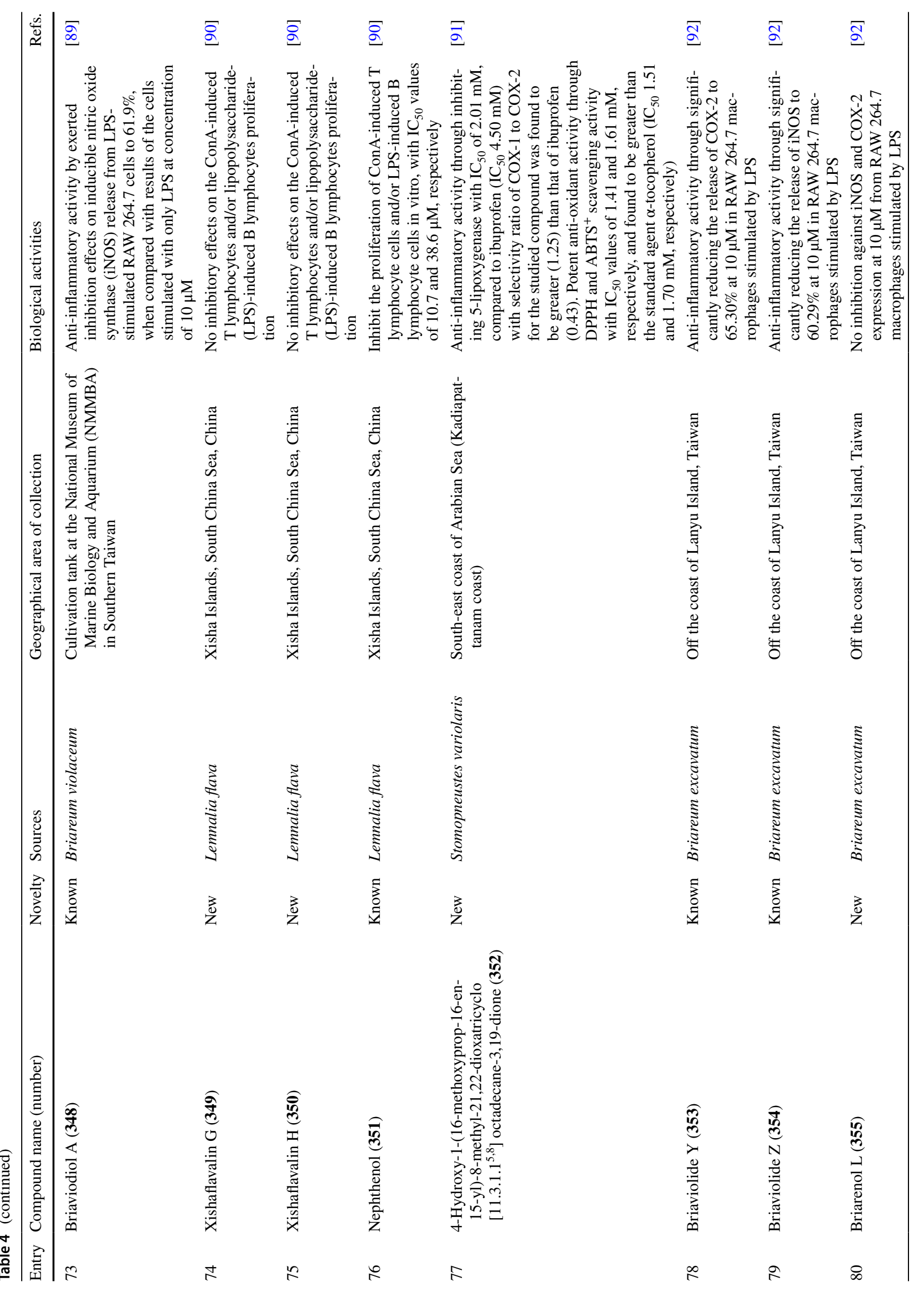




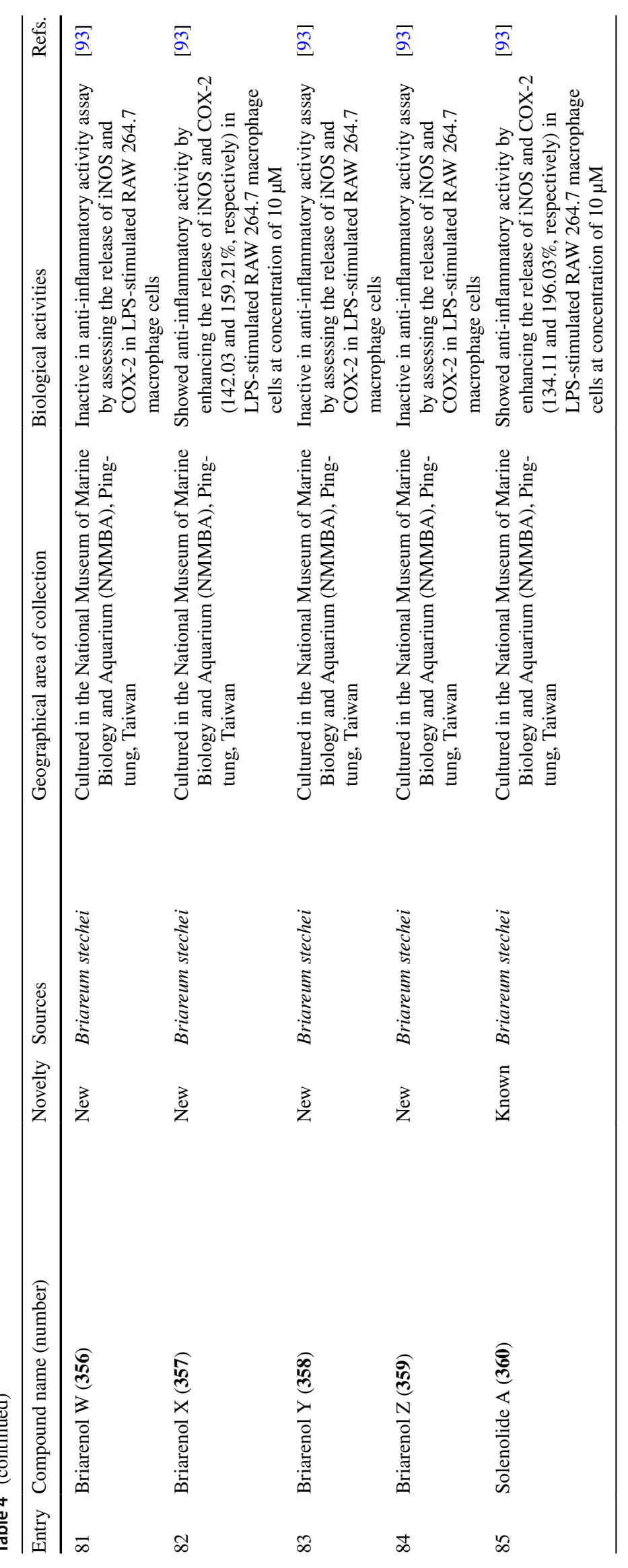




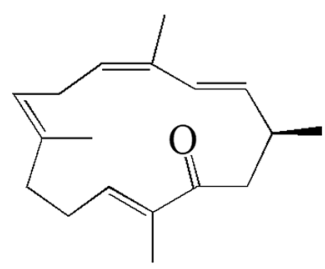

212
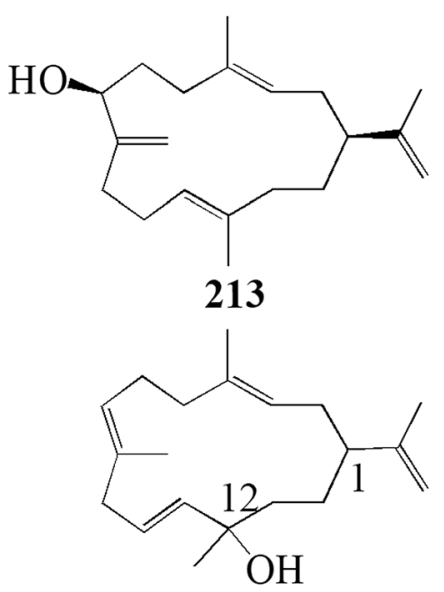

1S, 12S (214)

1S, 12R (215)

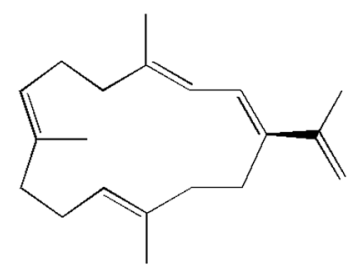

216
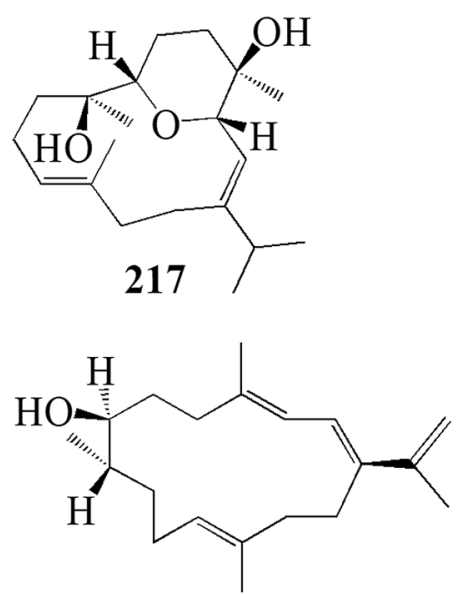

218

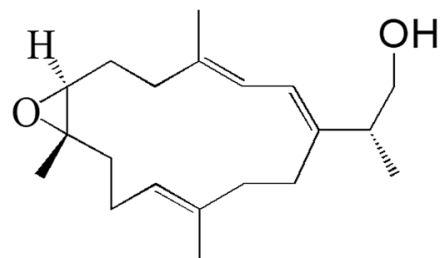

219

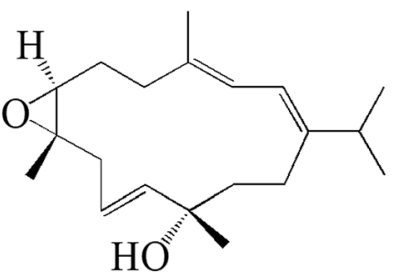

220

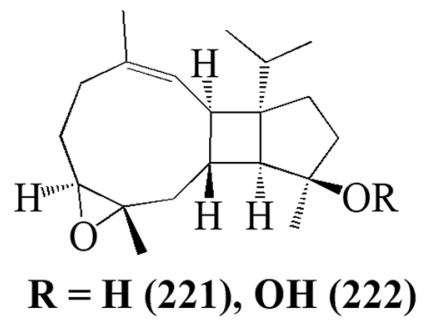

Fig. 9 Cembranoids isolated from Sinularia crassa (212-216) and Sinularia humilis (217-222)

sp. collected from Sabah, Malaysia. 10-hydroxy-nephthenol acetate 284 and 7,8-epoxy-10-hydroxy-nephthenol acetate $\mathbf{2 8 5}$ were found to be biologically active, whilst 6-acetoxy-7,8-epoxy-10-hydroxy-nephthenol acetate $\mathbf{2 8 6}$ was not tested yet for its biological activity [75]. Junceella fragilis from Hainan Island, China contained five new briarane diterpenoids named 3-deacetylpraelolide 287, 13- $\alpha$-acetoxyl-3-deacetylpraelolide $\mathbf{2 8 8}, 13-\alpha$-acetoxyl-2deacetylpraelolide 289, 13- $\alpha$-acetoxyl-3-deacetyljunceellin 290, and 13- $\alpha$-acetoxyl-2-deacetyljunceellin 291 [76]. Several cembranoids were also isolated from Klyxum flaccidum originated from Hsiao Liuchiu Island, Taiwan, named klyflaccicembranols A-I 292-300 and gibberosene D 301. Klyflaccicembranol G 298 was the only compound that has not been tested for its biological activities [77].

A novel cembrane has been isolated from Chicoreus ramosus collected in fishing harbors between Sri Lanka and India, namely (3E, 6E, 10E)-8a-butoxy-17(15 $\rightarrow 14$ ), $20(12 \rightarrow 11)$-bis-abeo-cembra-3,6,10,14(17),15-pentaene 302 [78]. Meanwhile, nine new compounds were isolated from Eunica sp. collected from Caribbean Sea, namely compound 7-15 303-311. Among these compounds, 304 was the only one showing biological activities, whilst the other compounds were not tested yet. Moreover, five known cembranoids were also isolated this species, namely euniolide 312, 14-deoxycrassin 313, pseudoplexauric acid methyl ester
314, $\left(1 S^{*}, 3 S^{*}, 4 S^{*}, 7 E, 11 E\right)$-3,4-epoxy-13-oxo-7,11,15-cembratriene 315, and (-)-eunicenone 316 [79].

The Bornean soft coral Nephtea sp. collected from Mantanani Island, Sabah, was found to produce new cembranoid norditerpene, i.e. chabrolene 317 [80]. Pseudoplexaura flagellosa collected from Colombia was reported to have two known cembrane diterpenes, namely asperdiol acetate 318 and knightal 319 [81]. Known compounds 14-acetoxycrassine $\mathbf{3 2 0}$ and asperdiol $\mathbf{3 2 1}$ were successfully obtained from Pseudoplexaura porosa and Eunicea knighti collected in the Caribbean sea, respectively[82]. In 2019, Tseng and co-workers reported cembranoids from Klyxum flaccidum collected from Pratas Island, Taiwan. This species contained two new compounds, which are flaccidodioxide $\mathbf{3 2 2}$ and flaccidodiol 323. The later compound was reported to possess no activities. Moreover, a known compound 14-O-acetylsarcophytol B $\mathbf{3 2 4}$ was also reported from the same species [83]. Junceella fragilis collected from Vietnam was reported to produce new briarane-type diterpenoids, 17-epijunceellolide B $\mathbf{3 2 5}$ and junceellolide B $\mathbf{3 2 6}$. While 325 did not possess any activities, the later showed new activities than before [84].

Aquacultured Briareum violaceum has been reported to contain four novel hydroperoxyfurancembranoids, namely briaviodiols B-E 327-330. One compound named briaviodiol C 328 did not possess any activity [85]. In 2019, 


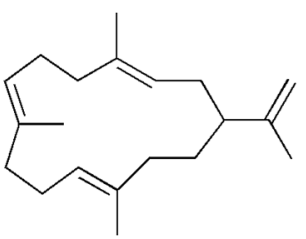

223<smiles>[Z10][C@@H](/C=C(/C)[C@@H](O)C/C=C\CC(=O)CC(C)C)CCC1[C@H](C)[C@H]1C</smiles>

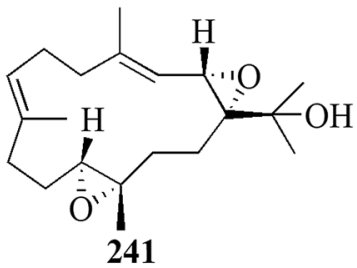

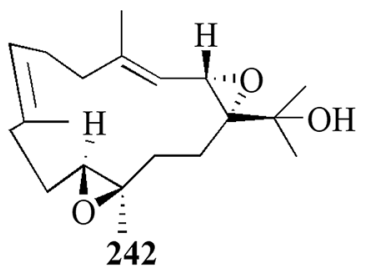

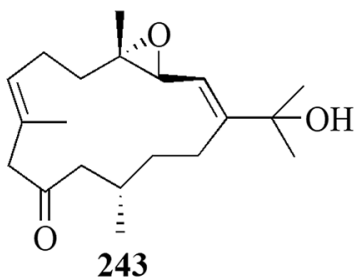

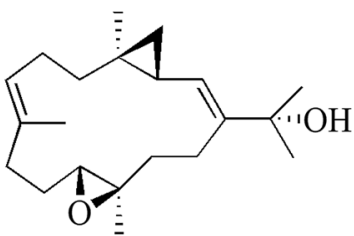

244
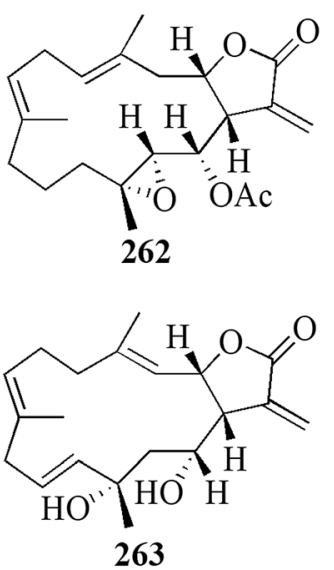<smiles>C=C(/C=C\CC/C(C)=C/[C@H]1OC(=O)C(=C)[C@H]1CC[C@](C)(O)/C=C/C)OC(C)C</smiles>

264

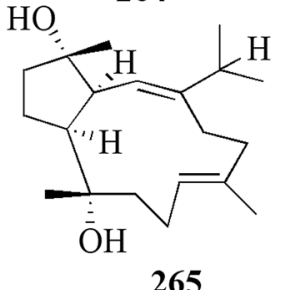

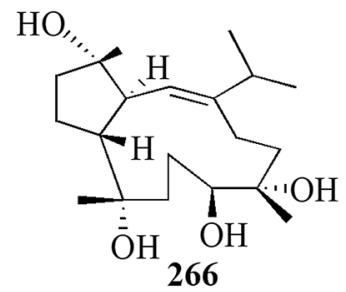

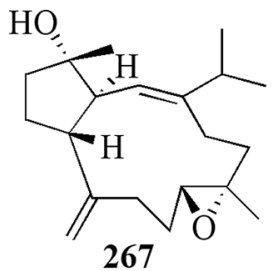

$\mathrm{HO}^{\prime}$
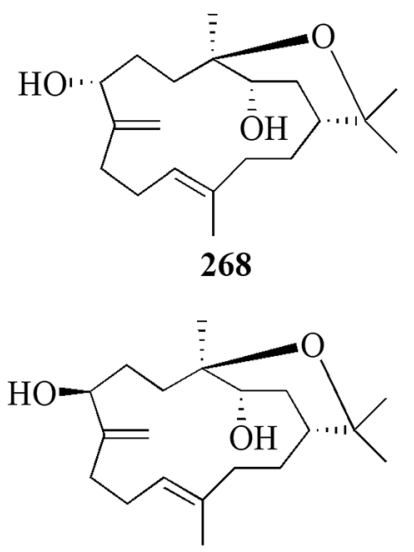

269

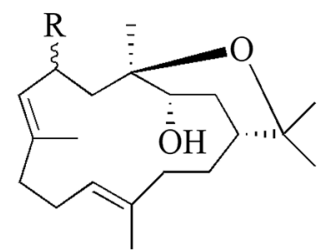

$\mathrm{R}=\beta-\mathrm{OH}(\mathbf{2 7 0}) ; \boldsymbol{\alpha - O H}(\mathbf{2 7 1})$

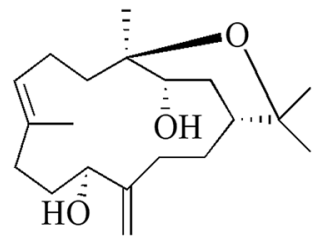

272
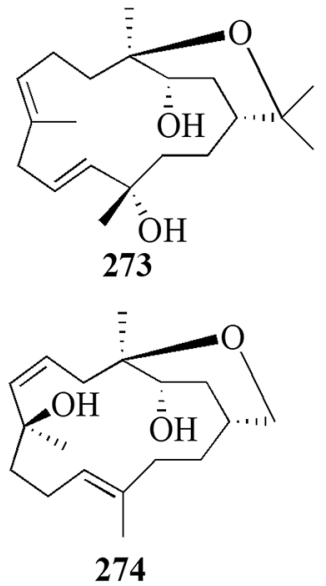<smiles>C=C1CCC=C(C)CC[C@]2(C)C[C@@H]1OC(C)(C)O2</smiles>

275

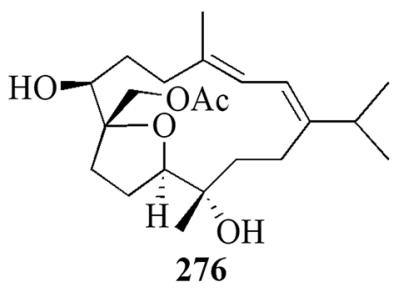

Fig. 10 Cembranoids reported from Lobophytum sp

three new-non active triacetoxybriaranes were isolated from Junceella fragilis in Lanyu Island, Taiwan, namely fragilides M-O 331-333 [86]. Eight chlorinated briarane diterpenoids were isolated from Erythropodium caribaeorum originated from Providencia Island, Caribbean. Among these, six known compounds, namely erythrolides A-B 334-335, erythrolide D 336, erythrolide F 337, erythrolide $\mathbf{J} \mathbf{3 3 8}$, and erythrolide $\mathrm{U339}$, were reported to have new activities. However, the new compounds erythrolides W-X 340-341 showed no biological activities [87]. Cladiella sp. from Taiwan contained two new eunicellin diterpenoids cladieunicellins U-V 342-343 and two known eunicellin diterpenoids sclerophytins A-B 344-345 [88].

Aquacultured Briareum violaceum from Southern Taiwan was found to yield three new furanocembranoids and one known furanocembranoid. The new-non active compound was briaviodiol F 346, while the two other new compounds named briaviotriols A-B 347-348 were biologically active. Briaviodiol A 349 was the only compound that had been isolated before [89]. Xishaflavalins G-H 350-351 were the new isolated cembrane from Chinese soft coral Lemnalia flava which did not show any activities, whereas new activities were reported from the known cembrane nephthenol 352 [90]. Stomopneustes variolaris from the Arabian Sea contained new cembrane named 4-hydroxy-1-(16-methoxyprop16-en-15-yl)-8-methyl-21,22-dioxatricyclo [11.3.1.1 ${ }^{5,8}$ ] octadecane-3,19-dione 353 [91]. Lastly, B. excavatum from Lanyu Island, Taiwan, contained two known briarane diterpenoids named briaviolides $\mathrm{Y}-\mathrm{Z}$ 354-355 and one new-non active briarane diterpenoid named briarenol L 356 [92]. 
<smiles>COC(=O)/C=C(\CC/C(=C/CC[C@@]1(C)O[C@@H]1C)C(=O)OC)C(=O)OC</smiles><smiles>[R16]C(=O)CCCC1=C(C)C(=O)OC1/C=C(\C)CC/C=C\CC</smiles>

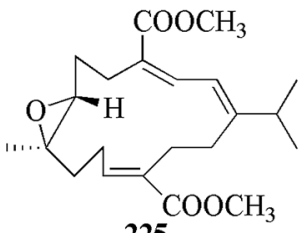<smiles>CC(=CCCC1(C)CO1)CCC1=C(C)C(=O)OC1C=C(C)CCC1(C)CO1</smiles>

234

225<smiles>COC(=O)[C@]12CCC(C(C)C)=C[C@H]1[C@]1(C(=O)OC)CCC(O)[C@@](C)(O)CC[C@@H]21</smiles><smiles>COC(=O)C(=CC=C(CC(C)C)C(=O)OC)CCC1CC[C@H]2CC[C@@H]1O2</smiles>

$\mathrm{COOCH}_{3}$

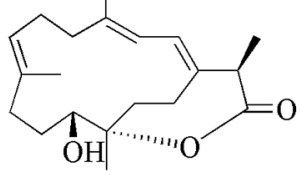

227

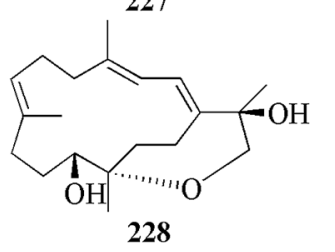<smiles>CC/C([12CH3])=C/CC[C@@]1(C)O[C@H]1CC/C(C)=C/[C@H]1OC(=O)C(C)(O)[C@@]1(C)O</smiles>

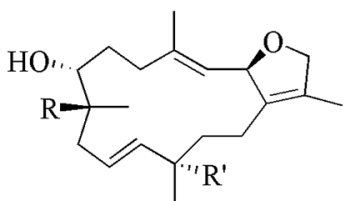

$\mathbf{R}=\mathbf{O C H}_{3}$ (230), OH (233)

$\mathbf{R}^{\prime}=$ OH (230), H (233)

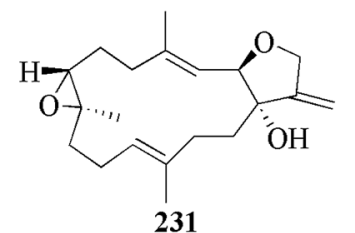

231

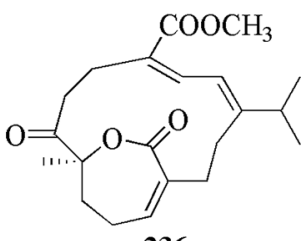

236

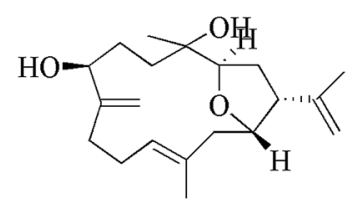

237

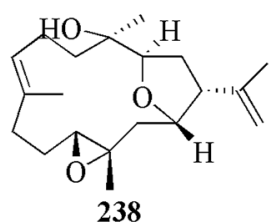<smiles>C=C(C)[C@H](C(O)/C=C(\C)CC/C=C\CC/C=C(\C)CCO)[C@@H](O)C/C(C)=C/C</smiles>

239

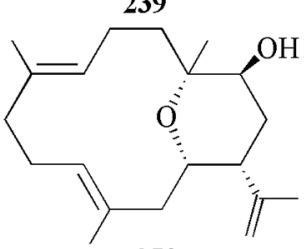

250

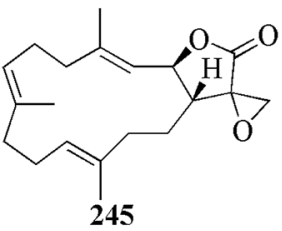<smiles>C=C(C)[C@H]1C[C@H](OC(C)(O)CC/C=C\CCC=C(C)C)[C@H](C)C1</smiles><smiles>C=C1C(=O)O[C@@H](C(=O)[C@H](C)CC/C=C\CC/C=C(\C)CC(C)C)[C@@H]1C(C)=O</smiles><smiles>C=C1C(=O)OC(CC2(C)CO2)C1C/C=C(\C)CC/C=C\CC1CO1</smiles>

254<smiles>[3H]/C(=C\CC/C1=C(\C)CCC2(C)OC2C[C@H]1CC1OC1C)CC/C=C\C</smiles><smiles>C=C1C(=O)OC(C(O)/C(C)=C/CCC2=CCCC3(C)OC3C2)[C@@H]1O</smiles>

$\mathrm{HOO}$

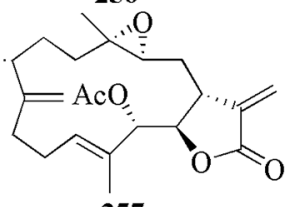

257<smiles>C=C(CO)[C@H](C/C=C(\C)CC/C=C\CCC=C(C)C)C(=O)C/C=C\C</smiles>

249<smiles>C=C(C)[C@H]1CC(O)C(C)(CC/C=C(/C)CCC=C(C)C)O[C@@H]1C/C=C\C</smiles>

258

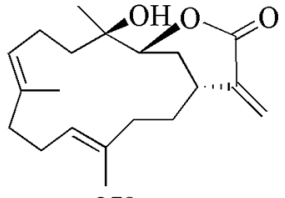

259<smiles>C=C(C(=O)O)C(CC/C([Al])=C/CC/C=C\CCC1(C)O[C@@H]1C)C(=O)O</smiles>

Oㅡㅁ

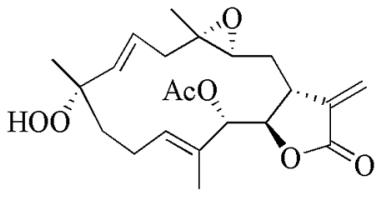<smiles>C=C(CO)[C@H]1CC/C(C)=C/CC/C=C\CC[C@]1(C)CC1O[C@@H]1C</smiles>

252

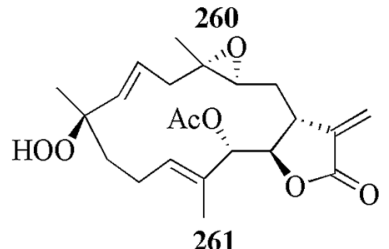

Fig. 11 Cembranoids reported from Lobophytum crassum 


\section{Biological Activities}

Cembranoids and their analogues have been reported to have various biological activities such as anti-cancer, anti-bacterial, anti-inflammation, anti-diabetic, neurological activity, anti-fouling, toxicity to brine shrimp, immunosuppressant, anti-Alzheimer's, anti-oxidant, repellent activity against Sitophilus zeamais, and acetylcholinesterase (AChE) inhibition activity. The reported total numbers of cembranoid compounds from genera Sarcophyton, Sinularia, Lobophytum, and other species that were successfully identified were $139,42,47$, and 80 , respectively. Among them, 221 were newly isolated compounds, and the other 87 compounds were previously known with newly discovered activities. The remaining 34 new compounds have not been tested for their biological activities.

\subsection{Anti-bacterial}

Compound 1 showed antibacterial activity against Staphylococcus aureus, with minimum bactericidal concentration (MBC) and minimum inhibitory concentration (MIC) values of 75 and $25 \mu \mathrm{M}$, respectively [17]. Compound 2 isolated from Sarcophyton trocheliophorum also possessed moderate antibacterial activity against Bacillus subtilis, Staphylococcus aureus, and Vibrio cholerae with MIC values of 125,100 and $125 \mathrm{mg} / \mathrm{mL}$, respectively, but it did not have activity against Escherichia coli [18]. Compound $\mathbf{8}$, exhibited antibacterial activity against several bacteria, viz. Acinetobacter baumannii $(\mathrm{MIC}=4.2 \mu \mathrm{M})$, Escherichia coli $(\mathrm{MIC}=6.0 \mu \mathrm{M})$, Klebsiella pneumoniae $(\mathrm{MIC}=5.8 \mu \mathrm{M})$, Pseudomonas aeruginosa $(\mathrm{MIC}=5.2 \mu \mathrm{M})$, Staphylococcus aureus $(\mathrm{MIC}=4.0 \mu \mathrm{M})$, Staphylococcus epidermidis $(\mathrm{MIC}=5.7 \mu \mathrm{M})$, and Streptococcus pneumoniae $(\mathrm{MIC}=6.0 \mu \mathrm{M})$. While, 9 and $\mathbf{1 0}$, which were also tested against the bacteria mentioned above, showed weak antibacterial activity. Compound 9 was reported to have inhibition zones of $7,8,7$, and $7 \mathrm{~mm}$ zones of 11, 11, and $6 \mathrm{~mm}$ against Klebsiella pneumonia, Staphylococcus aureus, and Staphylococcus epidermidis, respectively [21]. The compound from Staphylococcus trocheliophorum, 84, exerted moderate antibacterial activity against Staphylococcus aureus with MIC value of $250 \mu \mathrm{M}$ [36]. Additionally, 85 exhibited anti-fungal activity towards Ochroconis humicola and Haliphthoros milfordensis with MIC value of $6.25 \mu \mathrm{g} / \mathrm{mL}$ [37].

Compound 223 isolated from Lobophytum sp. showed moderate anti-bacterial activity against Acinetobacter sp., Escherichia coli, Klebsiella pneumonia, Pseudomona aeruginosa, Staphylococcus aureus, Staphylococcus epidermidis, and Streptococcus pneumonia. It had inhibition zone diameters of 14,13,13,13,11,11, $11 \mathrm{~mm}$, respectively and MIC value of $30 \mu \mathrm{g} / \mathrm{mL}$ against those bacteria [63]. The Okinawan Lobophytum sp. produced five cembranoid compounds (240-244) that exhibited antibacterial activity against Staphylococcus aureus and Eschericia coli. At a concentration of $25 \mu \mathrm{g}$ compound 199-203 had an inhibition zone of $10,9,9,10,10 \mathrm{~mm}$, respectively against Staphylococcus aureus and 10, 10, 10, 12, $15 \mathrm{~mm}$, respectively against Escherichia coli [66]. Furthermore, cembranoids isolated from Nephthea sp., 284 and 285, exerted anti-bacterial activity against Staphylococcus aureus with MBC of 180 and $150 \mu \mathrm{g} / \mathrm{mL}$, respectively and Eschericia coli with MBC of 75 and $75 \mu \mathrm{g} / \mathrm{mL}$, respectively [75].

\subsection{Anti-cancer}

New compounds 24, 25 and $\mathbf{2 6}$ isolated from Sarcophyton ehrenbergi showed low to moderate anti-proliferation activity against A549 human lung carcinoma cells with inhibition concentration $50\left(\mathrm{IC}_{50}\right)$ values of $50.1,76.4$, and $50.8 \mu \mathrm{M}$, respectively, but inactive towards Caco-2 human colorectal adenocarcinoma cells. Compounds $\mathbf{2 4}$ and $\mathbf{2 6}$ also exhibited low to moderate cytotoxicity to HepG2 human liver carcinoma cells with $\mathrm{IC}_{50}$ values of 98.6 and $53.8 \mu \mathrm{M}$ [17]. In addition, the known compounds 27-31 isolated from Sarcophyton glaucom also exerted moderate to potent activity against HepG2. Compound $\mathbf{2 7}$ and $\mathbf{2 8}$ were tested together and exerted effective concentration $50\left(\mathrm{EC}_{50}\right)$ value of $11.32 \mu \mathrm{g} / \mathrm{mL}$, while compound $\mathbf{2 9}-\mathbf{3 1}$ possessed $\mathrm{EC}_{50}$ values of $17.84 ; 9.97$; and $10.32 \mu \mathrm{g} / \mathrm{mL}$, respectively [26]. Another study reported anti-cancer activity against MCF-7 human breast cancer cells from compounds $\mathbf{3 0}$ and $\mathbf{3 1}$ with $\mathrm{IC}_{50}$ values of 24.97 and $22.39 \mu \mathrm{g} / \mathrm{mL}$, respectively [32].

Compounds 42-47 also showed cytotoxic activity towards MCF-7, with growth inhibition $50\left(\mathrm{GI}_{50}\right)$ values of 18.13 ; $12.22 ; 24.2 ; 22.27 ; 18.88$; and $20.041 \mathrm{ppm}$, respectively [29]. Compound 62 extracted from Sarcophyton mililatensis was reported to have strong cytotoxicity towards HL-60 human leukemia cells and $\mathrm{A} 549$ cells, with $\mathrm{IC}_{50}$ values of $0.78 \mu \mathrm{mol} / \mathrm{mL}$ and $1.26 \mu \mathrm{mol} / \mathrm{mL}$, respectively [31]. New compounds 92-96 showed cytotoxicity towards MCF-7 cells with $\mathrm{IC}_{50}$ values of $23.84 ; 26.22 ; 26.81 ; 25.28$; and $27.2 \mu \mathrm{g} /$ $\mathrm{mL}$, respectively [32]. New potent anti-cancer activity from known compounds 103, 104 and 105 isolated from Sarcophyton ehrenbergi was reported against A549 cells, with inhibition concentration $25\left(\mathrm{IC}_{25}\right)$ values of $23.3,27.3$, and $25.4 \mu \mathrm{M}$, respectively. However, they were not active against Caco- 2 cells. Additionally, $\mathbf{1 0 4}$ and $\mathbf{1 0 5}$ exhibited weaker activity against $\mathrm{HepG} 2$ cells with $\mathrm{IC}_{25}$ values of 22.6 and $31.8 \mu \mathrm{M}$, respectively [40]. Finally, from genus Sarcophyton, 139, a known compound isolated from Sarcophyton glaucum exhibited anti-proliferation activity against HEK293 
human embryonic kidney cells with lethal dose $50\left(\mathrm{LD}_{50}\right)$ of $123.5 \mathrm{mM}$ [44].

Compound 141, 145-147 isolated from Sarcophyton digitatum showed anti-cancer activity towards various cancer cell line. Compound 141 showed cytotoxicity against MCF-7 and MDA-MB-231 with $\mathrm{IC}_{50}$ of $9.6 \pm 3.0$ and $14.8 \pm 4.0 \mu \mathrm{g} /$ $\mathrm{mL}$, respectively. Moreover, 145 showed cytotoxicity towards MCF-7, HepG2, and HeLa with $\mathrm{IC}_{50}$ values of $10.1 \pm 3.3 ; 14.9 \pm 3.5$; and $17.1 \pm 4.5 \mu \mathrm{g} / \mathrm{mL}$, respectively. In addition, 146 exhibited cytotoxicity towards MCF-7, MDAMB-231, and HepG2 with $\mathrm{IC}_{50}$ value of $9.4 \pm 3.0 ; 17.8 \pm 4.5$; $14.9 \pm 4.2 \mu \mathrm{g} / \mathrm{mL}$, respectively. Lastly, 147 showed cytotoxicity towards MCF-7 with an $\mathrm{IC}_{50}$ value of $10.9 \pm 4.3 \mu \mathrm{g} /$ $\mathrm{mL}$ [45].

Another study reported that cembranoid isolated from Sarcophyton tenuispiculatum also possessed anti-cancer activity including compound 148, 151-155. Compound 148, $151-155$ showed cytotoxicity against MCF-7 with $\mathrm{IC}_{50}$ value of $34.3 \pm 3.7 ; 37.6 \pm 4.2 ; 33.3 \pm 3.5 ; 30.1 \pm 3.1 ; 24.3 \pm 3.0$; $27.2 \pm 4.0 \mu \mathrm{m}$, respectively. Whilst compound $151-152$, 154-155 showed cytotoxicity against HepG2 with $\mathrm{IC}_{50}$ value of $35.2 \pm 4.4 ; 28.6 \pm 3.4 ; 34.5 \pm 4.2$ and $36.4 \pm 5.3 \mu \mathrm{m}$, respectively. Furthermore, compound 153 showed cytotoxicity towards MDA-MB-231 cell line with an $\mathrm{IC}_{50}$ value of $38.6 \pm 5.0 \mu \mathrm{m}[46]$.

Several compounds from the genus Sinularia were also reported to have anti-cancer activity. Compound $\mathbf{1 7 2}$ from Sinularia erecta showed anti-proliferation activity against $\mathrm{K} 562$ human leukimia cell line with an $\mathrm{IC}_{50}$ value of $9.2 \mu \mathrm{M}$ [49]. Compound 178 from Sinularia compacta showed anti-proliferation activity against HCT-116 human colorectal carcinoma cell and $\mathrm{A} 549$, with $\mathrm{IC}_{50}$ values of 10.1 and $14.7 \mu \mathrm{M}$, respectively [53]. The cembranoid compound, 178, isolated from Sinularia sp. found in Yongxing Island, South China Sea had anti-cancer activity was towards HeLa human cervical cancer and HCT-116 with $\mathrm{IC}_{50}$ values of 11.6 and $33.3 \mu \mathrm{M}$, respectively [54].

In 2018, Tsai et al. isolated $\mathbf{1 8 1}$ from aquacultured Sinularia sandensis. The compound exerted a concentrationdependent anti-proliferative effect on NCI-N87 human gastric carcinoma cells and promoted apoptosis induction. The anti-proliferation activity was associated with the release of cytochrome $\mathrm{c}$ from mitochondria, activation of pro-apoptotic proteins, e.g. cysteine-aspartic proteases(caspase)-3/-9, Bcl2-associated $\mathrm{X}$ protein (Bax) and Bcl-2-associated agonist of cell death (Bad), and inhibition of the anti-apoptotic proteins B-cell lymphoma 2 (Bcl-2), B-cell lymphoma-extra large (Bcl-xL), and myeloid cell leukemia 1 (Mcl-1). This compound also triggered endoplasmic reticulum (ER) stress, leading to activation of the PERK/elF2 $\alpha / \mathrm{ATF} 4 / \mathrm{CHOP}$ apoptotic pathway. Further, $\mathbf{1 8 1}$ also initiated autophagy in NCIN87 cells and induced the expression of autophagy-related proteins, including Autophagy related (Atg)3, Atg5, Atg7,
Atg12, microtubule-associated protein light chain (LC)3-I, and LC3-II [55].

Compounds 182 and 183 isolated from Sinularia sp. found in Sabah, Malaysia possessed anti-proliferation activity against HL-60 cancer cell line through apoptosis mechanism that involved the up-regulation of Bax, the downregulation of Bcl-xL, and the activation of caspase-3 [56]. Wu et al. isolated 7 cembranoids, 184-190, from Sinularia flexibilis whereas four of them (187-190) exhibited anti-proliferation activity. Compound $\mathbf{1 8 7}$ showed anti-proliferation activity against P388 mouse leukimia cells, K562, HT-29 human colon cancer cell lines, with $\mathrm{IC}_{50}$ values of 9.3, 23.4, and $15.9 \mu \mathrm{M}$, respectively. Compound $\mathbf{1 8 8}$ exhibited antiproliferation activity against P388, K562, HT-29 cancer cell lines, with $\mathrm{IC}_{50}$ values of $6.9,12.2$, and $9.6 \mu \mathrm{M}$, respectively. Compound $\mathbf{1 8 9}$ showed anti-proliferation activity against P388 and K562 cancer cell lines, with $\mathrm{IC}_{50}$ values of 16.0 and $26.7 \mu \mathrm{M}$, respectively. Compound 190 exerted anti-proliferation activity against K562 and HT-29 cancer cell lines, with $\mathrm{IC}_{50}$ values of 21.7 and $27.1 \mu \mathrm{M}$, respectively [57]. Cembranoid 187 isolated from Sinularia flexibilis collected in Hainan exerted broad anti-proliferation activity against A549, HT-29, SNU-398 human hepatocellular carcinoma, and Capan-1 human pancreatic ductal adenocarcinoma cell line, with $\mathrm{IC}_{50}$ values of $27.4,22.7,8.9$, and $9.4 \mu \mathrm{M}$, respectively [58]. $\mathbf{1 8 8}$ and $\mathbf{1 9 0}$ isolated from the same species in Hainan, China, showed moderate anti-proliferation activities against HT-29, SNU-398, and Capan-1, with $\mathrm{IC}_{50}$ values of 32.6; 24.9; $28.7 \mu \mathrm{M}$ and 33.6; 24.7; $26.1 \mu \mathrm{M}$, respectively [58]. Compound 191 isolated from aquaculture Sinularia flexibilis in Taiwan exerted anti-oral cancer activity by inducing oxidative stress-mediated cell death pathways through suppressing colony formation, inducing apoptosis and cell cycle arrest, as well as inducing reactive oxygen species (ROS) as observed in three in vitro cultured human oral squamous cell carcinoma (OSCC) models (Ca9.22, SCC9 and HSC-3 cell lines) [59].

Compound 223 from Lobophytum sp. exerted significant anti-tumor activity against Ehrlich ascites carcinoma cells with $\mathrm{LD}_{50}$ of $50 \mu \mathrm{g} / \mathrm{mL}$ [63]. Roy et al. isolated 240-242 from the Okinawan soft coral Lobophytum sp. These compounds showed mild cytotoxicity against HCT-116, with $\mathrm{IC}_{50}$ values of $135.37,177.11$, and $153.11 \mu \mathrm{M}$, respectively [66]. Out of the twelve new cembranoids isolated from aquacultured Lobophytum crassum collected from the coast of Pingtung, Taiwan, 250-261, ten showed anti-proliferation activitiy [67]. Compound 211 had $\mathrm{IC}_{50}$ of $35.8 \mu \mathrm{M}$ against SUP-T1 human T-cell lymphoblastic lymphoma cell, compound 212 had activity against K562, MOLT-4 human acute $\mathrm{T}$ lymphoblastic leukaemia A, SUP-T1, with $\mathrm{IC}_{50}$ values of $16.3,12.3$, and $4.6 \mu \mathrm{M}$, respectively, while compounds 254-261 was active against K562, Molt 4, U937 human myeloid leukaemia cell line, and SUP-T1. The $\mathrm{IC}_{50}$ 
of compounds 254-261 against K562 were 11.3, 18.1, 3.3, $15.3,4.5,3.3,12.3$, and $13.0 \mu \mathrm{M}$, respectively; against MOLT-4 were 6.2, 8.4, 1.2, 11.6, 2.9, 2.3, 4.8, and 7.0 $\mu \mathrm{M}$, respectively; against U937 were 15.8, 4.4, 7.1, 32.0, 7.0, 5.2, 10.9 , and $23.3 \mu \mathrm{M}$, respectively; and against SUP-T1 were $5.2,8.3,1.5,10.2,4.5,6.2,6.1$, and $6.6 \mu \mathrm{M}$, respectively [67].

Compound 256 from aquacultured Lobophytum crassum showed cytotoxic activity against $\mathrm{Ca} 9-22$ human oral cancer cells through ROS generation and the suppression of the anti-oxidant enzyme activity. The apoptotic effect was found to be mediated through the interruption of the Keap1/ Nrf2/p62/SQSTM1 pathway. It increased the expression of apoptosis and DNA damage-related proteins in a concentration and time-dependent manner. It also exerted potent antitumor effect against oral cancer cells, as demonstrated by the in vivo xenograft animal model. This compound reduced the tumor volume by $55.29 \%$ and tumor weight by $90.33 \%$ [68]. In 2019, Roy et al., isolated three cembranoids, 262-264, from Okinawa, Japan, which showed anti-proliferation activity against various cancer cell lines. Compound $\mathbf{2 6 2}$ showed moderate anti-proliferation activity against HeLa, A459, B16-F10 mouse skin melanoma, and RAW 264.7 mouse macrophage cells, with $\mathrm{IC}_{50}$ of $7.81,9.30,10.83$, and $5.99 \mu \mathrm{M}$, respectively. Compound 263 exerted low anti-proliferation activity against HeLa, A459, and RAW 264.7 cells, with $\mathrm{IC}_{50}$ of $49.33,54.09$, and $43.74 \mu \mathrm{M}$, respectively. Compound 264 possessed low anti-proliferation activity against RAW 264.7 cells, with $\mathrm{IC}_{50}$ of $45.22 \mu \mathrm{M}$ [69].

Lobophytum sp. collected from Xisha Island contained two compounds that exhibited anti-cancer activity. Compound 270 showed moderate cytotoxicity against SNU398 with an $\mathrm{IC}_{50}$ value of $42.54 \pm 6.26 \mu \mathrm{M}$. Besides, 271 exhibited anti-cancer activity towards various cancer cell line including HT-29, Capan-1, A549, and SNU-398 with $\mathrm{IC}_{50}$ values of $4.52 \pm 0.82 ; 6.62 \pm 4.02 ; 5.17 \pm 0.86$; $6.15 \pm 2.28 \mu \mathrm{M}$, respectively [72]. Compound 277 isolated from Cladiella tuberculosa possessed moderate anti-proliferation activity against MOLT-4, K562, SUP-T1, with $\mathrm{IC}_{50}$ values of $6.04,6.80,6.90 \mu \mathrm{g} / \mathrm{mL}$, respectively [73]. In 2016, Ishii et al., isolated $\mathbf{2 8 4}$ and $\mathbf{2 8 5}$ from the Bornean soft coral Nephthea sp. They possessed anti-proliferation activity against $\mathrm{HeLa}$ with $\mathrm{IC}_{50}$ values of 40 and $125 \mu \mathrm{g} / \mathrm{mL}$, respectively, and against MCF-7 with $\mathrm{IC}_{50}$ values of 25 and $75 \mu \mathrm{g} / \mathrm{mL}$, respectively [75]. In 2017, Ahmed et al., isolated 293 which showed anti-proliferation activity against A549 and $\mathrm{K} 562$ with $\mathrm{IC}_{50}$ values of 16.5 and $34.6 \mu \mathrm{M}$, respectively [77]. Four new compounds, namely 295, 296, 299 and 300, isolated from Klyxum flaccidum exerted anti-cancer activity towards various cancer cell lines. 295 possessed anti-proliferation activity against K562 with $\mathrm{IC}_{50}$ of $44.9 \mu \mathrm{M} .297$ showed anti-proliferation activity against A549 with $\mathrm{IC}_{50}$ of $21.4 \mu \mathrm{M} .298$ exerted anti-proliferation activity against
A549, K652, and P388 with $\mathrm{IC}_{50}$ values of 49.4, 47.4, and $34.6 \mu \mathrm{M}$, respectively. $\mathbf{3 0 0}$ displayed anti-proliferation activity against HT-29 with $\mathrm{IC}_{50}$ values of $41.9 \mu \mathrm{M}$ [77]. Two known compounds, 317 and 318 were isolated from Colombian Pseudoplexaura flagellosa. 317 showed moderate cytotoxicity against PC3 human prostate cancer cell line and A549 with $\mathrm{IC}_{50}$ of 34.2 and $64.0 \mu \mathrm{g} / \mathrm{mL}$, respectively. Further, 318 exerted moderate cytotoxicity against MDAMB-231 human breast cancer cell, PC3, and L929 mouse fibroblast cell lines with $\mathrm{IC}_{50}$ of $52.7,54.28$ and $68.7 \mu \mathrm{g} /$ $\mathrm{mL}$, respectively [81]. Tseng et al. (2019) isolated 321 and 323 from Taiwanese Klyxum flaccidum which showed anticancer activity. 321 displayed low anti-proliferation activity against the P388D1 mouse lymphocytic leukemia cell line with $\mathrm{IC}_{50}$ of $19.6 \mu \mathrm{g} / \mathrm{mL}$, while 323 showed a broad range of anti-cancer activities against A549, DLD-1 human colorectal adenocarcinoma, and P388D1 cell lines with $\mathrm{IC}_{50}$ values of $10.8,11.7$ and $8.9 \mu \mathrm{g} / \mathrm{ml}$, respectively [83]. The known compound, 325, isolated from Vietnamese Junceella fragilis showed weak cytotoxicity against the LNCaP human prostate adenocarcinoma cells with $\mathrm{IC}_{50}$ of $85.34 \mu \mathrm{M}$, as compared with that of the positive control ellipticine $\left(\mathrm{IC}_{50}\right.$ of $1.42 \mu \mathrm{M})$ [84].

In 2019, Molina et al. isolated six novel cembranoids (333-338) which possessed anti-cancer activity towards various cancer cell lines. 333, 334, 335 and 337 showed cytotoxicity against A549, MCF-7 and PC3 cancer cell lines. 333 possessed anti-tumor activity against A549, MCF-7 and PC3 with $\mathrm{IC}_{50}$ values of $18.41,6.77$ and $2.45 \mu \mathrm{M}$, respectively. 334 exerted anti-proliferation activity against A549, MCF7, and PC3 cancer cell lines with $\mathrm{IC}_{50}$ values of 27.09, 15.21, and $6.46 \mu \mathrm{M}$, respectively. 335 possessed anti-proliferation activity against A549, MCF7, and PC3 cancer cell lines with $\mathrm{IC}_{50}$ values of $2.58,42.45$, and $60.00 \mu \mathrm{M}$, respectively. 337 exerted anti-proliferation activity against A549, MCF7, and PC3 cancer cell lines with $\mathrm{IC}_{50}$ values of $37.93,56.06$, and $42.49 \mu \mathrm{M}$, respectively. $\mathbf{3 3 7}$ and $\mathbf{3 3 8}$ showed low anti-proliferation activity against the $\mathrm{A} 549$ cancer cell line with $\mathrm{IC}_{50}$ of 46.49 and $36.65 \mu \mathrm{M}$, respectively [87]. Cladiella sp. from Penghu Archipelago contained three new cembranoids (341, 342 and 344) which possessed anti-cancer activity. 341 and 344 exhibited moderate anti-proliferation activity toward the leukemia $\mathrm{K} 562$ cells with $\mathrm{IC}_{50}$ of 12.76 and $11.39 \mu \mathrm{g} / \mathrm{mL}$, respectively while 342 showed moderate anti-proliferation activity toward the leukemia MOLT-4 cells with $\mathrm{IC}_{50}$ of $18.83 \mu \mathrm{g} / \mathrm{mL}[88]$.

\subsection{Anti-inflammation}

Two novel compounds isolated from Sarcophyton elegans, 18 and 19, showed anti-inflammatory activity by inhibition of lipopolysaccharide (LPS)-induced nitrite oxide (NO) production by RAW 264.7 macrophages with $\mathrm{IC}_{50}$ values of 
18.2 and $32.5 \mu \mathrm{M}$, respectively [24]. Compound $\mathbf{3 1}$ isolated from Sarcophyton glaucom had inhibition activity towards the expression of inducible nitrite oxide synthase (iNOS) at 50 and $100 \mu \mathrm{M}$. This compound also showed activity against the expression of cyclooxygenase-2 (COX-2) at 25, 50, and $100 \mu \mathrm{M}$ in RAW 264.7 [33]. Other anti-inflammatory activities were also reported from a new compound, 57, and a known compound, 62. These two compounds showed inhibitory activity towards Tumor Necrosis Factor $\alpha$ (TNF$\alpha$ )-induced nuclear factor kappa B (NF- $\mathrm{kB}$ ) activation (a therapeutical target in cancer), with $\mathrm{IC}_{50}$ values of 35.23 and $22.52 \mu \mathrm{mol} / \mathrm{mL}$, respectively [31].

Novel compounds 69-73, and known compound 75, isolated from Sarcophyton cherbonnieri exhibited anti-inflammatory activity by the inhibition of $\mathrm{N}$-formylmethionineleucyl-phenylalanine/cytochalasin B (fMLF/CB)-induced superoxide anion generation and estalase release in human neutrophils at various potentials. Moderate inhibition activities were shown by $\mathbf{6 9 , 7 1}$ and $\mathbf{7 4}$ with respective values of $32.1,44.5$, and $64.6 \%$ superoxide anion generation, and 37.6, 35.6 , and $42.6 \%$ elastase release at $30 \mu \mathrm{M}$ were reported. Weaker activities were exerted by $\mathbf{7 0 , 7 2 , 7 3}$ and $\mathbf{7 5}$ with inhibitory effects of $4.0,6.4,2.6$, and $3.5 \%$ on superoxide anion generation, and inhibition by $23.5,27.6,30.5$, and $20.7 \%$ on elastase release have been reported [34]. Three known compounds $\mathbf{9 7 , 9 8}$, and $\mathbf{1 0 2}$, as well as the newly discovered compound, 100, isolated from Sarcophyton ehrenbergi, exerted anti-inflammatory activity by TNF- $\alpha$ secretion inhibition in RAW 264.7. The most potent activity was exhibited by $\mathbf{9 8}$ with $\mathrm{IC}_{50}$ similar to dexamethasone as the positive control ( $8.5 \mu \mathrm{M}$ vs. $8.7 \mu \mathrm{M}$, respectively). Meanwhile, the other three had moderate effects, with $\mathrm{IC}_{50}$ values of 28.5, 24.2, and 27.3 $\mu \mathrm{M}$ [39]. Other studies also reported several new and known compounds with similar activity. The $\mathrm{IC}_{50}$ of the three new compounds 129, 130, and 133 were $21.3,30.8$, and $38.6 \mu \mathrm{M}$, respectively, while those of the five known compounds 134-138 were 9.1, 15.4, 29.5, 12.5 , and $7.2 \mu \mathrm{M}$, respectively [43].

Compounds isolated from the soft coral Sinularia erecta, 170 and 171, exhibited anti-inflammatory activity through the inhibition of superoxide generation and elastase release in fMLP/CB-induced human neutrophils, with $\mathrm{IC}_{50}$ values of 2.3 and $8.5 \mu \mathrm{M}$, respectively [49]. Taiwanese Sinularia nanolobata contained four new cembranoids, 174-177. Only 177 showed anti-inflammatory activity in RAW 264.7 cells induced by LPS and it effectively reduced the levels of NO to $2.3 \%$ at a concentration of $100 \mu \mathrm{M}$. Moreover, 177 at a concentration of $50 \mu \mathrm{M}$ also exhibited good inhibitory activity against iNOS compared to the positive control aminoguanidine (AG). The level of NO was also reduced significantly to $19.6 \%$ while giving a $104.6 \%$ retention of cell viability [51]. The Bornean soft coral Sinularia sp. contained 182 and 183 which showed anti-inflammatory activity through inhibition of $\mathrm{NO}$, prostaglandin $\mathrm{E}_{2}\left(\mathrm{PGE}_{2}\right)$, Interleukin (IL)-1 $\beta$, IL-6, and iNOS in LPS-induced RAW 264.7 macrophages. Compounds 182 and 183 showed the most potent activity on the inhibition of NO production at 12.5 and $25.0 \mu \mathrm{g} / \mathrm{mL}$ compared to that of the negative control. The inhibition against $\mathrm{PGE}_{2}$ in LPS-induced RAW 264.7 macrophages of 182 and 183 were shown in a dose-dependent manner. Both compounds also showed significant inhibition against the accumulation of interleukin (IL-1 $\beta$ and IL-6) production at $25.0 \mu \mathrm{g} / \mathrm{mL}$, with a reduction of less than $10 \%$ to both interleukins. The inhibition of NO, IL-1 $\beta$, and IL-6 shown by $\mathbf{1 8 2}$ and $\mathbf{1 8 3}$ through the downregulation of iNOS expression. Weak inhibition was displayed against $\mathrm{PGE}_{2}$ by slight suppression of $\mathrm{COX}-2$ expression [56]. Compound $\mathbf{1 8 8}$ isolated from species collected in Hainan, China, showed high anti-inflammatory activity through inhibition of TNF- $\alpha$, with an $\mathrm{IC}_{50}$ of $2.7 \mu \mathrm{M}$ [58].

Among several compounds isolated from Sinularia flexibilis collected in Liuqiu, only compound $\mathbf{1 8 9}$ showed anti-inflammatory properties by significantly inhibiting the release of superoxide anion generation and elastase with $\mathrm{IC}_{50}$ values of 10.8 and $11.0 \mu \mathrm{M}$, respectively [94]. Seven of eight cembranoids successfully isolated from S. flexibilis $\mathbf{( 1 8 8 ,}$ $190,195,196,197,198$, and 199) showed anti-inflammatory activity through the inhibition of TNF- $\alpha$, with $\mathrm{IC}_{50}$ values of $2.7,4.7,20.7,38.9,>50,13.3$, and $4.2 \mu \mathrm{M}$, respectively [58].

Hainan soft coral Lobophytum crassum contained 13 cembranoids (224-236), five of which $(\mathbf{2 2 4}, \mathbf{2 3 0}, \mathbf{2 3 4}, \mathbf{2 3 5}$, 236) showed moderate anti-inflammatory activity through inhibition against LPS-induced $\mathrm{NO}$ production, with $\mathrm{IC}_{50}$ values of $17,13,24,8$, and $12 \mu \mathrm{M}$, respectively [64]. The Okinawan soft corals Lobophytum sp. were found to contain the cembranoids 240, 241 and $\mathbf{2 4 2}$ that exhibited antiinflammatory activity through reducing NO production, with $\mathrm{IC}_{50}$ values of $41.21,64.96$, and $74.76 \mu \mathrm{M}$, respectively [66]. Lai et al. [11] isolated $\mathbf{2 4 5}$ to $\mathbf{2 4 9}$ from Lobophytum crassum, which showed potent anti-inflammatory activity through inhibition of LPS induced IL-12 release by dendritic cells (DC), with inhibition potency of 93.4, 93.6, $86.3,77.0$ and $86.4 \%$, respectively. At the same time, inhibition of LPS induced NO release by DC of these five compounds (245-249) were recorded at values of $93.5 \%$ with DC survival at $76.0 \%, 95.9 \%$ with DC survival at $52.0 \%$, $86.1 \%$ with DC survival at $75.0 \%, 54.9 \%$ with DC survival at $85.0 \%$, and $86.1 \%$ with DC survival at $85.0 \%$ [11]. Cembranoids 262, 263 and 264 from the Okinawan soft coral Lobophytum sp. displayed anti-inflammatory effects through the suppression of NO production in a dose-dependent manner with $\mathrm{IC}_{50}$ of $10.67,13.92$, and $14.02 \mu \mathrm{M}$, respectively after $24 \mathrm{~h}$ in LPS-stimulated RAW 264.7 macrophage cells, at non-cytotoxic concentrations [69]. 
Two new compounds isolated from Briareum sp. (279 and 283) displayed anti-inflammatory activity by reducing iNOS level to $47.2 \%$ and $55.7 \%$, respectively, at a concentration of $10 \mu \mathrm{M}$ [74]. From a collection of Hainan Junceella fragilis, five cembranoids $(\mathbf{2 8 7}, \mathbf{2 8 8}, \mathbf{2 8 9}, \mathbf{2 9 0}, \mathbf{2 9 1})$ were isolated that exerted anti-inflammatory activity through the inhibition of NO production by 39.4, 42.7 (288 and $\mathbf{2 8 9}$ were tested together) and 36.3\% (290 and 291 were tested together), respectively (at $50 \mu \mathrm{M}$ ) in RAW 264.7 cells [76]. Ten cembranoids (292-301) isolated in 2017 from Klyxum flaccidum, of which 8 (292, 294-297, 299, 300, 301) possessed various anti-inflammatory activities. 292, 294, 299, 301 showed weak NO inhibitory activity with 25, 12, 20, 15\% inhibition, respectively, while 295 exerted moderate NO inhibition up to $65 \%$ ( $\mathrm{IC}_{50}$ of $46.7 \mu \mathrm{g} / \mathrm{mL}$ ) and 297 up to $64 \%\left(\mathrm{IC}_{50}\right.$ value of $\left.47.0 \mu \mathrm{g} / \mathrm{mL}\right)$. Furthermore, 296 and $\mathbf{3 0 0}$ strongly inhibited $88 \%$ and $87 \%$ of NO production at $50 \mu \mathrm{g} /$ $\mathrm{ml}$, respectively [77].

A novel cembranoid from Chicoreus ramosus, 302, showed anti-inflammatory activity through the inhibition of 5-lipooxygenase, with $\mathrm{IC}_{50}$ of $0.76 \mathrm{mg} / \mathrm{mL}$ [78]. Antiinflammatory activity was evident in $\mathbf{3 2 3}$ isolated from $K$. flaccidum, predicted to occur by a reduction in the level of elastase release to $59.66 \%$, with $\mathrm{IC}_{50}$ of $7.22 \mu \mathrm{M}$ at a concentration of $10 \mu \mathrm{M}$ relative to the control group [83]. Three out of four new cembranoids $(\mathbf{3 2 6}, \mathbf{3 2 8}, \mathbf{3 2 9})$ isolated from cultured type Briareum violaceum possessed antiinflammatory activity in LPS-induced RAW 264.7 macrophage cells by significantly inhibiting the expression of iNOS protein to $43,61,46 \%$, respectively [85]. Four new compounds (341-344) isolated in 2019 displayed various anti-inflammatory activities. Compounds 341 and 343 decreased the release of elastase with inhibition rates of $12.01 \%$ and $11.35 \%$, respectively, while 342 decreased the generation of superoxide anions by human neutrophils with the inhibition rate of $13.43 \%$, and $\mathbf{3 4 4}$ had an inhibition rate of $28.12 \%$. Additionally, $\mathbf{3 4 4}$ also decreased the release of elastase with the inhibition rate of $16.37 \%$ [88]. Three new cembranoids (346-348) isolated from aquacultured $B$. violaceum possessed anti-inflammatory activity by suppressing the release of inducible nitric oxide synthase (iNOS) in LPS-stimulated RAW 264.7 cells with values of $67.7,79.5$, and $61.9 \%$, respectively, compared to the results of the cells stimulated with only LPS at a concentration of $10 \mu \mathrm{M}$ [89]. Anti-inflammatory activity was also shown by the Arabian soft coral Stomopneustes variolaris, which produced the novel compound $\mathbf{3 5 2}$ that inhibited 5-lipoxygenase with $\mathrm{IC}_{50}$ of $2.01 \mathrm{mM}$, as compared to positive control ibuprofen $\left(\mathrm{IC}_{50} 4.50 \mathrm{mM}\right)$. The selectivity ratio of cyclooxygenase-1 $(\mathrm{COX}-1)$ to $\mathrm{COX}-2$ for the studied compound was found to be greater (1.25) than that of ibuprofen (0.43) [91]. Two known compounds isolated from Briareum excavatum, 353 and 354, displayed an anti-inflammatory effect, where $\mathbf{3 5 3}$ significantly reduced the release of COX-2 to $65.30 \%$ at $10 \mu \mathrm{M}$ in RAW 264.7 macrophages stimulated by LPS. In comparison, $\mathbf{3 5 4}$ showed anti-inflammatory activity through significantly reducing the release of iNOS to $60.29 \%$ at $10 \mu \mathrm{M}$ using the same model [92].

Known cembranoid 145 and 147 isolated from Sarcophyton digitatum showed anti-inflammatory activity through inhibiting the production of IL- $1 \beta$ to $68 \pm 1$ and $56 \pm 1 \%$, respectively in LPS-stimulated murine macrophages J774A. 1 at a concentration of $10 \mu \mathrm{g} / \mathrm{mL}$ with $\mathrm{IC}_{50}$ values of $10.7 \pm 2.7$ and $14.9 \pm 5.1 \mu \mathrm{g} / \mathrm{mL}$.[45]. In addition, Sarcophyton tenuispiculatum contained $\mathbf{1 5 6}$ which possessed anti-inflammatory activity through inhibiting the production of IL-1 $\beta$ to $56 \pm 1 \%$ in LPS-stimulated murine macrophage J774A. 1 cell at a concentration of $30 \mu \mathrm{m}$ [46]. New briaranes $\mathbf{3 5 7}$ and $\mathbf{3 6 0}$ exhibited anti-inflammatory activity by enhancing the release of iNOS (142.03 and $134.11 \%$, respectively) and COX-2 (159.21 and 196.03\%, respectively) in LPSstimulated RAW 264.7 macrophage cells at concentration of $10 \mu \mathrm{M}$ [93]. Sarcophyton roseum collected from Egypt contained 158 which possessed anti-inflammatory activity via iNOS inhibition with $\mathrm{IC}_{50}$ of $50 \mu \mathrm{M}$. Whilst, from the same species, 161 was isolated and showed anti-inflammatory activity via Nrf-2 induction at $100 \mu \mathrm{M}$ (2.1-fold), $50 \mu \mathrm{M}$ (1.4-fold), and $25 \mu \mathrm{M}$ (0.9-fold). Furthermore, 162 exhibited anti-inflammatory activity via iNOS inhibition with $\mathrm{IC}_{50}$ of $39 \mu \mathrm{M}$ and Nrf-2 induction at $100 \mu \mathrm{M}$ (1.8-fold), $50 \mu \mathrm{M}$ (1.5-fold), and $25 \mu \mathrm{M}$ (1.5-fold) [47].

Sarcophyton cherbonnieri contained cembranoids which possessed anti-inflammatory activity namely 163-169. Compound 163-169 showed inhibition on superoxide anion generation to $11.0 \pm 8.7 ; 29.8 \pm 9.8 ; 44.5 \pm 7.9 ; 6.4 \pm 7.3$; $6.2 \pm 5.5 ; 12.9 \pm 11.4$; and $17.1 \pm 11.6 \%$, respectively, at concentration of $30 \mu \mathrm{M}$. Furthermore, those compounds also inhibited the release of elastase to $35.1 \pm 10.6 ; 48.2 \pm 12.5$; $35.6 \pm 10.7 ; 27.6 \pm 12.8 ; 29.7 \pm 11.1 ; 16.7 \pm 10.2$; and $27.6 \pm 12.0 \%$, respectively, at concentration of $30 \mu \mathrm{M}$ [48]. Lastly, diterpenoid 222 isolated from Sinularia humilis collected in Ximao Islands have significant anti-inflammatory effects in LPS-stimulated BV-2 microglial cells with $83.96 \% \pm 2.02 \%$ and $65.70 \% \pm 2.76 \%$ NO level decrease at 10 and $20 \mu \mathrm{M}$, respectively [62].

\subsection{Other Biological Activities}

Other reported biological activities of cembranoids include induction of $\mathrm{T}$ lymphocyte proliferation. Three new compounds and a known compound isolated from Sarcophyton trocheliophorum, 86-89, were reported to be active on $\mathrm{T}$ lymphocyte cells from mice splenocytes. Compounds $\mathbf{8 6}$, $\mathbf{8 8}$, and 89 significantly induced cluster of differentiation 3 $\left(\mathrm{CD}^{+}\right) \mathrm{T}$ lymphocyte cells proliferation at $3 \mu \mathrm{M}$. In addition, 86 increased the $\mathrm{CD} 4^{+} / \mathrm{CD}^{+} \mathrm{T}$ lymphocyte cells ratio 
on mice splenocytes. In contrast, compound 87 exhibited decreased the $\mathrm{CD} 4^{+} / \mathrm{CD}^{+}$ratio [38]. Other active agents that exhibited activities related to $\mathrm{T}$ lymphocyte cell proliferation were two new compounds, 118 and 119, and also a known compound, 122, which were obtained from Sarcophyton mililatensis. Those compounds showed antiproliferation activity against Concanavalin A (ConA)induced $\mathrm{T}$ lymphocyte cell proliferation with $\mathrm{IC}_{50}$ values of $49.8,38.9$, and $11.4 \mu \mathrm{M}$, respectively. Additionally, the three compounds also exerted anti-proliferation activity on LPS-induced B lymphocyte cells, with $\mathrm{IC}_{50}$ values of 20.2, 22.1 and $4.9 \mu \mathrm{M}$, respectively. In the same report, a known compound, 121, also exhibited anti-proliferation activity on LPS-induced B lymphocyte cell proliferation, with $\mathrm{IC}_{50}$ of $4.8 \mu \mathrm{M}[42]$.

One study reported that two compounds, 81 (a new compound) and $\mathbf{8 4}$ (a known compound), extracted from Sarcophyton trocheliophorum showed inhibitory effect towards protein-tyrosine phosphatase 1B (PTP1B), with $\mathrm{IC}_{50}$ values of 19.9 and $15.4 \mu \mathrm{M}$, respectively [36]. This inhibitory effect is one of interest in the development of type 2 diabetes mellitus treatment as PTP1B is known as a negative regulator of the insulin signaling pathway [95]. Two new compounds, 107 and 110, isolated from Sarcophyton glaucum exhibited anti-larval settlement activity with an adhesive rate of 6.52 and $4.60 \%$ at $25 \mathrm{ppm}$, respectively. In the same study, three other known compounds, 115, 116 and 117, were shown to have anti-fouling activity against Balanus amphitrite, with adhesive rates of $8.19,14.14$, and $7.78 \%$ at $25 \mathrm{ppm}$, respectively [41]. One of the known compounds from Sarcophyton glaucum, 139, possessed neurological activity by competitive inhibition of neuronal glycine with inhibitory constant $\left(\mathrm{K}_{\mathrm{I}}\right)=109 \mu \mathrm{M}$. It did not have any effect on strychnine toxicity in a mouse experiment model [44].

Compounds 179 and 180 exhibited lethality against brine shrimp Artemia salina with lethal ratios of 90.5 and $90.0 \%$, respectively at a concentration of $50 \mu \mathrm{g} / \mathrm{mL}$ [53]. Several of the ten cembranoids $(\mathbf{1 7 7}, \mathbf{1 9 0}, \mathbf{2 0 4 - 2 1 1})$ isolated in 2019 were found to possess immunosuppressive activity. Cembranoid 160 showed significant inhibitory effects on the proliferation of LPS induced B lymphocyte cells, with an $\mathrm{IC}_{50}$ value of $9.2 \mu \mathrm{M}$. 177, 205, 207, 208, 209, 211 possessed immunosuppressive activities through potent inhibition on the proliferation of Con A-induced T lymphocyte cells, with $\mathrm{IC}_{50}$ values of $4.5,8.4,5.5,3.9,2.3$, and $6.1 \mu \mathrm{M}$, respectively. Compound 210 had considerable specific inhibition on B lymphocyte cell proliferation, with an $\mathrm{IC}_{50}$ value of $4.4 \mu \mathrm{M}$ and selectivity index (SI) of 10.9. This performance was much better than that of the positive control cyclosporin A (CsA) $(\mathrm{SI}=3.0) .210$ dose-dependently inhibited CD $19^{+}$ B lymphocyte cells proliferation by LPS induction, while it also showed modulatory effects on cytokine production, with the manifestation of decreased IL-6 production and slightly increased IL-10 production. $\mathbf{2 1 0}$ could suppress the derivational expression of CD86 on CD19 ${ }^{+}$B lymphocyte cells upon LPS stimulation. In vitro, LPS addition led to B lymphocyte cell growth and plasma cell formation (from 2.31\% to $11.0 \%$ ) and compound $\mathbf{2 1 0}$ dose-dependently inhibited the plasma cell proliferation [52]. 193 and 194 isolated from Yongxing Island Sinularia sp. possessed anti-diabetic activity through mild inhibitory activity against PTP1B with $\mathrm{IC}_{50}$ values of 47.5 and $12.5 \mathrm{mM}$, respectively, measured against sodium orthovanadate as the positive control $\left(\mathrm{IC}_{50} 881 \mu \mathrm{M}\right)$ [54]. Cembranoids $\mathbf{2 0 0}$ and $\mathbf{2 0 2}$ isolated from Xisha Islands Sinularia sp. can inhibit Alzheimer's amyloid-beta $42\left(\mathrm{~A}_{42}\right)$ aggregation at a concentration of $10 \mu \mathrm{M}$, with inhibition of 20.6 and $37.2 \%$, respectively. This potency was comparable to that of the positive control curcumin (20.5\%) [60]. Cembranoid $\mathbf{2 2 3}$ showed significant toxicity against $A$. salina with an $\mathrm{LD}_{50}$ value of $25 \mu \mathrm{g} / \mathrm{mL}$ [63].

A new cembranoid, 302, isolated from Chicoreus ramosus possessed anti-oxidant activity through 2,2-diphenyl1-picrylhydrazyl (DPPH) and 2,2'-azino-bis(3-ethylbenzothiazoline-6-sulfonic acid) $\left(\mathrm{ABTS}^{+}\right)$scavenging activity, with $\mathrm{IC}_{50}$ values of 0.26 and $0.36 \mathrm{mg} / \mathrm{mL}$, respectively [78]. Fourteen cembranoids have been isolated from the Caribbean Sea Eunicea sp., with some of them possessing antidiabetic activity. 304, 312-315 improved INS-1 pancreatic $\beta$ cell proliferation with a ratio of $1.9,1.7,1.7,2.2,1.4$, and 1.1, respectively compared with control. In this regard, $\mathbf{3 0 3}$ and 305-311 have not been tested for biological activity [79]. The Bornean soft coral Nephthea sp. contained 316, which showed insecticidal activity through repellent activity against maize weevil Sitophilus zeamais (grains pest) at $25 \mu \mathrm{g} / \mathrm{cm}^{2}$ [80]. In 2019, Castellanos et al. isolated 319 from Pseudoplexaura porosa and $\mathbf{3 2 0}$ from Eunicea knighti, which possessed AChE inhibition activity with $\mathrm{IC}_{50}$ of 1.40 and $0.358 \mu \mathrm{M}$, respectively. These compounds have the potential to be developed for neurodegenerative disease treatment, e.g. Alzheimer's disease [82]. The known compound, 351, isolated from L. flava possessed immunosuppressive activity through inhibiting the proliferation of ConA-induced $\mathrm{T}$ lymphocyte cells and/or LPS-induced B lymphocyte cells in vitro, with $\mathrm{IC}_{50}$ of 10.7 and $38.6 \mu \mathrm{M}$, respectively [90]. Compound $\mathbf{3 5 2}$ isolated from $S$. variolaris possessed potent anti-oxidant activity through DPPH and $\mathrm{ABTS}^{+}$scavenging activity with $\mathrm{IC}_{50}$ values of 1.41 and $1.61 \mathrm{mM}$, respectively, which were greater than that of the standard agent $\alpha$-tocopherol $\left(\mathrm{IC}_{50}\right.$ of 1.51 and $1.70 \mathrm{mM}$, respectively) [91]. Cembrane-type diterpenoid 213 and $\mathbf{2 1 6}$ showed inhibitory effect toward $\alpha$-Glucosidase with $\mathrm{IC}_{50}$ value of $10.65 \pm 0.16$ and $30.31 \pm 1.22 \mu \mathrm{M}$, respectively [61]. Furthermore, Lobophytum sp. from Xisha Island contained seven compounds namely 270-276 which exhibited a weak inhibitory effect of XBP-Splicing on B16-F10 tumor cells at a concentration of $10 \mu \mathrm{M}$ [72]. 
Many compounds reviewed in this paper were found to have no biological activity of interest reported in the respectively published article, such as 174-176 isolated from $S$. nanolobata [51]. The same was true to 184-186 isolated from S. flexibilis [94]; 192 isolated from the South China Sea soft coral Sinularia sp. [54], 201 and 203 isolated from Xisha Islands Sinularia sp. [60], 204 and 206 isolated from Xigu Island S. scabra [52], and neither did for 278, 280, 281 and 282 isolated from the soft coral Briareum sp. did not possess any biological activity [74]. Cembranoids 225-229 and 231-233 isolated by Zhao et al. in 2016 did not exhibit the activity of interest [64]. The same goes for three new cembranoids isolated by Zhang et al. [49], namely 265, 266 and 267 [70] as well as two new cembranoids, 268 and 269 , isolated by $\mathrm{Li}$ et al. [43] from the Hainan soft coral Lobophytum sp. [71]. No biological activity was detected in another new compound, 322, from $K$. flaccidum [83], as was the case with a new cembranoid, 324, isolated from J. fragilis [84], and with 327 derived from aquacultured B. violaceum [85]. Similarly, no biological activities of interest were recorded in the original published papers of the three novel cembranoids (330, 331 and 332) isolated from J. fragilis [86], two new cembranoids (339 and $\mathbf{3 4 0}$ ) from E. caribaeorum [87], 345 from aquacultured $B$. violaceum [89], two novel cembranoids, 349 and 350, from L. flava that originated from Xisha Islands [90], as well as a novel compound, 355, isolated from the Taiwanese soft coral B. excavatum [92].

Several cembranoid compounds have been recently discovered and have not been thoroughly tested for their biological activities [27, 28, 39]. Rahelivao et al. [59], isolated a new compound, 173, from the Madagascar soft coral S. gravis, but no biological activity was reported [50]. Dongsha Atoll soft corals L. crassum contained three novel compounds, 237, 238 and 239, which have not yet been explored for their biological activities [65]. 250 isolated from aquacultured $L$. crassum did not possess any biological activity of interest, while $\mathbf{2 5 1}$ has not been thoroughly tested [67]. 286 isolated from the Bornean soft coral Nephthea sp. [75] and 298 extracted from Klyxum flaccidum [77] have not been tested yet. Lastly, five briaranes were isolated from Briareum stechei which cultured in the National Museum of Marine Biology and Aquarium, Pingtung, Taiwan. briarenols W-Z 356-359 were the new reported compound and solenolide A 360 was the only known compound being isolated. Compound 357 and 360 were the only compound which exhibited anti-inflammatory activity by enhancing the release of iNOS and COX-2 [93].

\section{Conclusions}

Soft corals or Alcyonacea are rich potential sources of uniques compounds, particularly cembranoid diterpenes. These compounds have been demonstrated to display a spectrum of pharmacological activities such as anti-tumor, antibacterial and anti-inflammatory. Discoveries are being reported continually in the literature for cembranoid compounds isolated from soft corals as technologies for chemical

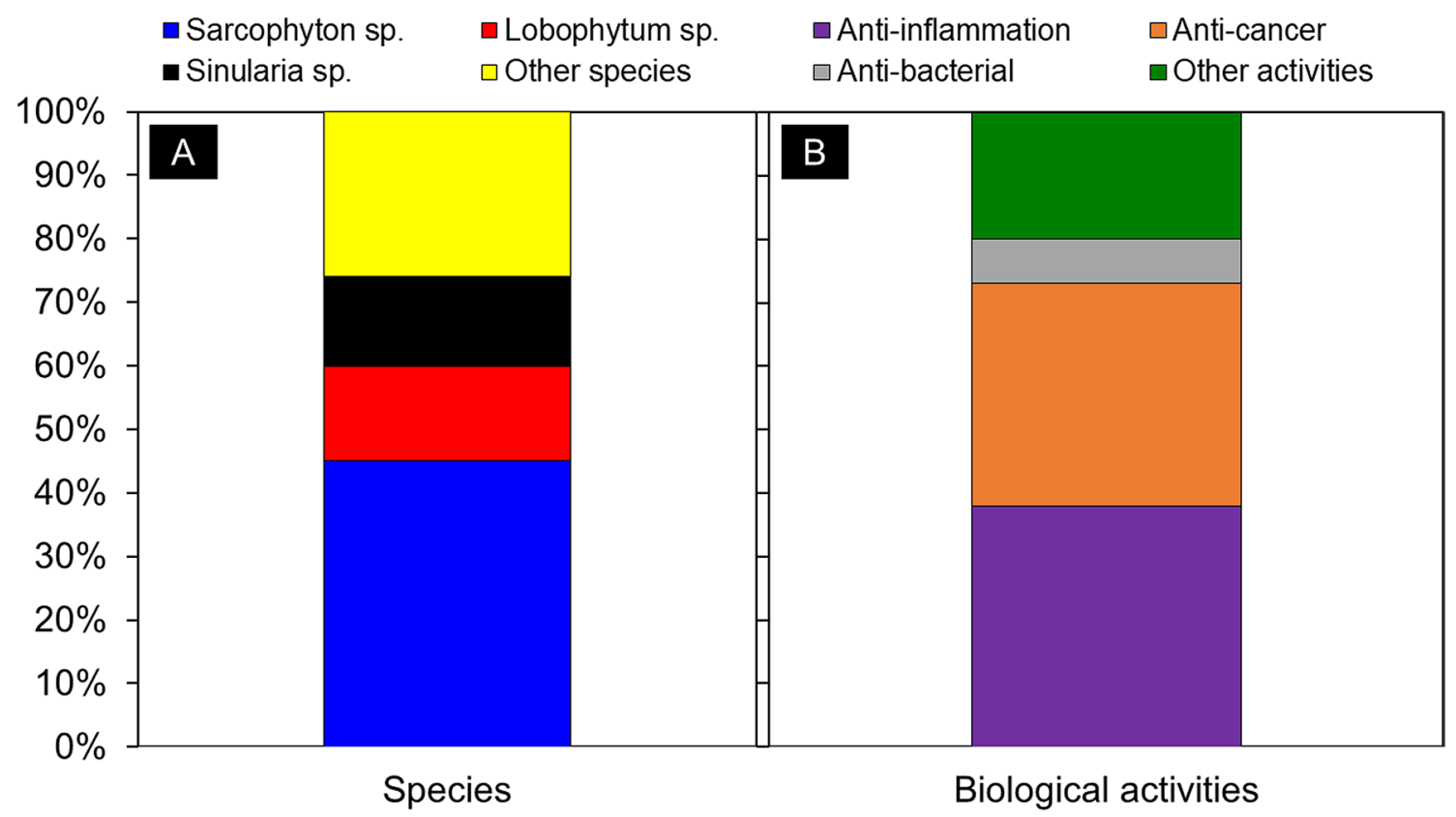

Fig. 12 The percentages of cembranoid-producing soft coral species (a). The percentage of different biological activities exhibited by cembranoids (b) 
extraction and characterization of secondary metabolites become more advanced.

This review provides an update on recent studies that encompass the isolation of up to 360 cembranoids from marine soft corals and brief accounts of their biological activities reported in the span of the recent five years. Most of the studied compounds were isolated from Sarcophyton sp. (45\%), followed by Lobophytum sp. (15\%), and Sinularia sp. (14\%). Other marine soft corals made up the remaining $26 \%$ of species. It is known that cembranoids from marine soft corals possess various biological characteristics. Antiinflammatory (38\%) was found to be the most common biological activity exhibited by cembranoids reported in this review, followed by anti-cancer (35\%), and anti-bacterial (7\%), whereas other activities encompassed the remaining $20 \%$ (Fig. 12). These early findings can lead to more detailed studies for marine cembranoid-based drug discovery and development.

Despite the abundance of unique cembranoids identified, the low quantitiy of isolated compounds may be a big challenge for drug applications' evaluation and development. We consider such approaches like synthesis and biosynthesis studies to be developed for applications of these cembranoids for drug discovery. Furthermore, with the recent advanced technology, various types of specific soft corals are becoming possible in aquaculture. This technology provides more abundant organisms to be extracted and a considerable quantity of molecules to be assessed for in vitro and in vivo study.

Acknowledgements This research was funded by the Indonesian Institute of Sciences (LIPI) through the DIPA 2021 Research Fund B-10405/IPH/HK.01.03/XI/2020. Authors greatly acknowledge to the Head of Research Center for Biotechnology, Indonesian Institute of Sciences, as well as the research and administration staff members for their support.

Author Contributions Resources, MYN, DS, AYN, SIR, AB, LS, AA, AP, FI, MFW; data curation, MYN and DS; writing-original draft preparation, MYN, DS, AYN, FI, AB, MYP; writing-review and editing, MYN, DS, MYP, AA, MF, FI, LS, and AP AB; visualization, AYN.MYP; supervision, MYP, AB; project administration, MYP, SIR, MFW, DAW, FI, AB; funding acquisition, MYP. All authors have read and agreed to the published version of the manuscript.

\section{Declarations}

Conflicts of interest The authors declare no conflict of interest.

Open Access This article is licensed under a Creative Commons Attribution 4.0 International License, which permits use, sharing, adaptation, distribution and reproduction in any medium or format, as long as you give appropriate credit to the original author(s) and the source, provide a link to the Creative Commons licence, and indicate if changes were made. The images or other third party material in this article are included in the article's Creative Commons licence, unless indicated otherwise in a credit line to the material. If material is not included in the article's Creative Commons licence and your intended use is not permitted by statutory regulation or exceeds the permitted use, you will need to obtain permission directly from the copyright holder. To view a copy of this licence, visit http://creativecommons.org/licenses/by/4.0/.

\section{References}

1. M. Townsend, K. Davies, N. Hanley, J.E. Hewitt, C.J. Lundquist, A.M. Lohrer, Front. Mar. Sci. 5, 359 (2018)

2. H. Malve, J. Pharm. Bioall. Sci. 8, 83 (2016)

3. E. O. Wilson and F. Peter M., Biodiversity (National Academies Press, Washington, D.C., 1988), p. 989.

4. D. Zeppilli, D. Leduc, C. Fontanier, D. Fontaneto, S. Fuchs, A.J. Gooday, A. Goineau, J. Ingels, V.N. Ivanenko, R.M. Kristensen, R.C. Neves, N. Sanchez, R. Sandulli, J. Sarrazin, M.V. Sørensen, A. Tasiemski, A. Vanreusel, M. Autret, L. Bourdonnay, M. Claireaux, V. Coquillé, L. De Wever, D. Rachel, J. Marchant, L. Toomey, D. Fernandes, Mar. Biodiv. 48, 35 (2018)

5. O.K. Radjasa, Y.M. Vaske, G. Navarro, H.C. Vervoort, K. Tenney, R.G. Linington, P. Crews, Bioorg. Med. Chem. 19, 6658 (2011)

6. S. Aratake, T. Tomura, S. Saitoh, R. Yokokura, Y. Kawanishi, R. Shinjo, J.D. Reimer, J. Tanaka, H. Maekawa, PLoS ONE 7, e30410 (2012)

7. Y. Li, G. Pattenden, Nat. Prod. Rep. 28, 1269 (2011)

8. M.T.R. Almeida, M.I.G. Moritz, K.C.C. Capel, C.D. Pérez, E.P. Schenkel, Rev. Bras 24, 446 (2014)

9. Y. González, D. Torres-Mendoza, G.E. Jones, P.L. Fernandez, Mediators Inflamm. 2015, 1 (2015)

10. E. Fattorusso, P. Luciano, M.Y. Putra, O. Taglialatela-Scafati, A. Ianaro, E. Panza, G. Bavestrello, C. Cerrano, Tetrahedron 67, $7983(2011)$

11. K.H. Lai, W.J. You, C.C. Lin, M. El-Shazly, Z.J. Liao, J.H. Su, Mar. Drugs 15, 327 (2017)

12. D. Rodríguez, Y. Li, H. Dhasmana, C.L. Barnes, J. Nat. Prod. 56, $1101(1993)$

13. M. Gill, W. Steglich, Fortschritte Der Chemie Organischer Naturstoffe/Progress in the Chemistry of Organic Natural Products (Springer, Vienna, 1987).

14. Z. Xi, W. Bie, W. Chen, D. Liu, L. Ofwegen, P. Proksch, W. Lin, Mar. Drugs 11, 3186 (2013)

15. B. Yang, X.-F. Zhou, X.-P. Lin, J. Liu, Y. Peng, X.-W. Yang, Y. Liu, Curr. Org. Chem. 16, 1512 (2012)

16. J.W. Blunt, B.R. Copp, M.H.G. Munro, P.T. Northcote, M.R. Prinsep, Nat. Prod. Rep. 28, 196 (2011)

17. T. Kamada, C.S. Phan, H.S. Tin, C.S. Vairappan, T.S.T. Muhammad, Nat. Prod. Commun. 11, 1077 (2016)

18. M.Y. Putra, A. Saparhadi, F. Karim, T. Murniasih, R.T. Swasono, Squalen Bull. Mar. Fish. Postharvest Biotechnol. 11, 53 (2016)

19. P. Sun, Q. Yu, J. Li, R. Riccio, G. Lauro, G. Bifulco, T. Kurtán, A. Mándi, H. Tang, T.J. Li, C.L. Zhuang, W.H. Gerwick, W. Zhang, J. Nat. Prod. 79, 2552 (2016)

20. W.T. Chen, L.F. Liang, X.W. Li, W. Xiao, Y.W. Guo, Nat. Prod. Bioprospect. 6, 97 (2016)

21. M. Zubair, W. Alarif, K. Al-Footy, M. Ph, M. Ali, S. Basaif, S. Al-Lihaibi, S.-E. Ayyad, Turk. J. Chem. 40, 385 (2016)

22. G.H. Tang, Z.H. Sun, Y.H. Zou, S. Yin, Molecules 21, 587 (2016)

23. M. Shaaban, M.A. Ghani, K.A. Shaaban, J. Chem. Sci. 71, 1211 (2016)

24. W. Li, Y.H. Zou, M.X. Ge, L.L. Lou, Y.S. Xu, A. Ahmed, Y.Y. Chen, J.S. Zhang, G.H. Tang, S. Yin, Mar. Drugs 15, 85 (2017)

25. M.E.F. Hegazy, A.I. Elshamy, T.A. Mohamed, A.R. Hamed, M.A.A. Ibrahim, S. Ohta, P.W. Paré, Mar. Drugs 15, 192 (2017) 
26. M. Shaaban, A.M. El-Hagrassi, M.A. Abdelghani, A.F. Osman, J. Biosci. 73, 325 (2017)

27. M.P. Rahelivao, T. Lübken, M. Gruner, O. Kataeva, R. Ralambondrahety, H. Andriamanantoanina, M.P. Checinski, I. Bauer, H.J. Knölker, Org. Biomol. Chem. 15, 2593 (2017)

28. L.F. Liang, W.T. Chen, X.W. Li, H.Y. Wang, Y.W. Guo, Sci. Rep. 7, 1 (2017)

29. H. Januar, N. Zamani, D. Soedharma, E. Chasanah, Pharmacognosy Research 9, 65 (2017)

30. L.F. Liang, W.T. Chen, E. Mollo, L.G. Yao, H.Y. Wang, W. Xiao, Y.W. Guo, Chem. Biodivers. 14, e1700079 (2017)

31. S. Li, F. Ye, Z. Zhu, H. Huang, S. Mao, Y. Guo, Acta Pharm. Sin. B 8, 944 (2018)

32. H. M. Hassan, M. E. Rateb, M. H. Hassan, A. M. Sayed, S. Shabana, M. Raslan, E. Amin, F. A. Behery, O. M. Ahmed, A. B. Muhsinah, T. A. M. Gulder, U. R. Abdelmohsen, Mar. Drugs 17, (2019).

33. Y.W. Ahmed, C.Y. Chen, Y.J. Huang, C.C. Tseng, C.F. Lin, YCWu Dai, J.H. Sheu, Mar. Drugs 16, 210 (2018)

34. C.C. Peng, C.Y. Huang, A.F. Ahmed, T.L. Hwang, C.F. Dai, J.H. Sheu, Mar. Drugs 16, 276 (2018)

35. M. Shaaban, M.Y. Issa, M.A. Ghani, A. Hamed, A.B. Abdelwahab, Nat. Prod. Res. 33, 24 (2019)

36. L.F. Liang, T. Kurtán, A. Mándi, L.G. Yao, J. Li, L.F. Lan, Y.W. Guo, Tetrahedron 74, 1933 (2018)

37. K. Tani, T. Kamada, C.-S. Phan, and C. S. Vairappan, Nat Product Commun. 14, 1934578X1986837 (2019).

38. P. Sun, F.Y. Cai, G. Lauro, H. Tang, L. Su, H.L. Wang, H.H. Li, A. Mándi, T. Kurtán, R. Riccio, G. Bifulco, W. Zhang, J. Nat. Prod. 82, 1264 (2019)

39. G. Li, H. Li, Q. Zhang, M. Yang, Y.C. Gu, L.F. Liang, W. Tang, Y.W. Guo, J. Org. Chem. 84, 5091 (2019)

40. M.E.F. Hegazy, T.A. Mohamed, A.I. Elshamy, A.R. Hamed, M.A.A. Ibrahim, S. Ohta, A. Umeyama, P.W. Paré, T. Efferth, RSC Adv. 9, 27183 (2019)

41. J. Zhang, X. Tang, X. Han, D. Feng, X. Luo, L. Van Ofwegen, P. Li, G. Li, Inorg. Chem. Front. 6, 2004 (2019)

42. M. Yang, X.L. Li, J.R. Wang, X. Lei, W. Tang, X.W. Li, H. Sun, Y.W. Guo, J. Org. Chem. 84, 2568 (2019)

43. G. Li, H. Li, W. Tang, L.G. Yao, L.F. Liang, Y.W. Guo, Bioorg. Chem. 101, 103993 (2020)

44. H.A. Saleh, K.M. Raafat, T.A. Temraz, N. Noureldin, H.G. Breitinger, U. Breitinger, Neurotoxicology 80, 105 (2020)

45. T.Y. Huang, C.Y. Huang, C.H. Chao, C.C. Lin, C.F. Dai, J.H. Su, P.J. Sung, S.H. Wu, J.H. Sheu, Mar. Drugs 18, 452 (2020)

46. T.Y. Huang, C.Y. Huang, S.R. Chen, J.R. Weng, T.H. Tu, Y.B. Cheng, S.H. Wu, J.H. Sheu, Mar. Drugs 19, 8 (2020)

47. M. M. A. Ahmed, M. A. Albadry, E. A. Ragab, E. M. El-Ghaly, S. K. Ismail, Z. Ali, S. I. Khan, A. G. Chittiboyina, and I. A. Khan, Nat. Product Res. 1 (2020).

48. C.C. Peng, C.Y. Huang, A.F. Ahmed, T.L. Hwang, J.H. Sheu, Mar. Drugs 18, 573 (2020)

49. C.Y. Huang, Y.J. Tseng, U. Chokkalingam, T.L. Hwang, C.H. Hsu, C.F. Dai, P.J. Sung, J.H. Sheu, J. Nat. Prod. 79, 1339 (2016)

50. M.P. Rahelivao, M. Gruner, T. Lübken, D. Islamov, O. Kataeva, H. Andriamanantoanina, I. Bauer, H.-J. Knölker, Org. Biomol. Chem. 14, 989 (2016)

51. C.H. Chao, C.Y. Wu, C.Y. Huang, H.C. Wang, C.F. Dai, Y.C. Wu, J.H. Sheu, Mar. Drugs 14, 150 (2016)

52. M. Yang, H. Li, Q. Zhang, Q.-H. Wu, G. Li, K.-X. Chen, Y.-W. Guo, W. Tang, X.-W. Li, Bioorg. Med. Chem. 27, 3469 (2019)

53. M. Wang, J. Jin, L. Li, F. Cao, C. Wang, C.-Y. Wang, Chem. Nat. Compd. 53, 181 (2017)

54. G.F. Qin, X.L. Tang, Y.T. Sun, X.C. Luo, J. Zhang, L. van Ofwegen, P.J. Sung, P.L. Li, G.Q. Li, Mar. Drugs 16, 127 (2018)
55. T.C. Tsai, K.H. Lai, J.H. Su, Y.J. Wu, J.H. Sheu, Mar. Drugs 16, 104 (2018)

56. T. Kamada, M.C. Kang, C.S. Phan, I. Zanil, Y.J. Jeon, C. Vairappan, Mar. Drugs 16, 99 (2018)

57. C.H. Wu, C.H. Chao, T.Z. Huang, C.Y. Huang, T.L. Hwang, C.F. Dai, J.H. Sheu, Mar. Drugs 16, 278 (2018)

58. Q. Wu, X.W. Li, H. Li, L.G. Yao, W. Tang, Z.H. Miao, H. Wang, Y.W. Guo, Bioorg. Med. Chem. Lett. 29, 185 (2019)

59. C.I. Yu, C.Y. Chen, W. Liu, P.C. Chang, C.W. Huang, K.F. Han, I.P. Lin, M.Y. Lin, C.H. Lee, Mar. Drugs 16, 387 (2018)

60. C.S. Jiang, T. Ru, L.G. Yao, Z.H. Miao, Y.W. Guo, Fitoterapia 136, $104176(2019)$

61. M.J. Wu, H. Wang, C.S. Jiang, Y.W. Guo, Bioorg. Chem. 104, 104281 (2020)

62. L.L. Sun, W.S. Li, J. Li, H.Y. Zhang, L.G. Yao, H. Luo, Y.W. Guo, X.W. Li, J. Org. Chem. 86, 3367 (2021)

63. K.O. Al-Footy, W.M. Alarif, M.S. Zubair, M.A. Ghandourah, M.M. Aly, Trop. J. Pharm. Res. 15, 1431 (2016)

64. M. Zhao, S. Cheng, W. Yuan, Y. Xi, X. Li, J. Dong, K. Huang, K. Gustafson, P. Yan, Mar. Drugs 14, 111 (2016)

65. T.A. Mohamed, A.I. Elshamy, T.A. Hussien, J.H. Su, J.H. Sheu, M.E.F. Hegazy, J. Asian Nat. Prod. Res. 19, 201 (2017)

66. P. Roy, R. Ashimine, H. Miyazato, J. Taira, K. Ueda, Molecules 21, 679 (2016)

67. B. R. Peng, M. C. Lu, M. El-Shazly, S. L. Wu, K. H. Lai, and J. H. Su, Marine Drugs 16, (2018).

68. Y.C. Liu, B.R. Peng, K.C. Hsu, M. El-Shazly, S.P. Shih, T.E. Lin, F.W. Kuo, Y.C. Chou, H.Y. Lin, M.C. Lu, Mar. Drugs 18, 382 (2020)

69. P.K. Roy, S. Roy, K. Ueda, Fitoterapia 136, 104162 (2019)

70. Q. Zhang, X.-W. Li, L.-G. Yao, B. Wu, Y.-W. Guo, Fitoterapia 133, 70 (2019)

71. S.W. Li, C. Cuadrado, L.G. Yao, A.H. Daranas, Y.W. Guo, Org. Lett. 22, 4093 (2020)

72. S.W. Li, C. Cuadrado, X.J. Huan, L.G. Yao, Z.H. Miao, A. Hernandez Daranas, Y.W. Guo, Bioorgan Chem. 103, 104223 (2020)

73. K.Y. Peng, N.F. Chen, Z.C. Chen, K.H. Tsui, Z.H. Wen, Y. Di Su, Y.C. Chang, Y.H. Chen, M.C. Lu, L.S. Fang, J.J. Chen, T.Y. Wu, Y.C. Wu, P.J. Sung, Tetrahedron Lett. 57, 4239 (2016)

74. Y.D. Su, C.S. Sung, Z.H. Wen, Y.H. Chen, Y.C. Chang, J.J. Chen, L.S. Fang, Y.C. Wu, J.H. Sheu, P.J. Sung, Int. J. Mol. Sci. 17, 79 (2016)

75. T. Ishii, T. Kamada, C.S. Vairappan, J. Asian Nat. Prod. Res. 18 415 (2016)

76. W. Cheng, X. Li, F. Yin, L. van Ofwegen, W. Lin, Chem. Biodivers. 14, e1700053 (2017)

77. C.R.T. Ahmed, C.Y. Huang, S.Y. Wang, J.H. Sheu, Mar. Drugs 15, 23 (2017)

78. K. Chakraborty, S. Salas, M. Joy, Nat. Prod. Res. 34, 1678 (2020)

79. G. Porras, A.R. Díaz-Marrero, J.M. de la Rosa, L. D'Croz, N. de Pablo, G. Perdomo, I. Cózar-Castellano, J. Darias, M. Cueto, Tetrahedron 74, 2056 (2018)

80. T. Ishii, T. Kamada, C.-S. Phan, C.S. Vairappan, Sains Malays. 47, 319 (2018)

81. K. Bauer, M. Puyana, L. Castellanos, E. Tello, Rec. Nat. Prod. 13, 243 (2019)

82. F. Castellanos, F. Amaya-García, E. Tello, F.A. Ramos, A. Umaña, M. Puyana, J.A.L.C. Resende, L. Castellanos, Nat. Prod. Res. 33, $3533(2019)$

83. A. Tseng, T. Huang, O. Tai, W. Hwang, S. Dai, Mar. Drugs 17, 461 (2019)

84. N. N. Thi, H. Tran, T. Hong, T. Nguyen Van, C. N. Xuan, N. N. Hoai, T. Do Cong, K. Phan Van, and M. Chau Van, Nat Product Res 34, 385 (2020). 
85. P.C. Huang, C.C. Tseng, B.R. Peng, C.C. Hu, N.C. Lin, N.F. Chen, J.J. Chen, Z.H. Wen, Y.C. Wu, P.J. Sung, Tetrahedron 75, 921 (2019)

86. P.J. Sung, C.Y. Lee, C.C. Lin, W.F. Chen, G.H. Lee, Z.H. Wen, L.S. Fang, Y.H. Kuo, Heterocycles 98, 984 (2019)

87. S.L. Molina, A.M. Forero, F.I. Ayala, M. Puyana, S. Zea, L. Castellanos, D. Muñoz, G. Arboleda, A.G. Sandoval-Hernández, F.A. Ramos, Mar. Drugs 18, 4 (2019)

88. Y.F. Chen, W.F. Chen, Z.H. Wen, T.L. Hwang, Z.J. Zhang, P.J. Sung, Phytochem. Lett. 33, 31 (2019)

89. P.C. Huang, W.S. Lin, B.R. Peng, Y.C. Chang, L.S. Fang, G.Q. Li, T.L. Hwang, Z.H. Wen, P.J. Sung, Mar. Drugs 17, 214 (2019)
90. Q. Wu, H. Li, M. Yang, A.Q. Jia, W. Tang, H. Wang, Y.W. Guo, Fitoterapia 134, 481 (2019)

91. P. Francis, K. Chakraborty, Med. Chem. Res. 29, 656 (2020)

92. T.H. Huynh, Y.M. Chang, S.N. Yang, G.H. Lee, Z.H. Wen, Y.J. Wu, T.R. Su, P.J. Sung, J. Mol. Struct. 1223, 128970 (2021)

93. Y.Y. Chen, Y.L. Zhang, G.H. Lee, L.K. Tsou, M.M. Zhang, H.P. Hsieh, J.J. Chen, C.Y. Ko, Z.H. Wen, P.J. Sung, Mar. Drugs 19, $77(2021)$

94. C. H. Wu, C. H. Chao, T. Z. Huang, C. Y. Huang, T. L. Hwang, C. F. Dai, and J. H. Sheu, Marine Drugs 16, (2018).

95. P. Combs, J. Med. Chem. 53, 2333 (2010) 